\title{
The hyperbolic Anderson model: moment estimates of the Malliavin derivatives and applications
}

\author{
Raluca M. Balan ${ }^{1}$ - David Nualart ${ }^{2}$ - Lluís Quer-Sardanyons ${ }^{3}$. \\ Guangqu Zheng ${ }^{4}$ (D)
}

This article is dedicated to István Gyöngy on the occasion of his 70th birthday.

Received: 23 September 2021 / Revised: 27 October 2021 / Accepted: 9 November 2021 /

Published online: 18 January 2022

(c) The Author(s) 2022

\begin{abstract}
In this article, we study the hyperbolic Anderson model driven by a space-time colored Gaussian homogeneous noise with spatial dimension $d=1,2$. Under mild assumptions, we provide $L^{p}$-estimates of the iterated Malliavin derivative of the solution in terms of the fundamental solution of the wave solution. To achieve this goal, we rely heavily on the Wiener chaos expansion of the solution. Our first application are quantitative central limit theorems for spatial averages of the solution to the hyperbolic Anderson model, where the rates of convergence are described by the total variation distance. These quantitative results have been elusive so far due to the temporal correlation of the noise blocking us from using the Itô calculus. A novel ingredient to overcome this difficulty is the second-order Gaussian Poincaré inequality coupled with the application of the aforementioned $L^{p}$-estimates of the first two Malliavin derivatives. Besides, we provide the corresponding functional central limit theorems. As a second application, we establish the absolute continuity of the law for the hyperbolic Anderson model. The $L^{p}$-estimates of Malliavin derivatives are crucial ingredients to verify a local version of Bouleau-Hirsch criterion for absolute continuity. Our approach substantially simplifies the arguments for the one-dimensional case, which has been studied in the recent work by [2].
\end{abstract}

Research supported by a grant from the Natural Sciences and Engineering Research Council of Canada. Supported by NSF Grant DMS 1811181.

Supported by the Grant PGC2018-097848-B-I00 (Ministerio de Economía y Competitividad).

Guangqu Zheng

zhengguangqu@gmail.com

Extended author information available on the last page of the article 
Keywords Hyperbolic Anderson model · Wiener chaos expansion · Malliavin calculus · Second-order Poincaré inequality · Quantitative central limit theorem · Riesz kernel · Dalang's condition

Mathematics Subject Classification 60H15 - 60H07 · 60G15 · 60F05

\section{Introduction}

One of the main tools of modern stochastic analysis is Malliavin calculus. To put it short, this is a differential calculus on a Gaussian space that represents an infinite dimensional generalization of the usual analytical concepts on an Euclidean space. The Malliavin calculus (also known as the stochastic calculus of variations) was initiated by Paul Malliavin [21] to give a probabilistic proof of Hörmander's "sum of squares" theorem. It has been further developed by Stroock, Bismut, Watanabe and others. One of the main applications of Malliavin calculus is the study of regularity properties of probability laws, for example, the laws of the solutions to certain stochastic differential equations and stochastic partial differential equations (SPDEs), see e.g. [27, Chapter 2]. The Malliavin calculus is also useful in formulating and interpreting stochastic (partial) differential equations when the solution is not adapted to a Brownian filtration, which is the case of SPDEs driven by a Gaussian noise that is colored in time.

Recently, the Malliavin calculus has found another important application in the work of Nualart and Ortiz-Latorre [28], which paved the road for Stein to meet Malliavin. The authors of [28] applied the Malliavin calculus (notably the integration by parts formula) to characterize the convergence in law of a sequence of multiple Wiener integrals, and they were able to give new proofs for the fourth moment theorems of Nualart, Peccati and Tudor [30,37]. Soon after the work [28], Nourdin and Peccati combined Malliavin calculus and Stein's method of normal approximation to quantify the fourth moment theorem. Their work [24] marked the birth of the so-called Malliavin-Stein approach. This combination works admirably well, partially because one of the fundamental ingredients in Stein's method—-the so-called Stein's lemma (2.6)—-that characterizes the normal distribution, is nothing else but a particular case of the integration by parts formula (2.5) in Malliavin calculus. We refer interested readers to [44, Section 1.2] for a friendly introduction to this approach.

The central object of study in this paper is the stochastic wave equation with linear Gaussian multiplicative noise (in Skorokhod sense):

$$
\left\{\begin{array}{l}
\frac{\partial^{2} u}{\partial t^{2}}=\Delta u+u \dot{W} \\
u(0, x)=1, \quad \frac{\partial u}{\partial t}(0, x)=0
\end{array} \quad \text { on } \mathbb{R}_{+} \times \mathbb{R}^{d} \text { for } d \in\{1,2\}\right.
$$

where $\Delta$ is the Laplacian in space variables and the Gaussian noise $\dot{W}$ has the following correlation structure

$$
\mathbb{E}[\dot{W}(t, x) \dot{W}(s, y)]=\gamma_{0}(t-s) \gamma(x-y),
$$


with the following standing assumptions:

(i) $\gamma_{0}: \mathbb{R} \rightarrow[0, \infty]$ is locally integrable and non-negative definite;

(ii) $\gamma$ is a non-negative and non-negative definite measure on $\mathbb{R}^{d}$ whose spectral measure $\mu^{1}$ satisfies Dalang's condition:

$$
\int_{\mathbb{R}^{d}} \frac{1}{1+|\xi|^{2}} \mu(d \xi)<\infty
$$

where $|\xi|$ denotes the Euclidean norm of $\xi \in \mathbb{R}^{d}$.

An important example of the temporal correlation is the Riesz kernel $\gamma_{0}(t)=|t|^{-\alpha_{0}}$ for some $\alpha_{0} \in(0,1)$ (with $\gamma_{0}(0)=\infty$ ).

Equation (1.1) is also known in the literature as the hyperbolic Anderson model, by analogy with the parabolic Anderson model in which the wave operator is replaced by the heat operator. The noise $\dot{W}$ can be formally realized as an isonormal Gaussian process $W=\{W(\phi): \phi \in \mathcal{H}\}$ and here $\mathcal{H}$ is a Hilbert space that is the completion of the set $C_{c}^{\infty}\left(\mathbb{R}_{+} \times \mathbb{R}^{d}\right)$ of infinitely differentiable functions with compact support under the inner product

$$
\begin{aligned}
\langle\phi, \psi\rangle_{\mathcal{H}} & =\int_{\mathbb{R}_{+}^{2} \times \mathbb{R}^{2 d}} \phi(t, x) \psi(s, y) \gamma_{0}(t-s) \gamma(x-y) d t d x d s d y \\
& =\int_{\mathbb{R}_{+}^{2}} d t d s \gamma_{0}(t-s) \int_{\mathbb{R}^{d}} d x \phi(t, x)[\psi(s, \bullet) * \gamma](x),
\end{aligned}
$$

where we write $\gamma(x)$ for the density of $\gamma$ if it exists and we shall use the definition (1.4) instead of (1.3) when $\gamma$ is a measure. In (1.4), * denotes the convolution in the space variable and $\gamma_{0}(t)=\gamma_{0}(-t)$ for $t<0$. We denote by $\mathcal{H}^{\otimes p}$ the $p$ th tensor product of $\mathcal{H}$ for $p \in \mathbb{N}^{*}$, see Sect. 2 for more details.

As mentioned before, the existence of a temporal correlation $\gamma_{0}$ prevents us from defining equation (1.1) in the Itô sense due to a lack of the martingale structure. In the recent work [3] by Balan and Song, the following results are established using Malliavin calculus. Let $G_{t}$ denote the fundamental solution to the corresponding deterministic wave equation, that is, for $(t, z) \in(0, \infty) \times \mathbb{R}^{d}$,

$$
G_{t}(z):= \begin{cases}\frac{1}{2} \mathbf{1}_{\{|z|<t\}} & \text { if } d=1 ; \\ \frac{1}{2 \pi \sqrt{t^{2}-|z|^{2}}} \mathbf{1}_{\{|z|<t\}} & \text { if } d=2 .\end{cases}
$$

To ease the notation, we will stick to the convention that

$$
G_{t}(z)=0 \text { when } t \leq 0 \text {. }
$$

\footnotetext{
1 The spectral measure $\mu$ of $\gamma$ is a tempered measure on $\mathbb{R}^{d}$ such that $\gamma=\mathcal{F} \mu$, that is, $\gamma$ is the Fourier transform of $\mu$, and its existence is guaranteed by the Bochner-Schwarz theorem.
} 
Definition 1.1 Fix $d \in\{1,2\}$. We say that a square-integrable process $u=\{u(t, x)$ : $\left.(t, x) \in \mathbb{R}_{+} \times \mathbb{R}^{d}\right\}$ is a mild Skorokhod solution to the hyperbolic Anderson model (1.1) if $u$ has a jointly measurable modification (still denoted by $u$ ) such that $\sup \left\{\mathbb{E}\left[u(t, x)^{2}\right]:(t, x) \in[0, T] \times \mathbb{R}^{d}\right\}<\infty$ for any finite $T$; and for any $t>0$ and $x \in \mathbb{R}^{d}$, the following equality holds in $L^{2}(\Omega)$ :

$$
u(t, x)=1+\int_{0}^{t} \int_{\mathbb{R}^{d}} G_{t-s}(x-y) u(s, y) W(d s, d y),
$$

where the above stochastic integral is understood in the Skorokhod sense and the process $(s, y) \in \mathbb{R}_{+} \times \mathbb{R}^{d} \longmapsto \mathbf{1}_{(0, t)}(s) G_{t-s}(x-y) u(s, y)$ is Skorokhod integrable. See Definition 5.1 in [3] and Definition 1.1 in [2].

It has been proved in [3, Section 5] that equation (1.1) admits a unique mild Skorokhod solution $u$ with the following Wiener chaos expansion:

$$
u(t, x)=1+\sum_{n \geq 1} I_{n}\left(\tilde{f}_{t, x, n}\right)
$$

where $I_{n}$ denotes the $n$th multiple Wiener integral associated to the isonormal Gaussian process $W$ (see Sect. 2 for more details), $f_{t, x, n} \in \mathcal{H}^{\otimes n}$ is defined by (with the convention (1.6) in mind)

$$
f_{t, x, n}\left(t_{1}, x_{1}, \ldots, t_{n}, x_{n}\right):=G_{t-t_{1}}\left(x-x_{1}\right) G_{t_{1}-t_{2}}\left(x_{1}-x_{2}\right) \cdots G_{t_{n-1}-t_{n}}\left(x_{n-1}-x_{n}\right),
$$

and $\widetilde{f}_{t, x, n}$ is the canonical symmetrization of $f_{t, x, n} \in \mathcal{H}^{\otimes n}$ given by

$$
\widetilde{f}_{t, x, n}\left(t_{1}, x_{1}, \ldots, t_{n}, x_{n}\right):=\frac{1}{n !} \sum_{\sigma \in \mathfrak{S}_{n}} f_{t, x, n}\left(t_{\sigma(1)}, x_{\sigma(1)}, \ldots, t_{\sigma(n)}, x_{\sigma(n)}\right),
$$

where the sum in (1.9) runs over $\mathfrak{S}_{n}$, the set of permutations on $\{1,2, \ldots, n\}$. For example, $f_{t, x, 1}\left(t_{1}, x_{1}\right)=G_{t-t_{1}}\left(x-x_{1}\right)$ and

$$
\begin{aligned}
& \widetilde{f}_{t, x, 2}\left(t_{1}, x_{1}, t_{2}, x_{2}\right) \\
& \quad=\frac{1}{2}\left(G_{t-t_{1}}\left(x-x_{1}\right) G_{t_{1}-t_{2}}\left(x_{1}-x_{2}\right)+G_{t-t_{2}}\left(x-x_{2}\right) G_{t_{2}-t_{1}}\left(x_{2}-x_{1}\right)\right) .
\end{aligned}
$$

We would like to point out that in the presence of temporal correlation, there is no developed solution theory for the nonlinear wave equation (replacing $u \dot{W}$ in (1.1) by $\sigma(u) \dot{W}$ for some deterministic Lipschitz function $\sigma: \mathbb{R} \rightarrow \mathbb{R}$ ). We regard this as a totally different problem.

Now let us introduce the following hypothesis when $d=2$ :

(a) $\gamma \in L^{\ell}\left(\mathbb{R}^{2}\right)$ for some $\ell \in(1, \infty)$,

(b) $\gamma(x)=|x|^{-\beta}$ for some $\beta \in(0,2)$,

(c) $\gamma\left(x_{1}, x_{2}\right)=\gamma_{1}\left(x_{1}\right) \gamma_{2}\left(x_{2}\right)$, where $\gamma_{i}\left(x_{i}\right)=\left|x_{i}\right|^{-\beta_{i}}$ or $\gamma_{i} \in L^{\ell_{i}}(\mathbb{R})$

for some $0<\beta_{i}<1<\ell_{i}<+\infty, i=1,2$. 
Remark 1.2 (i) Note that condition (a) for $d=2$ is slightly stronger than Dalang's condition (1.2). In fact, when $d=2$, the paper [18] pointed out that Dalang's condition (1.2) is equivalent to

$$
\int_{|x| \leq 1} \ln \left(|x|^{-1}\right) \gamma(x) d x<\infty
$$

let $\ell^{\star}=\frac{\ell}{\ell-1}$ and $0<\varepsilon<1 / \ell^{\star}$, then there is some $\delta \in(0,1)$ and a constant $C_{\varepsilon}$ such that $\ln \left(|x|^{-1}\right) \leq C_{\varepsilon}|x|^{-\varepsilon}$ for any $|x| \leq \delta$, from which we deduce that

$$
\begin{aligned}
\int_{|x| \leq 1} \ln \left(|x|^{-1}\right) \gamma(x) d x \leq & \ln \left(\delta^{-1}\right) \int_{\delta<|x| \leq 1} \gamma(x) d x+C_{\varepsilon} \int_{|x| \leq \delta}|x|^{-\varepsilon} \gamma(x) d x \\
\leq & \ln \left(\delta^{-1}\right) \int_{\delta<|x| \leq 1} \gamma(x) d x \\
& +C_{\varepsilon}\|\gamma\|_{L^{\ell}\left(\mathbb{R}^{2}\right)}\left(\int_{|x| \leq \delta}|x|^{-\varepsilon \ell^{\star}} d x\right)^{1 / \ell^{\star}}<\infty .
\end{aligned}
$$

(ii) The case (c) in Hypothesis (H1) is a mixture of cases (a) and (b). Accordingly, more examples of the noise $\dot{W}$ arise. In the space variables, $W$ can behave like a fractional Brownian sheet with Hurst indices greater than $1 / 2$ in both directions, i.e. $\gamma\left(x_{1}, x_{2}\right)=\left|x_{1}\right|^{2 H_{1}-2}\left|x_{2}\right|^{2 H_{2}-2}$ for some $H_{1}, H_{2} \in(1 / 2,1)$.

(iii) For $d=1$ we just assume that $\gamma$ is a non-negative and non-negative definite measure on $\mathbb{R}$. In this case (see, for instance, Remark 10 of [11]) Dalang's condition is always satisfied.

Under Hypothesis (H1), we will state our first main result - the $L^{p}(\Omega)$ estimates of the Malliavin derivatives of $u(t, x)$. The first Malliavin derivative $D u(t, x)$ is a random element in the Hilbert space $\mathcal{H}$, the completion of $C_{c}^{\infty}\left(\mathbb{R}_{+} \times \mathbb{R}^{d}\right)$ under the inner product (1.3); as the space $\mathcal{H}$ contains generalized functions, it is not clear at first sight whether $(s, y) \longmapsto D_{s, y} u(t, x)$ is a (random) function. The higher-order Malliavin derivative $D^{m} u(t, x)$ is a random element in $\mathcal{H}^{\otimes m}$ for $m \geq 1$, see Sect. 2 for more details.

Let us first fix some notation.

Notation A (1) We write $a \lesssim b$ to mean $a \leq K b$ for some immaterial constant $K>0$. (2) We write $\|X\|_{p}=\left(\mathbb{E}\left[|X|^{p}\right]\right)^{1 / p}$ to denote the $L^{p}(\Omega)$-norm of $X$ for $p \in[1, \infty)$.

(3) When $p$ is a positive integer, we often write $z_{p}=\left(z_{1}, \ldots, z_{p}\right)$ for points in $\mathbb{R}_{+}^{p}$ or $\mathbb{R}^{d p}$, and $d z_{\boldsymbol{p}}=d z_{1} \cdots d z_{p}, \mu\left(d z_{\boldsymbol{p}}\right)=\mu\left(d z_{1}\right) \cdots \mu\left(d z_{p}\right)$. For a function $h:\left(\mathbb{R}_{+} \times \mathbb{R}^{d}\right)^{p} \rightarrow \mathbb{R}$ with $p \geq 2$, we often write

$$
h\left(\boldsymbol{s}_{\boldsymbol{p}}, \boldsymbol{y}_{\boldsymbol{p}}\right)=h\left(s_{1}, \ldots, s_{p}, y_{1}, \ldots, y_{p}\right)=h\left(s_{1}, y_{1}, \ldots, s_{p}, y_{p}\right)
$$

which shall not cause any confusion. For $m \in\{1, \ldots, p-1\}$ and $\left(\boldsymbol{s}_{\boldsymbol{m}}, \boldsymbol{y}_{\boldsymbol{m}}\right) \in \mathbb{R}_{+}^{m} \times \mathbb{R}^{d m}$, the expression $h\left(\boldsymbol{s}_{\boldsymbol{m}}, \boldsymbol{y}_{\boldsymbol{m}} ; \bullet\right)$ stands for the function 


$$
\begin{aligned}
& \left(t_{1}, x_{1}, \ldots, t_{p-m}, x_{p-m}\right) \mapsto h\left(s_{1}, y_{1}, \ldots, s_{m}, y_{m}, t_{1}, x_{1}, \ldots, t_{p-m}, x_{p-m}\right) \\
& \quad=h\left(\boldsymbol{s}_{\boldsymbol{m}}, \boldsymbol{y}_{\boldsymbol{m}} ; \boldsymbol{t}_{\boldsymbol{p}-\boldsymbol{m}}, \boldsymbol{x}_{\boldsymbol{p}-\boldsymbol{m}}\right) .
\end{aligned}
$$

Now, with the above notation in mind, we are in the position to state the first main result. $^{2}$

Theorem 1.3 Let $d \in\{1,2\}$ and suppose that Hypothesis $(\mathbf{H 1})$ holds if $d=2$. Then, for any $(t, x) \in \mathbb{R}_{+} \times \mathbb{R}^{d}$, the random variable $u(t, x)$ belongs to $\mathbb{D}^{\infty}$ (see Sect. 2.1 ). Moreover, for any integer $m \geq 1$, the $m$ th Malliavin derivative $D^{m} u(t, x)$ is a random symmetric function denoted by

$\left(\boldsymbol{s}_{\boldsymbol{m}}, \boldsymbol{y}_{\boldsymbol{m}}\right)=\left(s_{1}, y_{1}, \ldots, s_{m}, y_{m}\right) \longmapsto D_{s_{1}, y_{1}} D_{s_{2}, y_{2}} \ldots D_{s_{m}, y_{m}} u(t, x)=D_{\boldsymbol{s}_{\boldsymbol{m}}, y_{\boldsymbol{m}}}^{m} u(t, x)$,

and for any $p \in[2, \infty)$, we have, for almost all $\left(\boldsymbol{s}_{\boldsymbol{m}}, \boldsymbol{y}_{\boldsymbol{m}}\right) \in[0, t]^{m} \times \mathbb{R}^{m d}$,

$$
m ! \tilde{f}_{t, x, m}\left(\boldsymbol{s}_{\boldsymbol{m}}, \boldsymbol{y}_{\boldsymbol{m}}\right) \leq\left\|D_{\boldsymbol{s}_{\boldsymbol{m}}, \boldsymbol{y}_{\boldsymbol{m}}}^{m} u(t, x)\right\|_{p} \lesssim \widetilde{f}_{t, x, m}\left(\boldsymbol{s}_{\boldsymbol{m}}, \boldsymbol{y}_{\boldsymbol{m}}\right)
$$

where the constant in the upper bound only depends on $\left(p, t, \gamma_{0}, \gamma, m\right)$ and is increasing in $t$. Moreover, $D^{m} u(t, x)$ has a measurable modification.

Throughout this paper, we will work with the measurable modifications of $D u(t, x)$ and $D^{2} u(t, x)$ given by Theorem 1.3 , which are still denoted by $D u(t, x), D^{2} u(t, x)$ respectively.

In this paper, we will present two applications of Theorem 1.3. Our first application are quantitative central limit theorems (CLTs) for the spatial averages of the solution to (1.1), which have been elusive so far due to the temporal correlation of the noise preventing the use of Itô calculus approach. A novel ingredient to overcome this difficulty is the so-called second-order Gaussian Poincaré inequality in an improved form. We will address these CLT results in Sect. 1.1. While in Sect. 1.2, as the second application, we establish the absolute continuity of the law of the solution to equation (1.1) using the $L^{p}$-estimates of Malliavin derivatives that are crucial to establish a local version of Bouleau-Hirsch criterion [5].

\subsection{Gaussian fluctuation of spatial averages}

Spatial averages of SPDEs have recently attracted considerable interest. It was Huang, Nualart and Viitasaari who first studied the fluctuation of spatial statistics and established a central limit theorem for a nonlinear SPDE in [15]. More precisely, they considered the following one-dimensional stochastic heat equation

\footnotetext{
${ }^{2}$ In higher dimension $(d \geq 3)$, the fundamental wave solution is a uniform measure supported on certain surfaces, then the Malliavin derivative $D u(t, x)$ is expected to be merely a random measure instead of being a random function. In this case, the expression $D_{s, y} u(t, x)$ does not make sense; see also the recent article [34] for related discussions.
} 


$$
\frac{\partial u}{\partial t}=\frac{1}{2} \Delta u+\sigma(u) \dot{W}
$$

on $\mathbb{R}_{+} \times \mathbb{R}$, where $\dot{W}$ is a space-time Gaussian white noise, with constant initial condition $u(0, \bullet)=1$ and the nonlinearity $\sigma: \mathbb{R} \rightarrow \mathbb{R}$ is a Lipschitz function. In view of the localization property of its mild formulation (in the Walsh sense [43]),

$$
u(t, x)=1+\int_{0}^{t} \int_{\mathbb{R}} p_{t-s}(x-y) \sigma(u(s, y)) W(d s, d y),
$$

with $p_{t}$ denoting the heat kernel, ${ }^{3}$ one can regard $u(t, x)$ and $u(t, y)$ as weakly dependent random variables for $x, y$ far apart so that the integral

$$
\int_{-R}^{R}[u(t, x)-1] d x
$$

can be roughly understood as a sum of weakly dependent random variables. Therefore, it is very natural to expect Gaussian fluctuations when $R$ tends to infinity.

Let us stop now to briefly fix some notation to facilitate our discussion.

Notation B. (1) For $t>0$, we define, with $B_{R}:=\left\{x \in \mathbb{R}^{d}:|x| \leq R\right\}$,

$$
F_{R}(t):=\int_{B_{R}}[u(t, x)-1] d x \text { and } \sigma_{R}(t)=\sqrt{\operatorname{Var}\left(F_{R}(t)\right)} .
$$

(2) We write $f(R) \sim g(R)$ to mean that $f(R) / g(R)$ converges to some positive constant as $R \rightarrow \infty$.

(3) For two real random variables $X, Y$ with distribution measures $\mu, v$ respectively, the total variation distance between $X, Y$ (or $\mu, v)$ is defined to be

$$
d_{\mathrm{TV}}(X, Y)=\sup _{B}|\mu(B)-v(B)|,
$$

where the supremum runs over all Borel set $B \subset \mathbb{R}$. The total variation distance is well known to induce a stronger topology than that of convergence in distribution, see [25, Appendix C].

(4) We define the following quantities for future reference:

$\omega_{1}=2, \quad \omega_{2}=\pi, \quad$ and $\quad \kappa_{\beta, d}:=\int_{\mathbb{R}^{2 d}} d x d y|x-y|^{-\beta} \mathbf{1}_{B_{1}}(x) \mathbf{1}_{B_{1}}(y)$ for $\beta \in(0, d)$.

(5) For an integer $m \geq 1$ and $p \in[1, \infty)$, we say $F \in \mathbb{D}^{m, p}$ if $F$ is $m$-times Malliavin differentiable random variable in $L^{p}(\Omega)$ and $\mathbb{E}\left[\left\|D^{j} F\right\|_{\mathcal{H}^{\otimes j}}^{p}\right]<\infty$ for every $j=1, \ldots, m$; see Sect. 2.1 for more details.

Now let us illustrate the strategy in [15]: (For this reference, $d=1$ )

$\overline{{ }^{3} p_{t}(x)=(2 \pi t)^{-d / 2} e^{-|x|^{2} /(2 t)}}$ for $t>0$ and $x \in \mathbb{R}^{d}$; in (1.13), $d=1$. 
- The authors first rewrite $F_{R}(t)=\delta\left(V_{t, R}\right)$ with the random kernel

$$
V_{t, R}(s, y)=\sigma(u(s, y)) \int_{B_{R}} p_{t-s}(x-y) d x,
$$

where $\delta$ denotes the Skorokhod integral, the adjoint of the Malliavin derivative $D$.

- By standard computations, they obtained $\sigma_{R}^{2}(t) \sim R$.

- If $F=\delta(v) \in \mathbb{D}^{1,2}$ is a centered random variable with variance one, for some $v$ in the domain of $\delta$, the (univariate) Malliavin-Stein bound (see [15, Proposition 2.2]) ensures that $d_{\mathrm{TV}}(F, Z) \leq 2 \sqrt{\operatorname{Var}\left(\langle D F, v\rangle_{\mathcal{H}}\right)}$ for $Z \sim N(0,1)$.

- Combining the above points, one can see that the obtention of a quantitative CLT is reduced to the computation of $\operatorname{Var}\left(\left\langle D F_{R}(t), V_{t, R}\right\rangle_{\mathcal{H}}\right)$.

Because the driving noise is white in time as considered in [15], tools from Itô calculus (Clark-Ocone formula, Burkholder's inequality, etc.) are used to estimate the above variance term. It is proved in [15] that $d_{\mathrm{TV}}\left(F_{R}(t) / \sigma_{R}(t), Z\right) \lesssim R^{-1 / 2}$. Meanwhile, a multivariate Malliavin-Stein bound and similar computations lead to the convergence of the finite-dimensional distributions, which coupled with the tightness property gives a functional CLT for $\left\{R^{-1 / 2} F_{R}(t): t \in \mathbb{R}_{+}\right\}$.

The above general strategy has been adapted to various settings, see $[9,10,16,19$, $20,38]$ for the study of stochastic heat equations and see $[4,12,35]$ for the study of stochastic wave equations. All these references consider a Gaussian noise that is white in time. Nevertheless, when the Gaussian noise is colored in time, the mild formulation (1.13) cannot be interpreted in the Walsh-Itô sense. In this situation, only in the case $\sigma(u)=u$ the stochastic heat equation (1.12) (also known as the parabolic Anderson model) can be properly solved using Wiener chaos expansions, so that $F_{R}(t)$, defined in (1.14), can be expressed as an infinite sum of multiple Wiener integrals. With this well-known fact in mind, Nualart and Zheng [33] considered the parabolic Anderson model (i.e. (1.12) with $\sigma(u)=u$ ) on $\mathbb{R}_{+} \times \mathbb{R}^{d}$ such that $d \geq 1$, the initial condition is constant and the assumptions (i)-(ii) hold (see page 2). The main result of [33] is the chaotic CLT that is based on the fourth moment theorems [30,37]. When, additionally, $\gamma$ is a finite measure, the authors of [33] established $\sigma_{R}(t) \sim R^{d / 2}$ and a functional CLT for the process $R^{-d / 2} F_{R}$; they also considered the case where $\gamma(x)=|x|^{-\beta}$, for some $\beta \in(0,2 \wedge d)$, is the Riesz kernel, and obtain the corresponding CLT results. As pointed out in the paper [33], due to the homogeneity of the underlying Gaussian noise, the solution $u$ to (1.12) can be regarded as the functional of a stationary Gaussian random field so that, with the Breuer-Major theorem [6] in mind, it is natural to study Gaussian fluctuations for the problems (1.12) and (1.1). Note that the constant initial condition makes the solution stationary in space and, in fact it is spatially ergodic (see $[10,36])$. At last, let us mention the paper [32] in which chaotic CLT was used to study the parabolic Anderson model driven by a colored Gaussian noise that is rough in space. However, let us point out that the aforementioned methods fail to provide the rate of convergence when the noise is colored in time. 
In this paper, we bring in a novel ingredient—-the second-order Gaussian Poincaré inequality ${ }^{4}$ - to reach quantitative CLT results for the hyperbolic Anderson model (1.1). Let us first state our main result.

Theorem 1.4 Let $u$ denote the solution to the hyperbolic Anderson model (1.1) and recall the definition of $F_{R}(t)$ and $\sigma_{R}(t)$ from (1.14). Let $Z \sim N(0,1)$ be the standard normal random variable. We assume that $\gamma_{0}$ is not identically zero meaning

$$
\left\|\gamma_{0}\right\|_{L^{1}([0, \varepsilon])}>0 \text { for any } \varepsilon \in(0,1) \text {. }
$$

Then the following statements hold true:

(1) Suppose that $0<\gamma\left(\mathbb{R}^{d}\right)<\infty$ if $d=1$ and $\gamma \in L^{1}\left(\mathbb{R}^{d}\right) \cap L^{\ell}\left(\mathbb{R}^{d}\right)$ for some $\ell>1$ if $d=2$. Then,

$$
\sigma_{R}(t) \sim R^{d / 2} \text { and } d_{\mathrm{TV}}\left(F_{R}(t) / \sigma_{R}(t), Z\right) \lesssim R^{-d / 2}
$$

Moreover, as $R \rightarrow \infty$, the process $\left\{R^{-d / 2} F_{R}(t): t \in \mathbb{R}_{+}\right\}$converges weakly in the space of continuous functions $C\left(\mathbb{R}_{+}\right)$to a centered Gaussian process $\mathcal{G}$ with covariance structure

$$
\mathbb{E}[\mathcal{G}(t) \mathcal{G}(s)]=\omega_{d} \sum_{p \geq 1} p ! \int_{\mathbb{R}^{d}}\left\langle\widetilde{f}_{t, x, p}, \widetilde{f}_{s, 0, p}\right\rangle_{\mathcal{H}^{\otimes p}} d x
$$

fort,$s \in \mathbb{R}_{+}$. Here $\omega_{1}=2, \omega_{2}=\pi$ and $\widetilde{f}_{t, x, p}$ are introduced in (1.16) and (1.9), respectively. The convergence of the series in (1.18) is part of the conclusion.

(2) Suppose $d \in\{1,2\}$ and $\gamma(x)=|x|^{-\beta}$ for some $\beta \in(0,2 \wedge d)$. Then,

$$
\sigma_{R}(t) \sim R^{d-\frac{\beta}{2}} \text { and } d_{\mathrm{TV}}\left(F_{R}(t) / \sigma_{R}(t), Z\right) \lesssim R^{-\beta / 2}
$$

Moreover, as $R \rightarrow \infty$, the process $\left\{R^{-d+\frac{\beta}{2}} F_{R}(t): t \in \mathbb{R}_{+}\right\}$converges weakly in the space $C\left(\mathbb{R}_{+}\right)$to a centered Gaussian process $\mathcal{G}_{\beta}$ with the covariance structure

$$
\mathbb{E}\left[\mathcal{G}_{\beta}(t) \mathcal{G}_{\beta}(s)\right]=\kappa_{\beta, d} \int_{0}^{t} d r \int_{0}^{s} d r^{\prime} \gamma_{0}\left(r-r^{\prime}\right)(t-r)\left(s-r^{\prime}\right),
$$

for $t, s \in \mathbb{R}_{+}$. Here the quantity $\kappa_{\beta, d}$ is introduced in (1.16).

\footnotetext{
4 The use of second-order Gaussian Poincaré inequality for obtaining CLT on a Gaussian space is one of the central techniques in the Malliavin-Stein approach; for example, in the recent paper [13], Dunlap et al. have used this Poincaré inequality to investigate the Gaussian fluctuation of the KPZ in dimension three and higher. We remark here that we can not directly apply this inequality because of the complicated correlation structure of the underlying Gaussian homogeneous noise, while the underlying Gaussian noise in [13] is white in time and smooth in space so that they can directly apply the version from [26]. In this article, we have established a quite involved variant of second-order Poincaré inequality, which is tailor-made for our applications.
} 
(3) Suppose $d=2$ and $\gamma\left(x_{1}, x_{2}\right)=\gamma_{1}\left(x_{1}\right) \gamma_{2}\left(x_{2}\right)$ such that one of the following two conditions holds:

$$
\left\{\begin{array}{l}
\left(\mathrm{a}^{\prime}\right) \gamma_{i}\left(x_{i}\right)=\left|x_{i}\right|^{-\beta_{i}} \text { for some } \beta_{i} \in(0,1), i=1,2 ; \\
\left(\mathrm{b}^{\prime}\right) \gamma_{1} \in L^{\ell}(\mathbb{R}) \cap L^{1}(\mathbb{R}) \text { and } \gamma_{2}\left(x_{2}\right)=\left|x_{2}\right|^{-\beta} \text { for some } 0<\beta<1<\ell<\infty .
\end{array}\right.
$$

Then,

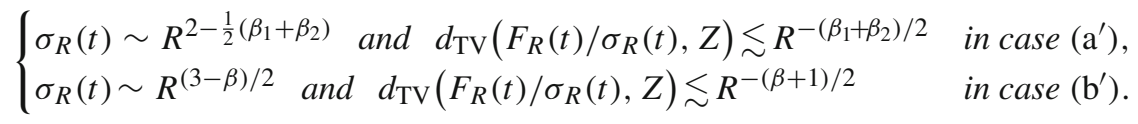

Moreover, as $R \rightarrow \infty$, in case $\left(a^{\prime}\right)$, the process $\left\{R^{-2+\frac{\beta_{1}+\beta_{2}}{2}} F_{R}(t): t \in \mathbb{R}_{+}\right\}$ converges weakly in the space $C\left(\mathbb{R}_{+}\right)$to a centered Gaussian process $\mathcal{G}_{\beta_{1}, \beta_{2}}$ with the covariance structure

$\mathbb{E}\left[\mathcal{G}_{\beta_{1}, \beta_{2}}(t) \mathcal{G}_{\beta_{1}, \beta_{2}}(s)\right]=K_{\beta_{1}, \beta_{2}} \int_{0}^{t} d r \int_{0}^{s} d r^{\prime} \gamma_{0}\left(r-r^{\prime}\right)(t-r)\left(s-r^{\prime}\right)$,

for $t, s \in \mathbb{R}_{+}$, where

$K_{\beta_{1}, \beta_{2}}:=\int_{\mathbb{R}^{4}} \mathbf{1}_{\left\{x_{1}^{2}+x_{2}^{2} \leq 1\right\}} \mathbf{1}_{\left\{y_{1}^{2}+y_{2}^{2} \leq 1\right\}}\left|x_{1}-y_{1}\right|^{-\beta_{1}}\left|x_{2}-y_{2}\right|^{-\beta_{2}} d x_{1} d x_{2} d y_{1} d y_{2} ;$

and in case $\left(b^{\prime}\right)$, the process $\left\{R^{\frac{\beta-3}{2}} F_{R}(t): t \in \mathbb{R}_{+}\right\}$converges weakly in the space $C\left(\mathbb{R}_{+}\right)$to a centered Gaussian process $\widehat{\mathcal{G}}_{\beta}$ with the covariance structure

$$
\mathbb{E}\left[\widehat{\mathcal{G}}_{\beta}(t) \widehat{\mathcal{G}}_{\beta}(s)\right]=\gamma_{1}(\mathbb{R}) \mathcal{L}_{\beta} \int_{0}^{t} d r \int_{0}^{s} d r^{\prime} \gamma_{0}\left(r-r^{\prime}\right)(t-r)\left(s-r^{\prime}\right)
$$

for $t, s \in \mathbb{R}_{+}$, where

$$
\mathcal{L}_{\beta}:=\int_{\mathbb{R}^{3}} d x_{1} d x_{2} d x_{3} \mathbf{1}_{\left\{x_{1}^{2}+x_{2}^{2} \leq 1\right\}} \mathbf{1}_{\left\{x_{1}^{2}+x_{3}^{2} \leq 1\right\}}\left|x_{2}-x_{3}\right|^{-\beta}
$$

For the above functional convergences, we specify that the space $C\left(\mathbb{R}_{+}\right)$is equipped with the topology of uniform convergence on compact sets.

Remark 1.5 (1) Note that the case when $\gamma(x)=\gamma_{1}\left(x_{1}\right) \gamma_{2}\left(x_{2}\right)$ with $\gamma_{i} \in L^{\ell_{i}}(\mathbb{R}) \cap$ $L^{1}(\mathbb{R})$ for some $\ell_{i}>1, i=1,2$, is covered in part (1). Indeed, suppose that $\ell_{1} \geq$ $\ell_{2}$, then by Hölder's inequality, $\gamma_{1} \in L^{\ell_{1}}(\mathbb{R}) \cap L^{1}(\mathbb{R})$ implies $\gamma_{1} \in L^{\ell_{2}}(\mathbb{R}) \cap L^{1}(\mathbb{R})$ and hence $\gamma \in L^{\ell_{2}}\left(\mathbb{R}^{2}\right) \cap L^{1}\left(\mathbb{R}^{2}\right)$.

(ii) The rate of convergence can also be described using other common distances such as the Wasserstein distance and the Kolmogorov distance; see [25, Appendix C]. 
(iii) The variance orders and the rates in parts (1) and (2) of Theorem 1.4 are consistent with previous work on stochastic wave equations, see $[4,12,35]$. The setting in part (3) is new. As we will see shortly, our strategy is quite different from that in these papers.

Now, let us briefly explain our strategy and begin with the Gaussian Poincaré inequality. For $F \in \mathbb{D}^{1,2}$, the Gaussian Poincaré inequality (see e.g. [14] or (2.12)) ensures that

$$
\operatorname{Var}(F) \leq \mathbb{E}\left[\|D F\|_{\mathcal{H}}^{2}\right] \text { with equality if and only if } F \text { is Gaussian, }
$$

that is, if $D F$ is small, then the random variable $F$ has necessarily small fluctuations. In the paper [8], Chatterjee pointed out that for $F=f\left(X_{1}, \ldots, X_{d}\right)$ with $X_{1}, \ldots, X_{d}$ i.i.d. $N(0,1)$ and $f$ twice differentiable, $F$ is close in total variation distance to a normal distribution with matched mean and variance if the Hessian matrix Hess $f\left(X_{1}, \ldots, X_{d}\right)$ is negligible, roughly speaking. This is known as the secondorder Gaussian Poincaré inequality. In what follows, we state the infinite-dimensional version of this inequality due to Nourdin, Peccati and Reinert; see the paper [26] as well as the book [25]. ${ }^{5}$

Proposition 1.6 Let $F$ be a centered element of $\mathbb{D}^{2,4}$ such that $\mathbb{E}\left[F^{2}\right]=\sigma^{2}>0$ and let $Z \sim N\left(0, \sigma^{2}\right)$. Then,

$$
d_{\mathrm{TV}}(F, Z) \leq \frac{3}{\sigma^{2}}\left(\mathbb{E}\left[\left\|D^{2} F \otimes_{1} D^{2} F\right\|_{\mathcal{H}^{\otimes 2}}^{2}\right]\right)^{1 / 4}\left(\mathbb{E}\left[\|D F\|_{\mathcal{H}}^{4}\right]\right)^{1 / 4}
$$

where $D^{2} F \otimes_{1} D^{2} F$ denotes the 1-contraction between $D^{2} F$ and itself (see 2.10).

It has been known that this inequality usually gives sub-optimal rate. In the recent work [42] by Vidotto, she provided an improved version of the above inequality, where she considered an $L^{2}$-based Hilbert space $\mathcal{H}=L^{2}(A, v)$ with $v$ a diffusive measure (nonnegative, $\sigma$-finite and non-atomic) on some measurable space $A$. Let us state this result for the convenience of readers.

Theorem 1.7 (Theorem 2.1 in [42]) Let $F \in \mathbb{D}^{2,4}$ with mean zero and variance $\sigma^{2}>0$ and let $Z \sim N\left(0, \sigma^{2}\right)$. Suppose $\mathcal{H}=L^{2}(A, v)$ with $v$ a diffusive measure on some measurable space A. Then,

$$
\begin{aligned}
& d_{\mathrm{TV}}(F, Z) \\
& \leq \frac{4}{\sigma^{2}}\left[\int_{A \times A} \sqrt{\mathbb{E}\left[\left(D^{2} F \otimes_{1} D^{2} F\right)^{2}(x, y)\right] \times \mathbb{E}\left[(D F)^{2}(x)(D F)^{2}(y)\right]} v(d x) v(d y)\right]^{\frac{1}{2}} .
\end{aligned}
$$

The proof of the above inequality follows from the general Malliavin-Stein bound

$$
d_{\mathrm{TV}}(F, Z) \leq \frac{2}{\sigma^{2}} \mathbb{E}\left(\left|\sigma^{2}-\left\langle D F,-D L^{-1} F\right\rangle_{\mathcal{H}}\right|\right)
$$

\footnotetext{
5 Note that there is a typo in equation (5.3.2) of [25]: We have $E\left[\|D F\|_{\mathcal{H}}^{4}\right]^{1 / 4}$ instead of $E\left[\left\|D^{2} F\right\|_{\mathcal{H}}^{4}\right]^{1 / 4}$.
} 
(see [25, equation (5.1.4)] $)^{6}$ and Vidotto's new bound of

$$
\mathbb{E}\left[\left(\operatorname{Cov}(F, G)-\left\langle D F,-D L^{-1} G\right\rangle_{\mathcal{H}}\right)^{2}\right] \text { for centered } F, G \in \mathbb{D}^{2,4}
$$

(see [42, Proposition 3.2]), where $L^{-1}$ is the pseudo-inverse of the Ornstein-Uhlenbeck operator $L$; see Sect. 2.1 for the definitions.

Recall that our Hilbert space $\mathcal{H}$ is the completion of $C_{c}^{\infty}\left(\mathbb{R}_{+} \times \mathbb{R}^{d}\right)$ under the inner product (1.3). The Hilbert space $\mathcal{H}$ contains generalized functions, but fortunately the objects $D^{2} u(t, x), D u(t, x)$ are random functions in view of Theorem 1.3. By adapting Vidotto's proof to our setting, we have the following version of second-order Gaussian Poincaré inequality. Note we write $f \in\left|\mathcal{H}^{\otimes p}\right|$ to mean $f$ is a real valued function and $\bullet \mapsto|f(\bullet)|$ belongs to $\mathcal{H}^{\otimes p}$.

Proposition 1.8 If $F \in \mathbb{D}^{2,4}$ has mean zero and variance $\sigma^{2} \in(0, \infty)$ such that with probability $1, D F \in|\mathcal{H}|$ and $D^{2} F \in\left|\mathcal{H}^{\otimes 2}\right|$, then

$$
d_{\mathrm{TV}}(F, Z) \leq \frac{4}{\sigma^{2}} \sqrt{\mathcal{A}},
$$

where $Z \sim N\left(0, \sigma^{2}\right)$ and

$$
\begin{aligned}
\mathcal{A}:= & \int_{\mathbb{R}_{+}^{6} \times \mathbb{R}^{6 d}} d r d r^{\prime} d s d s^{\prime} d \theta d \theta^{\prime} d z d z^{\prime} d y d y^{\prime} d w d w^{\prime} \gamma_{0}\left(\theta-\theta^{\prime}\right) \gamma_{0}\left(s-s^{\prime}\right) \gamma_{0}\left(r-r^{\prime}\right) \\
& \times \gamma\left(z-z^{\prime}\right) \gamma\left(w-w^{\prime}\right) \gamma\left(y-y^{\prime}\right)\left\|D_{r, z} D_{\theta, w} F\right\|_{4}\left\|D_{s, y} D_{\theta^{\prime}, w^{\prime}} F\right\|_{4} \| \\
& \times D_{r^{\prime}, z^{\prime}} F\left\|_{4}\right\| D_{s^{\prime}, y^{\prime}} F \|_{4} .
\end{aligned}
$$

As mentioned before, Proposition 1.8 will follow from the Malliavin-Stein bound (1.26) and Cauchy-Schwarz inequality, taking into account that, by the duality relation (2.5), we have that $\mathbb{E}\left(\left\langle D F,-D L^{-1} F\right\rangle_{\mathcal{H}}\right)=\mathbb{E}\left[F^{2}\right]=\sigma^{2}$. Indeed, we can write

$$
\begin{aligned}
d_{\mathrm{TV}}(F, Z) & \leq \frac{2}{\sigma^{2}} \mathbb{E}\left(\left|\sigma^{2}-\left\langle D F,-D L^{-1} F\right\rangle_{\mathcal{H}}\right|\right) \leq \frac{2}{\sigma^{2}} \sqrt{\operatorname{Var}\left(\left\langle D F,-D L^{-1} F\right\rangle_{\mathcal{H}}\right)} \\
& \leq \frac{4}{\sigma^{2}} \sqrt{\mathcal{A}} \text { by Proposition } 1.9 \text { below. }
\end{aligned}
$$

Proposition 1.9 If $F, G \in \mathbb{D}^{2,4}$ have mean zero such that with probability one, $D F, D G \in|\mathcal{H}|$ and $D^{2} F, D^{2} G \in\left|\mathcal{H}^{\otimes 2}\right|$, then

$$
\operatorname{Var}\left(\left\langle D F,-D L^{-1} G\right\rangle_{\mathcal{H}}\right)=\mathbb{E}\left[\left(\operatorname{Cov}(F, G)-\left\langle D F,-D L^{-1} G\right\rangle_{\mathcal{H}}\right)^{2}\right] \leq 2 A_{1}+2 A_{2}
$$

\footnotetext{
${ }^{6}$ Unlike in [25], we do not assume $F$ to have a density; in fact, it suffices to use [44, Proposition 2.1.1] and [25, (5.1.1)] to establish [25, Eq. (5.1.4)]. 
where

$$
\begin{aligned}
A_{1}:= & \int_{\mathbb{R}_{+}^{6} \times \mathbb{R}^{6 d}} d r d r^{\prime} d s d s^{\prime} d \theta d \theta^{\prime} d z d z^{\prime} d y d y^{\prime} d w d w^{\prime} \\
& \times \gamma_{0}\left(\theta-\theta^{\prime}\right) \gamma_{0}\left(s-s^{\prime}\right) \gamma_{0}\left(r-r^{\prime}\right) \gamma\left(z-z^{\prime}\right) \gamma\left(w-w^{\prime}\right) \gamma\left(y-y^{\prime}\right) \\
& \times\left\|D_{r, z} D_{\theta, w} F\right\|_{4}\left\|D_{s, y} D_{\theta^{\prime}, w^{\prime}} F\right\|_{4}\left\|D_{r^{\prime}, z^{\prime}} G\right\|_{4}\left\|D_{s^{\prime}, y^{\prime}} G\right\|_{4}
\end{aligned}
$$

and $A_{2}$ is defined by switching the positions of $F, G$ in the definition of $A_{1}$.

For the sake of completeness, we sketch the proof of Proposition 1.9 in Appendix A.2. Once we have the information on the growth order of $\sigma_{R}(t)$, we can apply Theorem 1.3 and Proposition 1.9 to obtain the error bounds in Theorem 1.4. The proof of Theorem 1.4 will be given in Sect. 4: In Sect. 4.1, we will establish the limiting covariance structure, which will be used to obtain the quantitative CLTs in Sect. 4.2; Proposition 1.9, combined with a multivariate Malliavin-Stein bound (see e.g. [25, Theorem 6.1.2]), also gives us easy access to the convergence of finite-dimensional distributions (f.d.d. convergence) for part (1), while in the other parts, the f.d.d. convergence follows easily from the dominance of the first chaotic component of $F_{R}(t)$; finally in Sect. 4.3, we establish the functional CLT by showing the required tightness, which will follow by verifying the well-known criterion of Kolmogorov-Chentsov (see e.g. [17, Corollary 16.9]).

\subsection{Absolute continuity of the law of the solution to Eq. (1.1)}

In this part, we fix the following extra hypothesis on the correlation kernels $\gamma_{0}, \gamma$.

(H2) $\left\{\begin{array}{l}\gamma_{0}=\mathcal{F} \mu_{0} \text { and } \gamma=\mathcal{F} \mu \text {, where } \mu_{0}, \mu \text { are nonnegative tempered measures } \\ \text { and have strictly positive densities with respect to the Lebesgue measure. }\end{array}\right.$

The following is the main result of this section.

Theorem 1.10 Let $d \in\{1,2\}$ and assume that Hypothesis (H2) holds. In addition, assume that Hypothesis (H1) holds if $d=2$. Let $u$ be the solution to (1.1). For any $t>0$ and $x \in \mathbb{R}^{d}$, the law of $u(t, x)$ restricted to the set $\mathbb{R} \backslash\{0\}$ is absolutely continuous with respect to the Lebesgue measure on $\mathbb{R} \backslash\{0\}$.

Let us sketch the proof of Theorem 1.10. In view of the Bouleau-Hirsch criterion for absolute continuity (see [5]), it suffices to prove that for each $m \geq 1$,

$$
\|D u(t, x)\|_{\mathcal{H}}>0 \quad \text { a.s. on } \Omega_{m},
$$

where $\Omega_{m}=\{|u(t, x)| \geq 1 / m\}$. Notice that

$$
\|D u(t, x)\|_{\mathcal{H}}^{2}=\int_{0}^{t} \int_{0}^{t} \gamma_{0}(r-s)\left\langle D_{r, \bullet} u(t, x), D_{s, \bullet} u(t, x)\right\rangle_{0} d r d s,
$$


where $\mathcal{P}_{0}$ is the completion of $C_{c}^{\infty}\left(\mathbb{R}^{d}\right)$ with respect to the inner product $\langle\cdot, \cdot\rangle_{0}$ introduced in (2.1). The usual approach to show the positivity of this norm is to get a lower bound for this integral by integrating on a small interval $[t-\delta, t]^{2}$ and use that, for $r$ close to $t, D_{r, y} u(t, x)$ behaves as $G_{t-r}(x-y) u(s, y)$ (see, e.g., [31]). However, for $r \neq s$, the inner product $\left\langle D_{r, \bullet} u(t, x), D_{s, \bullet} u(t, x)\right\rangle_{0}$ is not necessarily non-negative. Our strategy to overcome this difficulty consists in making use of Hypothesis (H2) in order to show that

$$
\int_{0}^{t}\left\|D_{r, \bullet} u(t, x)\right\|_{0}^{2} d r>0 \text { implies }\|D u(t, x)\|_{\mathcal{H}}>0 \text { (see Lemma A.1). }
$$

This allows us to reduce the problem to the non-degeneracy of $\int_{t-\delta}^{t}\left\|D_{r, \bullet} u(t, x)\right\|_{0}^{2} d r$ for $\delta$ small enough, which can be handled by the usual arguments. At this point, we will make use of the estimates provided in Theorem 1.3.

For $d=1$, Theorem 1.10 was proved in [2] under stronger assumptions on the covariance structure. The result in Theorem 1.10 for $d=2$ is new. Indeed, the study of the existence (and smoothness) of the density for the stochastic wave equation has been extensively revisited over the last three decades. We refer the readers to [7,22,23,31,39-41]. In all these articles, the authors considered a stochastic wave equation of the form

$$
\frac{\partial^{2} u}{\partial t^{2}}(t, x)=\Delta u(t, x)+b(u(t, x))+\sigma(u(t, x)) \dot{\mathfrak{X}}(t, x),
$$

on $\mathbb{R}_{+} \times \mathbb{R}^{d}$, with $d \geq 1$. Here, $\dot{\mathfrak{X}}$ denotes a space-time white noise in the case $d=1$, or a Gaussian noise that is white in time and has a spatially homogeneous correlation (slightly more general than that of $W$ ) in the case $d \geq 2$. The functions $b, \sigma$ are usually assumed to be globally Lipschitz, and such that the following non-degeneracy condition is fulfilled: $|\sigma(z)| \geq C>0$, for all $z \in \mathbb{R}$. The temporal nature of the noise $\dot{\mathfrak{X}}$ made possible to interpret the solution in the classical Dalang-Walsh sense, making use of all needed martingale techniques. The first attempt to consider a Gaussian noise that is colored in time was in the paper [2], where the hyperbolic Anderson model with spatial dimension one was considered. As mentioned above, in that paper the existence of density was proved under a slightly stronger assumption than Hypothesis (H2).

The rest of this paper is organized as follows. Section 2 contains preliminary results and the proofs of our main results-Theorems 1.3, 1.4 and 1.10_-are given in Sects. 3, 4 and 5 , respectively.

\section{Preliminary results}

This section is devoted to presenting some basic elements of the Malliavin calculus and collecting some preliminary results that will be needed in the sequel. 


\subsection{Basic Malliavin calculus}

Recall that the Hilbert space $\mathcal{H}$ is the completion of $C_{c}^{\infty}\left(\mathbb{R}_{+} \times \mathbb{R}^{d}\right)$ under the inner product (1.3) that can be written as

$$
\langle\psi, \phi\rangle_{\mathcal{H}}=\int_{\mathbb{R}_{+}^{2}} d s d t \gamma_{0}(t-s)\langle\psi(t, \bullet), \phi(s, \bullet)\rangle_{0} \quad \text { for } \psi, \phi \in C_{c}^{\infty}\left(\mathbb{R}_{+} \times \mathbb{R}^{d}\right),
$$

where

$$
\langle h, g\rangle_{0}=\int_{\mathbb{R}^{2 d}} d z d z^{\prime} \gamma\left(z-z^{\prime}\right) h(z) g\left(z^{\prime}\right) .
$$

As defined in Sect. 1.2, we denote by $\mathcal{P}_{0}$ the completion of $C_{c}^{\infty}\left(\mathbb{R}^{d}\right)$ with respect to the inner product $\langle h, g\rangle_{0}$. Let $\left|\mathcal{P}_{0}\right|$ be the set of measurable functions $h: \mathbb{R}^{d} \rightarrow \mathbb{R}$ such that

$$
\int_{\mathbb{R}^{2 d}} d z d z^{\prime} \gamma\left(z-z^{\prime}\right)|h|(z)|h|\left(z^{\prime}\right)<\infty
$$

Then $\left|\mathcal{P}_{0}\right| \subset \mathcal{P}_{0}$ and for $h \in\left|\mathcal{P}_{0}\right|,\|h\|_{0}^{2}=\int_{\mathbb{R}^{2 d}} d z d z^{\prime} \gamma\left(z-z^{\prime}\right) h(z) h\left(z^{\prime}\right)$. We define the space $|\mathcal{H}|$ in a similar way. For $h, g \in C_{c}^{\infty}\left(\mathbb{R}^{d}\right)$ we can express (2.1) using the Fourier transform:

$$
\langle h, g\rangle_{0}=\int_{\mathbb{R}^{d}} \mu(d \xi) \mathcal{F} h(\xi) \overline{\mathcal{F} g(\xi)} .
$$

The Parseval-type relation (2.3) also holds for functions $h, g \in L^{1}\left(\mathbb{R}^{d}\right) \cap\left|\mathcal{P}_{0}\right|$.

For every integer $p \geq 1, \mathcal{H}^{\otimes p}$ and $\mathcal{H}^{\odot p}$ denote the $p$ th tensor product of $\mathcal{H}$ and its symmetric subspace, respectively. For example, $f_{t, x, n}$ in (1.8) belongs to $\mathcal{H}^{\otimes n}$ and $\widetilde{f}_{t, x, n} \in \mathcal{H}^{\odot n}$; we also have $f \otimes g \in \mathcal{H}^{\otimes(n+m)}$, provided $f \in \mathcal{H}^{\otimes m}$ and $g \in \mathcal{H}^{\otimes n}$; see [25, Appendix B] for more details.

Fix a probability space $(\Omega, \mathcal{B}, \mathbb{P})$, on which we can construct the isonormal Gaussian process associated to the Gaussian noise $\dot{W}$ in $(1.1)$ that we denote by $\{W(\phi): \phi \in \mathcal{H}\}$. That is, $\{W(\phi): \phi \in \mathcal{H}\}$ is a centered Gaussian family of realvalued random variables defined on $(\Omega, \mathcal{B}, \mathbb{P})$ such that $\mathbb{E}[W(\psi) W(\phi)]=\langle\psi, \phi\rangle_{\mathcal{H}}$ for any $\psi, \phi \in \mathcal{H}$. We will take $\mathcal{B}$ to be the $\sigma$-algebra $\sigma\{W\}$ generated by the family of random variables $\left\{W(h): h \in C_{c}^{\infty}\left(\mathbb{R}_{+} \times \mathbb{R}^{d}\right)\right\}$.

In the sequel, we recall some basics on Malliavin calculus from the books [25,27].

Let $C_{\text {poly }}^{\infty}\left(\mathbb{R}^{n}\right)$ denote the space of smooth functions with all their partial derivatives having at most polynomial growth at infinity and let $\mathcal{S}$ denote the set of simple smooth functionals of the form

$$
F=f\left(W\left(h_{1}\right), \ldots, W\left(h_{n}\right)\right) \text { for } f \in C_{\text {poly }}^{\infty}\left(\mathbb{R}^{n}\right) \text { and } h_{i} \in \mathcal{H}, 1 \leq i \leq n
$$


For such a random variable $F$, its Malliavin derivative $D F$ is the $\mathcal{H}$-valued random variable given by

$$
D F=\sum_{i=1}^{n} \frac{\partial f}{\partial x_{i}}\left(W\left(h_{1}\right), \ldots, W\left(h_{n}\right)\right) h_{i}
$$

And similarly its $m$ th Malliavin derivative $D^{m} F$ is the $\mathcal{H}^{\otimes m}$-valued random variable given by

$$
D^{m} F=\sum_{i_{1}, \ldots, i_{m}=1}^{n} \frac{\partial^{m} f}{\partial x_{i_{1}} \cdots \partial x_{i_{m}}}\left(W\left(h_{1}\right), \ldots, W\left(h_{n}\right)\right) h_{i_{1}} \otimes \cdots \otimes h_{i_{m}}
$$

which is an element in $L^{p}\left(\Omega ; \mathcal{H}^{\odot m}\right)$ for any $p \in[1, \infty)$. It is known that the space $\mathcal{S}$ is dense in $L^{p}(\Omega, \sigma\{W\}, \mathbb{P})$ and

$$
D^{m}: \mathcal{S} \longrightarrow L^{p}\left(\Omega ; \mathcal{H}^{\odot m}\right)
$$

is closable for any $p \in[1, \infty)$; see e.g. Lemma 2.3.1 and Proposition 2.3.4 in [25]. Let $\mathbb{D}^{m, p}$ be the closure of $\mathcal{S}$ under the norm

$$
\begin{aligned}
\|F\|_{\mathbb{D}^{m, p}} & =\left(\mathbb{E}\left[|F|^{p}\right]+\mathbb{E}\left[\|D F\|_{\mathcal{H}}^{p}\right]+\cdots+\mathbb{E}\left[\left\|D^{m} F\right\|_{\mathcal{H}^{\otimes m}}^{p}\right]\right)^{1 / p} \text { and } \\
\text { let } \mathbb{D}^{\infty}: & =\bigcap_{m, p \geq 1} \mathbb{D}^{m, p} .
\end{aligned}
$$

Now, let us introduce the adjoint of the derivative operator $D^{m}$. Let $\operatorname{Dom}\left(\delta^{m}\right)$ be the set of random variables $v \in L^{2}\left(\Omega ; \mathcal{H}^{\otimes m}\right)$ such that there is a constant $C_{v}>0$ for which

$$
\left|\mathbb{E}\left[\left\langle D^{m} F, v\right\rangle_{\mathcal{H}^{\otimes m}}\right]\right| \leq C_{v}\|F\|_{2} \text { for all } F \in \mathcal{S}
$$

By Riesz representation theorem, there is a unique random variable, denoted by $\delta^{m}(v)$, such that the following duality relationship holds:

$$
\mathbb{E}\left[F \delta^{m}(v)\right]=\mathbb{E}\left[\left\langle D^{m} F, v\right\rangle_{\mathcal{H}^{\otimes m}}\right]
$$

Equality (2.5) holds for all $v \in \operatorname{Dom}\left(\delta^{m}\right)$ and all $F \in \mathbb{D}^{m, 2}$. In the simplest case when $F=f(W(h))$ with $h \in \mathcal{H}$ and $f \in C_{\text {poly }}^{1}(\mathbb{R})$, we have $\delta(h)=W(h) \sim N\left(0,\|h\|_{\mathcal{H}}^{2}\right)$ and equality (2.5) reduces to

$$
\mathbb{E}[f(W(h)) W(h)]=\mathbb{E}\left[f^{\prime}(W(h))\right]\|h\|_{\mathcal{H}}^{2},
$$


which is exactly part of the Stein's lemma recalled below: For $\sigma \in(0, \infty)$ and an integrable random variable $Z$, Stein's lemma (see e.g. [25, Lemma 3.1.2]) asserts that

$$
Z \sim N\left(0, \sigma^{2}\right) \text { if and only if } \mathbb{E}[Z f(Z)]=\sigma^{2} \mathbb{E}\left[f^{\prime}(Z)\right],
$$

for any differentiable function $f: \mathbb{R} \rightarrow \mathbb{R}$ such that the above expectations are finite. The operator $\delta$ is often called the Skorokhod integral since in the case of the Brownian motion, it coincides with an extension of the Itô integral introduced by Skorokhod, see e.g. [29]. Then we can say $\operatorname{Dom}\left(\delta^{m}\right)$ is the space of Skorokhod integrable random variables with values in $\mathcal{H}^{\otimes m}$.

The Wiener-Itô chaos decomposition theorem asserts that $L^{2}(\Omega, \sigma\{W\}, \mathbb{P})$ can be written as a direct sum of mutually orthogonal subspaces:

$$
L^{2}(\Omega, \sigma\{W\}, \mathbb{P})=\bigoplus_{n \geq 0} \mathbb{C}_{n}^{W}
$$

where $\mathbb{C}_{0}^{W}$, identified as $\mathbb{R}$, is the space of constant random variables and $\mathbb{C}_{n}^{W}=$ $\left\{\delta^{n}(h): h \in \mathcal{H}^{\otimes n}\right.$ is deterministic $\}$, for $n \geq 1$, is called the $n$th Wiener chaos associated to $W$. Note that the first Wiener chaos consists of centered Gaussian random variables. When $h \in \mathcal{H}^{\otimes n}$ is deterministic, we write $I_{n}(h)=\delta^{n}(h)$ and we call it the $n$th multiple integral of $h$ with respect to $W$. By the symmetry in (2.4) and the duality relation $(2.5), \delta^{n}(h)=\delta^{n}(\widetilde{h})$ with $\widetilde{h}$ the canonical symmetrization of $h$, so that we have $I_{n}(h)=I_{n}(\widetilde{h})$ for any $h \in \mathcal{H}^{\otimes n}$. The above decomposition can be rephrased as follows. For any $F \in L^{2}(\Omega, \sigma\{W\}, \mathbb{P})$,

$$
F=\mathbb{E}[F]+\sum_{n \geq 1} I_{n}\left(f_{n}\right),
$$

with $f_{n} \in \mathcal{H}^{\odot n}$ uniquely determined for each $n \geq 1$. Moreover, the (modified) isometry property holds

$$
\mathbb{E}\left[I_{p}(f) I_{q}(g)\right]=p ! \mathbf{1}_{\{p=q\}}\langle\tilde{f}, \tilde{g}\rangle_{\mathcal{H}^{\otimes p}},
$$

for any $f \in \mathcal{H}^{\otimes p}$ and $g \in \mathcal{H}^{\otimes q}$. We have the following product formula: For $f \in \mathcal{H}^{\odot p}$ and $g \in \mathcal{H}^{\odot q}$,

$$
I_{p}(f) I_{q}(g)=\sum_{r=0}^{p \wedge q} r !\left(\begin{array}{l}
p \\
r
\end{array}\right)\left(\begin{array}{l}
q \\
r
\end{array}\right) I_{p+q-2 r}\left(f \otimes_{r} g\right),
$$

where $f \otimes_{r} g$ is the $r$-contraction between $f$ and $g$, which is an element in $\mathcal{H}^{\otimes(p+q-2 r)}$ defined as follows. Fix an orthonormal basis $\left\{e_{i}, i \in \mathcal{O}\right\}$ of $\mathcal{H}$. Then, for $1 \leq r \leq p \wedge q$,

$$
\begin{aligned}
f \otimes_{r} g:= & \sum_{i_{1}, \ldots, i_{p}, j_{1}, \ldots, j_{q} \in \mathcal{O}}\left\langle f, e_{i_{1}} \otimes \cdots \otimes e_{i_{p}}\right\rangle_{\mathcal{H} \otimes p}\left\langle g, e_{j_{1}} \otimes \cdots \otimes e_{j_{q}}\right\rangle_{\mathcal{H} \otimes p} \\
& \times \mathbf{1}_{\left\{i_{k}=j_{k}, \forall k=1, \ldots, r\right\}} e_{i_{r+1}} \otimes \cdots \otimes e_{i_{p}} \otimes e_{j_{r+1}} \otimes \cdots \otimes e_{j_{q}} .
\end{aligned}
$$


In the particular case when $f, g$ are real-valued functions, we can write

$$
\begin{aligned}
(f & \left.\otimes_{r} g\right)\left(\boldsymbol{t}_{\boldsymbol{p}-\boldsymbol{r}}, \boldsymbol{x}_{\boldsymbol{p}-\boldsymbol{r}}, \boldsymbol{t}_{\boldsymbol{q}-\boldsymbol{r}}^{\prime}, \boldsymbol{x}_{\boldsymbol{q}-\boldsymbol{r}}^{\prime}\right) \\
& =\int_{\mathbb{R}_{+}^{2 r} \times \mathbb{R}^{2 r d}} d \boldsymbol{s}_{\boldsymbol{r}} d \boldsymbol{s}_{\boldsymbol{r}}^{\prime} d \boldsymbol{y}_{\boldsymbol{r}} d \boldsymbol{y}_{\boldsymbol{r}}^{\prime}\left(\prod_{j=1}^{r} \gamma_{0}\left(s_{j}-s_{j}^{\prime}\right) \gamma\left(y_{j}-y_{j}^{\prime}\right)\right) \\
& \times f\left(\boldsymbol{s}_{\boldsymbol{r}}, \boldsymbol{t}_{\boldsymbol{p}-\boldsymbol{r}}, \boldsymbol{y}_{\boldsymbol{r}}, \boldsymbol{x}_{\boldsymbol{p}-\boldsymbol{r}}\right) g\left(\boldsymbol{s}_{\boldsymbol{r}}^{\prime}, \boldsymbol{t}_{\boldsymbol{q}-\boldsymbol{r}}^{\prime}, \boldsymbol{y}_{\boldsymbol{r}}^{\prime}, \boldsymbol{x}_{\boldsymbol{q}-\boldsymbol{r}}^{\prime}\right),
\end{aligned}
$$

provided the above integral exists. For $F \in \mathbb{D}^{m, 2}$ with the representation (2.7) and $m \geq 1$, we have

$$
D_{\bullet}^{m} F=\sum_{n \geq m} \frac{n !}{(n-m) !} I_{n-m}\left(f_{n}(\bullet, *)\right) \text { with convergence in } L^{2}\left(\Omega ; \mathcal{H}^{\otimes m}\right),
$$

where $I_{n-m}\left(f_{n}(\bullet, *)\right)$ is understood as the $(n-m)$ th multiple integral of $f_{n}(\bullet, *) \in$ $\mathcal{H}^{\otimes(n-m)}$ for fixed $\bullet$. We can write

$$
D_{s_{m}, y_{m}}^{m} F=\sum_{n \geq m} \frac{n !}{(n-m) !} I_{n-m}\left(f_{n}\left(\boldsymbol{s}_{\boldsymbol{m}}, \boldsymbol{y}_{\boldsymbol{m}} ; *\right)\right)
$$

whenever the above series makes sense and converges in $L^{2}(\Omega)$. With the decomposition (2.11) in mind, we have the following Gaussian Poincaré inequality: For $F \in \mathbb{D}^{1,2}$, it holds that

$$
\operatorname{Var}(F) \leq \mathbb{E}\left[\|D F\|_{\mathcal{H}}^{2}\right]
$$

In fact, if $F$ has the representation (2.7), then

$$
\operatorname{Var}(F)=\sum_{n \geq 1} n !\left\|f_{n}\right\|_{\mathcal{H}^{\otimes n}}^{2} \quad \text { and } \quad \mathbb{E}\left[\|D F\|_{\mathcal{H}}^{2}\right]=\sum_{n \geq 1} n n !\left\|f_{n}\right\|_{\mathcal{H}^{\otimes n}}^{2},
$$

which gives us (2.12) and, moreover, indicates that the equality in (2.12) holds only when $F \in \mathbb{C}_{0}^{W} \oplus \mathbb{C}_{1}^{W}$, that is, only when $F$ is a real Gaussian random variable.

Now let us mention the particular case when the Gaussian noise is white in time, which is used in the reduction step in Sect. 3.2. First, let us denote

$$
\mathcal{H}_{0}:=L^{2}\left(\mathbb{R}_{+} ; \mathcal{P}_{0}\right)
$$

and point out that the following inequality reduces many calculations to the case of the white noise in time. For any nonnegative function $f \in \mathcal{H}_{0}^{\otimes n}$ that vanishes outside $\left([0, t] \times \mathbb{R}^{d}\right)^{n}$,

$$
\|f\|_{\mathcal{H}^{\otimes n}}^{2} \leq \Gamma_{t}^{n}\|f\|_{\mathcal{H}_{0}^{\otimes n}}^{2}
$$


where $^{7}$

$$
\Gamma_{t}=2 \int_{0}^{t} \gamma_{0}(s) d s \quad \text { and } \quad\|f\|_{\mathcal{H}_{0}^{\otimes n}}^{2}=\int_{[0, t]^{n}}\left\|f\left(t_{1}, \cdot, \ldots, t_{n}, \cdot\right)\right\|_{\mathcal{P}_{0}^{\otimes n}}^{2} d t_{1} \cdots d t_{n}
$$

whenever no ambiguity arises, we write $\|f\|_{0}:=\|f\|_{\mathcal{P}_{0}^{\otimes n}}$ so that $\|f\|_{\mathcal{H}_{0}^{\otimes n}}^{2}=$ $\int_{[0, t]^{n}}\left\|f\left(\boldsymbol{t}_{\boldsymbol{n}}, \bullet\right)\right\|_{0}^{2} d \boldsymbol{t}_{\boldsymbol{n}}$.

Let $\dot{\mathfrak{X}}$ denote the Gaussian noise that is white in time and has the same spatial correlation as $W$. More precisely, $\left\{\mathfrak{X}(f): f \in \mathcal{H}_{0}\right\}$ is a centered Gaussian family with covariance

$$
\mathbb{E}[\mathfrak{X}(f) \mathfrak{X}(g)]=\langle f, g\rangle_{\mathcal{H}_{0}}, \quad \text { for any } f, g \in \mathcal{H}_{0} \text {. }
$$

Denote by $I_{p}^{\mathfrak{X}}$ the $p$-th multiple stochastic integral with respect to $\mathfrak{X}$. The product formula (2.9) still holds with $W$ replaced by the noise $\mathfrak{X}$. Moreover, if $f \in \mathcal{H}^{\otimes p}$ and $g \in \mathcal{H}^{\otimes q}$ have disjoint temporal supports, ${ }^{8}$ then we have $f \otimes_{r} g=0$ for $r=$ $1, \ldots, p \wedge q$ and the product formula (2.9) reduces to

$$
I_{p}^{\mathfrak{X}}(f) I_{q}^{\mathfrak{X}}(g)=I_{p+q}^{\mathfrak{X}}(f \otimes g) .
$$

In this case, the random variables $I_{p}^{\mathfrak{X}}(f)$ and $I_{q}^{\mathfrak{X}}(g)$ are independent by the ÜstünelZakai-Kallenberg criterion (see Exercise 5.4 .8 of [25]) and note that we do not need to assume $f, g$ to be symmetric in (2.14).

Now let us introduce the Ornstein-Uhlenbeck operator $L$ that can be defined as follows. We say that $F$ belongs to the $\operatorname{Dom}(L)$ if $F \in \mathbb{D}^{1,2}$ and $D F \in \operatorname{Dom}(\delta)$; in this case, we let $L F=-\delta D F$. For $F \in L^{2}(\Omega)$ of the form (2.7), $F \in \operatorname{Dom}(L)$ if and only if $\sum_{n \geq 1} n^{2} n !\left\|f_{n}\right\|_{\mathcal{H}^{\otimes n}}^{2}<\infty$. In this case, we have $L F=\sum_{n \geq 1}-n I_{n}\left(f_{n}\right)$. Using the chaos expansion, we can also define the Ornstein-Uhlenbeck semigroup $\left\{P_{t}=e^{t L}, t \in \mathbb{R}_{+}\right\}$and the pseudo-inverse $L^{-1}$ of the Ornstein-Uhlenbeck operator $L$ as follows. For $F \in L^{2}(\Omega)$ having the chaos expansion (2.7),

$$
P_{t} F:=\sum_{n \geq 0} e^{-n t} I_{n}\left(f_{n}\right) \text { and } L^{-1} F=\sum_{n \geq 1}-\frac{1}{n} I_{n}\left(f_{n}\right) .
$$

$\overline{7}$ For the sake of completeness, we sketch a proof of (2.13) here: Given such a function $f \in \mathcal{H}_{0}^{\otimes n}$,

$$
\begin{aligned}
\|f\|_{\mathcal{H}}^{2 \otimes n} & =\int_{[0, t]^{2 n}} d \boldsymbol{s}_{\boldsymbol{n}} d \boldsymbol{t}_{\boldsymbol{n}}\left\langle f\left(\boldsymbol{s}_{\boldsymbol{n}}, \bullet\right),\left.f\left(\boldsymbol{t}_{\boldsymbol{n}}, \bullet\right)\right|_{\mathcal{P}_{0} \otimes n} \prod_{j=1}^{n} \gamma_{0}\left(s_{j}-t_{j}\right)\right. \\
& \leq \int_{[0, t]^{2 n}} d \boldsymbol{s}_{\boldsymbol{n}} d \boldsymbol{t}_{\boldsymbol{n}} \frac{1}{2}\left(\left\|f\left(\boldsymbol{s}_{\boldsymbol{n}}, \bullet\right)\right\|_{\mathcal{P}_{0}^{\otimes n}}^{2}+\left\|f\left(\boldsymbol{t}_{\boldsymbol{n}}, \bullet\right)\right\|_{\mathcal{P}_{0}^{\otimes n}}^{2}\right) \prod_{j=1}^{n} \gamma_{0}\left(s_{j}-t_{j}\right) \leq \Gamma_{t}^{n}\|f\|_{\mathcal{H}_{0}^{\otimes n}}^{2} .
\end{aligned}
$$

${ }^{8}$ This means $f=0$ outside $\left(J \times \mathbb{R}^{d}\right)^{p}$ and $g=0$ outside $\left(J^{c} \times \mathbb{R}^{d}\right)^{q}$ for some set $J \subset \mathbb{R}_{+}$. We will apply this formula to functions $f=f_{t, x, j}^{(j)}(r, z ; \bullet)$ and $g=f_{r, z, n-j}$ given in Sect. 3.1, in which case $J=(r, t)$. 
Observe that for any centered random variable $F \in L^{2}(\Omega, \sigma\{W\}, \mathbb{P}), L L^{-1} F=F$ and for any $G \in \operatorname{Dom}(L), L^{-1} L G=G-\mathbb{E}[G]$. The above expression and the modified isometry property (2.8) give us the contraction property of $P_{t}$ on $L^{2}(\Omega)$, that is, for $F \in L^{2}(\Omega, \sigma\{W\}, \mathbb{P}),\left\|P_{t} F\right\|_{2} \leq\|F\|_{2}$. Moreover, $P_{t}$ is a contraction operator on $L^{q}(\Omega)$ for any $q \in[1, \infty)$; see [25, Proposition 2.8.6].

Finally, let us recall Nelson's hypercontractivity property of the OrnsteinUhlenbeck semigroup: For $F \in L^{q}(\Omega, \sigma\{W\}, \mathbb{P})$ with $q \in(1, \infty)$, it holds for each $t \geq 0$ that $\left\|P_{t} F\right\|_{q_{t}} \leq\|F\|_{q}$ with $q_{t}=1+(q-1) e^{2 t}$. In this paper, we need one of its consequences - a moment inequality comparing $L^{q}(\Omega)$-norms on a fixed chaos:

$$
\text { If } F \in \mathbb{C}_{n}^{W} \text { and } p \in[2, \infty) \text {, then }\|F\|_{p} \leq(p-1)^{n / 2}\|F\|_{2} \text {; }
$$

see e.g. [25, Corollary 2.8.14].

\subsection{Inequalities}

Let us first present a few inequalities, which will be used in Sect. 3.

Lemma 2.1 Fix an integer $d \geq 1$. Suppose that either one of the following conditions hold:

(a) $\gamma \in L^{\ell}\left(\mathbb{R}^{d}\right)$ forsome $\in(1, \infty)$ (b) $\gamma(x)=|x|^{-\beta}$ forsome $\beta \in(0, d)$.

Define

$$
q= \begin{cases}\ell /(2 \ell-1) & \text { in case }(a) \\ d /(2 d-\beta) & \text { in case }(b)\end{cases}
$$

Then, for any $f, g \in L^{2 q}\left(\mathbb{R}^{d}\right)$,

$$
\int_{\mathbb{R}^{d}} \int_{\mathbb{R}^{d}} f(x) g(y) \gamma(x-y) d x d y \leq C_{\gamma}\|f\|_{L^{2 q}\left(\mathbb{R}^{d}\right)}\|g\|_{L^{2 q}\left(\mathbb{R}^{d}\right)},
$$

where $C_{\gamma}=\|\gamma\|_{L^{\ell}\left(\mathbb{R}^{d}\right)}$ in case (a), and $C_{\gamma}=C_{d, \beta}$ is the constant (depending on $d, \beta$ ) that appears in the Hardy-Littlewood-Sobolev inequality (2.16) below, in case (b).

Proof In the case $d=2$, this result was essentially proved on page 15 of [35] in case (a), and on page 6 of [4] in case (b). We reproduce the arguments here for the sake of completeness.

In case (a), we apply Hölder's inequality and Young's convolution inequality:

$$
\begin{aligned}
& \int_{\mathbb{R}^{d}} f(x)(g * \gamma)(x) d x \leq\|f\|_{L^{\frac{2 \ell}{2 \ell-1}\left(\mathbb{R}^{d}\right)}}\|g * \gamma\|_{L^{2 \ell}\left(\mathbb{R}^{d}\right)} \\
& \leq\|f\|_{L^{\frac{2 \ell}{2 \ell-1}\left(\mathbb{R}^{d}\right)}}\|g\|_{L^{2 \ell-1}\left(\mathbb{R}^{d}\right)}\|\gamma\|_{L^{\ell}\left(\mathbb{R}^{d}\right)} .
\end{aligned}
$$


In case (b), we apply Hölder's inequality and Hardy-Littlewood-Sobolev inequality:

$$
\begin{aligned}
& \int_{\mathbb{R}^{d}} f(x)(g * \gamma)(x) d x \leq\|f\|_{L^{\frac{2 d}{2 d-\beta}\left(\mathbb{R}^{d}\right)}}\|g * \gamma\|_{L^{2 d / \beta}\left(\mathbb{R}^{d}\right)} \\
& \quad \leq C_{d, \beta}\|f\|_{L^{\frac{2 d}{2 d-\beta}}\left(\mathbb{R}^{d}\right)}\|g\|_{L^{\frac{2 d}{2 d-\beta}}\left(\mathbb{R}^{d}\right)} \cdot
\end{aligned}
$$

This concludes the proof.

To deal with case (c) in (H1), we need the following modification of Lemma 2.1.

Lemma 2.2 Suppose that $\gamma\left(x_{1}, \ldots, x_{d}\right)=\prod_{i=1}^{d} \gamma_{i}\left(x_{i}\right)$, where for each $i \in$ $\{1, \ldots, d\}$,

$(M 1) \gamma_{i} \in L^{\ell_{i}}(\mathbb{R})$ for some $\ell_{i} \in(1, \infty)$ or $(M 2) \gamma_{i}(x)=|x|^{-\beta_{i}}$ for some $\beta_{i} \in(0,1)$

Let $q_{i}=\ell_{i} /\left(2 \ell_{i}-1\right)$ in case (M1) and $q_{i}=1 /\left(2-\beta_{i}\right)$ in case (M2). Let $q=$ $\max \left\{q_{i}: i=1, \ldots, d\right\}$.

If $f, g \in L^{2 q}\left(\mathbb{R}^{d}\right)$ satisfy $f(x)=g(x)=0$ for $x \notin \prod_{i=1}^{d}\left[a_{i}, b_{i}\right]$ for some real numbers $a_{i}<b_{i},{ }^{9}$ then

$$
\int_{\mathbb{R}^{d}} \int_{\mathbb{R}^{d}} f(x) g(y) \gamma(x-y) d x d y \leq \Lambda^{v} C_{\gamma}\|f\|_{L^{2 q}\left(\mathbb{R}^{d}\right)}\|g\|_{L^{2 q}\left(\mathbb{R}^{d}\right)},
$$

with $\Lambda=\max \left\{b_{i}-a_{i} ; i=1, \ldots, d\right\}, C_{\gamma}=\prod_{i=1}^{d} C_{\gamma_{i}}$ and $v=\sum_{i=1}^{d}\left(q_{i}^{-1}-q^{-1}\right)$. In particular, when $q_{i}=q$ for all $i \in\{1, \ldots, d\}$, we have

$$
\int_{\mathbb{R}^{d}} \int_{\mathbb{R}^{d}} f(x) g(y) \gamma(x-y) d x d y \leq C_{\gamma}\|f\|_{L^{2 q}\left(\mathbb{R}^{d}\right)}\|g\|_{L^{2 q}\left(\mathbb{R}^{d}\right)} .
$$

The constants $C_{\gamma_{i}}$ are defined as in Lemma 2.1 .

Proof By Lemma 2.1, inequality (2.17) holds for $d=1$ with $v=0$. Now let us consider $d \geq 2$ and prove inequality (2.17) by induction. Suppose (2.17) holds for $d \leq k-1(k \geq 2)$. We use the notation $x=\left(x_{1}, \ldots, x_{k}\right)=: x_{k}$.

Without loss of any generality we assume $q_{1} \geq q_{2} \geq \cdots \geq q_{k}$, so that $q=q_{1}$. Applying the initial step $(d=1)$ yields

$$
\begin{aligned}
& \int_{\mathbb{R}^{2 k}} d \boldsymbol{x}_{\boldsymbol{k}} d \boldsymbol{y}_{\boldsymbol{k}} f\left(\boldsymbol{x}_{\boldsymbol{k}}\right) g\left(\boldsymbol{y}_{\boldsymbol{k}}\right) \prod_{i=1}^{k} \gamma_{i}\left(x_{i}-y_{i}\right) \\
& \quad \leq C_{\gamma_{k}} \int_{\mathbb{R}^{2(k-1)}} d \boldsymbol{x}_{\boldsymbol{k}-\mathbf{1}} d \boldsymbol{y}_{\boldsymbol{k}-\mathbf{1}}\left\|f\left(\boldsymbol{x}_{\boldsymbol{k}-\mathbf{1}}, \bullet\right)\right\|_{L^{2 q_{k}(\mathbb{R})} \| g\left(\boldsymbol{y}_{\boldsymbol{k}-\mathbf{1}}, \bullet\right)} \|_{L^{2 q_{k}(\mathbb{R})}} \prod_{i=1}^{k-1} \gamma_{i}\left(x_{i}-y_{i}\right) .
\end{aligned}
$$

\footnotetext{
9 We can apply this lemma to the function $y \in \mathbb{R}^{2} \mapsto G_{t-s}(x-y)$ whose support is contained in $\left\{y \in \mathbb{R}^{2} ;|x-y|<t-s\right\}$, so we can choose $\Lambda=2 t-2 s$.
} 
By the induction hypothesis, we can bound the right-hand side of (2.18) by

$$
\begin{aligned}
& \left(\prod_{i=1}^{k} C_{\gamma_{i}}\right) \Lambda^{v^{*}}\left(\int_{\mathbb{R}^{k-1}}\left\|f\left(\boldsymbol{x}_{\boldsymbol{k}-\mathbf{1}}, \bullet\right)\right\|_{L^{2 q_{k}(\mathbb{R})}}^{2 q} d \boldsymbol{x}_{\boldsymbol{k}-\mathbf{1}}\right)^{\frac{1}{2 q}} \\
& \quad \times\left(\int_{\mathbb{R}^{k-1}}\left\|g\left(\boldsymbol{y}_{\boldsymbol{k}-\mathbf{1}}, \bullet\right)\right\|_{L^{2 q_{k}(\mathbb{R})}}^{2 q} d \boldsymbol{y}_{\boldsymbol{k}-\mathbf{1}}\right)^{\frac{1}{2 q}},
\end{aligned}
$$

with $v^{*}=\sum_{i=1}^{k-1}\left(q_{i}^{-1}-q^{-1}\right)$. By Hölder's inequality,

$$
\begin{aligned}
& \left(\int_{\mathbb{R}^{k-1}}\left\|f\left(\boldsymbol{x}_{\boldsymbol{k}-\mathbf{1}}, \bullet\right)\right\|_{L^{2 q_{k}(\mathbb{R})}}^{2 q} d \boldsymbol{x}_{\boldsymbol{k}-\mathbf{1}}\right)^{\frac{1}{2 q}} \\
& \quad=\left(\int_{\mathbb{R}^{k-1}}\left[\int_{a_{k}}^{b_{k}}\left|f\left(\boldsymbol{x}_{\boldsymbol{k}-\mathbf{1}}, x_{k}\right)\right|^{2 q_{k}} d x_{k}\right]^{\frac{2 q}{2 q_{k}}} d \boldsymbol{x}_{\boldsymbol{k}-\mathbf{1}}\right)^{\frac{1}{2 q}} \\
& \leq \Lambda^{\frac{1}{2 q_{k}}-\frac{1}{2 q}}\left(\int_{\mathbb{R}^{k-1}} \int_{a_{k}}^{b_{k}}\left|f\left(\boldsymbol{x}_{\boldsymbol{k}-\mathbf{1}}, x_{k}\right)\right|^{2 q} d x_{k} d \boldsymbol{x}_{\boldsymbol{k}-\mathbf{1}}\right)^{\frac{1}{2 q}}
\end{aligned}
$$

A similar inequality holds for $g$. Since $v^{*}+\left(q_{k}^{-1}-q^{-1}\right)=\sum_{i=1}^{k}\left(q_{i}^{-1}-q^{-1}\right)$, inequality (2.17) holds for $d=k$.

We will need the following generalization of Lemmas 2.1 and 2.2.

Lemma 2.3 (1) Under the conditions of Lemma 2.1, for any $f, g \in L^{2 q}\left(\mathbb{R}^{m d}\right)$

$$
\int_{\mathbb{R}^{2 m d}} f\left(\boldsymbol{x}_{\boldsymbol{m}}\right) g\left(\boldsymbol{y}_{\boldsymbol{m}}\right) \prod_{j=1}^{m} \gamma\left(x_{j}-y_{j}\right) d \boldsymbol{x}_{\boldsymbol{m}} d \boldsymbol{y}_{\boldsymbol{m}} \leq C_{\gamma}^{m}\|f\|_{L^{2 q}\left(\mathbb{R}^{m d}\right)}\|g\|_{L^{2 q}\left(\mathbb{R}^{m d}\right)},
$$

where $C_{\gamma}$ is the same constant as in Lemma 2.1. Here $\boldsymbol{x}_{\boldsymbol{m}}=\left(x_{1}, \ldots, x_{m}\right)$ with $x_{i} \in \mathbb{R}^{d}$.

(2) Let $\gamma, C_{\gamma}$ and $q$ be given as in Lemma 2.2. If $f, g \in L^{2 q}\left(\mathbb{R}^{m d}\right)$ satisfy $f\left(\boldsymbol{x}_{\boldsymbol{m} \boldsymbol{d}}\right)=$ $g\left(\boldsymbol{x}_{\boldsymbol{m} \boldsymbol{d}}\right)=0$ for $\boldsymbol{x}_{\boldsymbol{m} \boldsymbol{d}} \notin \prod_{i=1}^{m d}\left[a_{i}, b_{i}\right]$ for some real numbers $a_{i}<b_{i}$, then inequality (2.19) holds with $C_{\gamma}$ replaced by $\Lambda^{\nu} C_{\gamma}$, where $\Lambda=\max \left\{b_{i}-a_{i}: i=1, \ldots, m d\right\}$ and $v=\sum_{i=1}^{d}\left(q_{i}^{-1}-q^{-1}\right)$. Here $\boldsymbol{x}_{\boldsymbol{m} \boldsymbol{d}}=\left(x_{1}, \ldots, x_{m d}\right)$ with $x_{i} \in \mathbb{R}$.

Proof The proof will be done by induction on $m$ simultaneously for both cases (1) and (2). Let $C=C_{\gamma}$ in case (1) and $C=\Lambda^{v} C_{\gamma}$ in case (2). The results are true for $m=1$ by Lemmas 2.1 and 2.2. Assume that the results hold for $m-1$. Applying the inequality for $m=1$ yields 


$$
\begin{aligned}
& \int_{\mathbb{R}^{2 d m}} f\left(\boldsymbol{x}_{\boldsymbol{m}}\right) g\left(\boldsymbol{y}_{\boldsymbol{m}}\right) \prod_{j=1}^{m} \gamma\left(x_{j}-y_{j}\right) d \boldsymbol{x}_{\boldsymbol{m}} d \boldsymbol{y}_{\boldsymbol{m}} \\
& \leq C \int_{\mathbb{R}^{2 d(m-1)}}\left\|f\left(\boldsymbol{x}_{\boldsymbol{m}-\mathbf{1}}, \bullet\right)\right\|_{L^{2 q}\left(\mathbb{R}^{d}\right)}\left\|g\left(\boldsymbol{y}_{\boldsymbol{m}-\mathbf{1}}, \bullet\right)\right\|_{L^{2 q}\left(\mathbb{R}^{d}\right)} \\
& \quad \times \prod_{j=1}^{m-1} \gamma\left(x_{j}-y_{j}\right) d \boldsymbol{x}_{\boldsymbol{m}-\mathbf{1}} d \boldsymbol{y}_{\boldsymbol{m}-\mathbf{1}} .
\end{aligned}
$$

By the induction hypothesis, the latter term can be bounded by

$$
\begin{aligned}
& C^{m}\left(\int_{\mathbb{R}^{d(m-1)}}\left\|f\left(\boldsymbol{x}_{\boldsymbol{m}-\mathbf{1}}, \bullet\right)\right\|_{L^{2 q\left(\mathbb{R}^{d}\right)}}^{2 q} d \boldsymbol{x}_{\boldsymbol{m}-\mathbf{1}}\right)^{\frac{1}{2 q}} \\
& \quad \times\left(\int_{\mathbb{R}^{d(m-1)}}\left\|g\left(\boldsymbol{x}_{\boldsymbol{m}-\mathbf{1}}, \bullet\right)\right\|_{L^{2 q\left(\mathbb{R}^{d}\right)}}^{2 q} d \boldsymbol{x}_{\boldsymbol{m}-\mathbf{1}}\right)^{\frac{1}{2 q}},
\end{aligned}
$$

which completes the proof.

Let us return to the three cases of Hypothesis (H1). Lemma 2.1 indicates that $L^{2 q}\left(\mathbb{R}^{2}\right)$ is continuously embedded into $\mathcal{P}_{0}$, with $q \in(1 / 2,1)$ given by

$$
q= \begin{cases}\ell /(2 \ell-1) & \text { in case }(\mathrm{a}) \\ 2 /(4-\beta) & \text { in case }(\mathrm{b})\end{cases}
$$

Recall that $\mathcal{P}_{0}$ has been defined at the beginning of Sect. 2.1. Moreover, for any $f, g \in L^{2 q}\left(\mathbb{R}^{2}\right)$,

$$
\int_{\mathbb{R}^{4}}|f(x) g(x)| \gamma(x-y) d x d y \leq D_{\gamma}\|f\|_{L^{2 q\left(\mathbb{R}^{2}\right)}}\|g\|_{L^{2 q}\left(\mathbb{R}^{2}\right)},
$$

where

$$
D_{\gamma}= \begin{cases}\|\gamma\|_{L^{\ell}\left(\mathbb{R}^{2}\right)} & \text { in case (a) } \\ C_{2, \beta} & \text { in case (b) }\end{cases}
$$

For case (c) of Hypothesis (H1), we consider three sub-cases:

(i) $\gamma_{i} \in L^{\ell_{i}}(\mathbb{R})$ for some $\ell_{i}>1, i=1,2$;

(ii) $\gamma_{i}\left(x_{i}\right)=\left|x_{i}\right|^{-\beta_{i}}$ for some $\beta_{i} \in(0,1), i=1,2$;

(iii) $\gamma_{1} \in L^{\ell}(\mathbb{R})$ for some $\ell \in(1, \infty)$ and $\gamma_{2}\left(x_{2}\right)=\left|x_{2}\right|^{-\beta}$ for some $\beta \in(0,1)$. 
Lemma 2.2 implies that, for any $f, g \in L^{2 q}\left(\mathbb{R}^{2}\right)$ with

$$
q= \begin{cases}\max \left\{\ell_{i} /\left(2 \ell_{i}-1\right): i=1,2\right\} & \text { in case (i) } \\ \max \left\{1 /\left(2-\beta_{i}\right): i=1,2\right\} & \text { in case (ii) } \\ \max \{\ell /(2 \ell-1), 1 /(2-\beta)\} & \text { in case (iii) }\end{cases}
$$

such that $f, g$ vanish outside a box with side lengths bounded by $\Lambda$, then inequality (2.21) still holds with

$$
D_{\gamma}= \begin{cases}\left\|\gamma_{1}\right\|_{L^{\ell_{1}}(\mathbb{R})}\left\|\gamma_{2}\right\|_{L^{\ell_{2}(\mathbb{R})}} \Lambda^{\left|\frac{1}{\ell_{1}}-\frac{1}{\ell_{2}}\right|} & \text { in case (i) } \\ C_{1, \beta_{1}} C_{1, \beta_{2}} \Lambda^{\left|\beta_{1}-\beta_{2}\right|} & \text { in case (ii) } \\ C_{1, \beta}\left\|\gamma_{1}\right\|_{L^{\ell}(\mathbb{R})} \Lambda^{\left|\frac{1}{\ell}-\beta\right|} & \text { in case (iii) }\end{cases}
$$

where the constants $C_{1, \beta_{i}}$ are given as in Lemma 2.1.

From Lemma 2.3, we deduce that in cases (a) and (b),

$$
\|f\|_{\mathcal{H}_{0}^{\otimes n}}^{2} \leq D_{\gamma}^{n} \int_{[0, t]^{n}}\left\|f\left(\boldsymbol{t}_{\boldsymbol{n}}, \bullet\right)\right\|_{L^{2 q}\left(\mathbb{R}^{2 n}\right)}^{2} d \boldsymbol{t}_{\boldsymbol{n}},
$$

for any measurable function $f:\left(\mathbb{R}_{+} \times \mathbb{R}^{2}\right)^{n} \rightarrow \mathbb{R}$ such that $f$ vanishes outside $\left([0, t] \times \mathbb{R}^{2}\right)^{n}$; in case (c), inequality (2.25) holds true for any measurable function $f:\left(\mathbb{R}_{+} \times \mathbb{R}^{2}\right)^{n} \rightarrow \mathbb{R}$ such that

$$
f\left(t_{1}, x_{1}, \ldots, t_{n}, x_{n}\right)=f\left(\boldsymbol{t}_{\boldsymbol{n}}, \boldsymbol{x}_{\boldsymbol{n}}\right)=0 \text { for } \boldsymbol{t}_{\boldsymbol{n}} \notin[0, t]^{n} \text { and } \boldsymbol{x}_{\boldsymbol{n}} \notin \prod_{i=1}^{2 n}\left[a_{i}, b_{i}\right]
$$

with $\Lambda:=\max \left\{b_{i}-a_{i}: i=1, \ldots, 2 n\right\}<\infty$.

Let us present a few facts on the fundamental solution $G$. When $d=2$,

$$
\begin{aligned}
\left\|G_{t}\right\|_{L^{p}\left(\mathbb{R}^{2}\right)} & =\left(\frac{(2 \pi)^{1-p}}{2-p}\right)^{1 / p} t^{\frac{2}{p}-1} \text { for all } p \in(0,2) \\
G_{t}^{p}(x) & \leq(2 \pi t)^{q-p} G_{t}^{q}(x) \text { for all } p<q
\end{aligned}
$$

and

$$
\mathbf{1}_{\{|x|<t\}} \leq 2 \pi t G_{t}(x)
$$

We will use also the following estimate.

Lemma 2.4 (Lemma 4.3 of [4]) For any $q \in(1 / 2,1)$ and $d=2$,

$$
\int_{r}^{t}\left(G_{t-s}^{2 q} * G_{s-r}^{2 q}\right)^{1 / q}(z) d s \leq A_{q}(t-r)^{\frac{1}{q}-1} G_{t-r}^{2-\frac{1}{q}}(z),
$$

where $A_{q}>0$ is a constant depending on $q$. 
Finally, we record the expression of the Fourier transform of $G_{t}$ for $d \in\{1,2\}$ :

$$
\mathcal{F} G_{t}(\xi)=\int_{\mathbb{R}^{d}} e^{-i \xi \cdot x} G_{t}(x) d x=\frac{\sin (t|\xi|)}{|\xi|}=: \widehat{G}_{t}(\xi)
$$

Note that (see e.g. (3.4) of [3])

$$
\left|\widehat{G}_{t}(\xi)\right|^{2} \leq 2\left(t^{2} \vee 1\right) \frac{1}{1+|\xi|^{2}}
$$

In Sect. 4, we need the following two results.

Lemma 2.5 For $d \in\{1,2\}$, let $\gamma_{0}$ satisfy the assumption (i) on page 2 and let $\mu_{p}$ be a symmetric measure on $\left(\mathbb{R}^{d}\right)^{p}$, for some integer $p \geq 1$. Then, with $0<s \leq t$ and $\Delta_{p}(t)=\left\{\boldsymbol{s}_{\boldsymbol{p}} \in \mathbb{R}_{+}^{p}: t=s_{0}>s_{1}>\cdots>s_{p}>0\right\}$,

$$
\begin{aligned}
& \sum_{\sigma \in \mathfrak{S}_{p}} \int_{\Delta_{p}(t)} d \boldsymbol{s}_{\boldsymbol{p}} \int_{[0, s]^{p}} d \tilde{\boldsymbol{s}}_{\boldsymbol{p}} \mathbf{1}_{\left\{s>\tilde{s}_{\sigma(1)>\cdots>\tilde{s}_{\sigma(p)}>0}\right.}\left(\prod_{j=1}^{p} \gamma_{0}\left(s_{j}-\tilde{s}_{j}\right)\right) \int_{\mathbb{R}^{p d}} \mu_{p}\left(d \boldsymbol{\xi}_{\boldsymbol{p}}\right) \\
& \quad \times g\left(s_{1}, \xi_{1}, \ldots, s_{p}, \xi_{p}\right) g\left(\tilde{s}_{\sigma(1)}, \xi_{\sigma(1)}, \ldots, \tilde{s}_{\sigma(p)}, \xi_{\sigma(p)}\right) \\
& \quad \leq \Gamma_{t}^{p} \int_{\Delta_{p}(t)} d \boldsymbol{s}_{\boldsymbol{p}} \int_{\mathbb{R}^{p d}} \mu_{p}\left(d \boldsymbol{\xi}_{\boldsymbol{p}}\right) g\left(s_{1}, \xi_{1}, \ldots, s_{p}, \xi_{p}\right)^{2}, \text { with } \Gamma_{t}:=\int_{-t}^{t} \gamma_{0}(a) d a,
\end{aligned}
$$

for any measurable function $g:\left(\mathbb{R}_{+} \times \mathbb{R}^{d}\right)^{p} \rightarrow \mathbb{R}_{+}$for which the above integral is finite.

Proof After applying $|a b| \leq \frac{a^{2}+b^{2}}{2}$ and using the symmetry of $\mu_{p}$, we have that the left-hand side quantity is bounded by

$$
\begin{aligned}
& \frac{1}{2} \sum_{\sigma \in \mathfrak{S}_{p}} \int_{\Delta_{p}(t)} d \boldsymbol{s}_{\boldsymbol{p}} \int_{[0, s]^{p}} d \tilde{\boldsymbol{s}}_{\boldsymbol{p}} \mathbf{1}_{\left\{s>\tilde{s}_{\left.\sigma(1)>\cdots>\tilde{s}_{\sigma(p)>0}\right\}} h\left(\boldsymbol{s}_{\boldsymbol{p}}\right) \prod_{j=1}^{p} \gamma_{0}\left(s_{j}-\tilde{s}_{j}\right)\right.} \\
& \quad+\frac{1}{2} \sum_{\sigma \in \mathfrak{S}_{p}} \int_{\Delta_{p}(t)} d \boldsymbol{s}_{\boldsymbol{p}} \int_{[0, s]^{p}} d \tilde{\boldsymbol{s}}_{\boldsymbol{p}} \mathbf{1}_{\left\{s>\tilde{s}_{\sigma(1)>\cdots>\tilde{s}_{\sigma(p)}>0}\right.} h\left(\tilde{s}_{\sigma(1)}, \ldots, \tilde{s}_{\sigma(p)}\right) \prod_{j=1}^{p} \gamma_{0}\left(s_{j}-\tilde{s}_{j}\right)
\end{aligned}
$$

with

$$
h\left(s_{1}, \ldots, s_{p}\right):= \begin{cases}\int_{\mathbb{R} p d} \mu_{p}\left(d \boldsymbol{\xi}_{\boldsymbol{p}}\right) g\left(s_{1}, \xi_{1}, \ldots, s_{p}, \xi_{p}\right)^{2}, & \text { for } \boldsymbol{s}_{\boldsymbol{p}} \in \Delta_{p}(t) \\ 0, & \text { otherwise. }\end{cases}
$$


Putting $\mathcal{I}_{s}\left(s_{1}, \ldots, s_{p}\right):=\mathbf{1}_{\left\{s>s_{1}>\cdots>s_{p}>0\right\}}$ and letting $\widetilde{\mathcal{I}}_{s}\left(s_{1}, \ldots, s_{p}\right)$ be its canonical symmetrization (so that $\left|\widetilde{\mathcal{I}}_{s}\right| \leq(p !)^{-1}$ ), we can rewrite the term in (2.31) as

$$
\begin{aligned}
& \frac{p !}{2} \int_{\Delta_{p}(t)} \int_{[0, s]^{p}} d \boldsymbol{s}_{\boldsymbol{p}} d \tilde{\boldsymbol{s}}_{\boldsymbol{p}} h\left(\boldsymbol{s}_{\boldsymbol{p}}\right) \widetilde{\mathcal{I}}_{s}\left(\tilde{\boldsymbol{s}}_{\boldsymbol{p}}\right) \prod_{j=1}^{p} \gamma_{0}\left(s_{j}-\tilde{s}_{j}\right) \\
& \quad \leq \frac{1}{2} \int_{\Delta_{p}(t)} \int_{[0, s]^{p}} d \boldsymbol{s}_{\boldsymbol{p}} d \tilde{\boldsymbol{s}}_{\boldsymbol{p}} h\left(\boldsymbol{s}_{\boldsymbol{p}}\right) \prod_{j=1}^{p} \gamma_{0}\left(s_{j}-\tilde{s}_{j}\right) \\
& \quad \leq \frac{1}{2} \Gamma_{t}^{p} \int_{\Delta_{p}(t)} d \boldsymbol{s}_{\boldsymbol{p}} h\left(\boldsymbol{s}_{\boldsymbol{p}}\right)
\end{aligned}
$$

using also the bound $\sup \left\{\int_{0}^{s} \gamma_{0}\left(r-r^{\prime}\right) d r^{\prime}: r \in[0, t]\right\} \leq \Gamma_{t}$. For the other term (2.32), we argue in the same way: With $\left(\mathcal{I}_{s} \cdot h\right)\left(s_{1}, \ldots, s_{p}\right)=\mathcal{I}_{s}\left(s_{1}, \ldots, s_{p}\right) h\left(s_{1}, \ldots, s_{p}\right)$, we rewrite the term $(2.32)$ as

$$
\begin{aligned}
& \frac{p !}{2} \int_{[0, t]^{p}} d \boldsymbol{s}_{\boldsymbol{p}} \int_{[0, s]^{p}} d \tilde{\boldsymbol{s}}_{\boldsymbol{p}} \mathcal{I}_{t}\left(\boldsymbol{s}_{\boldsymbol{p}}\right) \times \widetilde{\left(\mathcal{I}_{s} \cdot h\right)}\left(\tilde{\boldsymbol{s}}_{\boldsymbol{p}}\right) \prod_{j=1}^{p} \gamma_{0}\left(s_{j}-\tilde{s}_{j}\right)=\frac{p !}{2}\left\langle\mathcal{I}_{t}, \widetilde{\mathcal{I}_{s} \cdot h}\right\rangle_{\mathcal{H}^{\otimes p}} \\
& \quad=\frac{p !}{2}\left\langle\widetilde{\mathcal{I}}_{t}, \mathcal{I}_{s} \cdot h\right\rangle_{\mathcal{H}^{\otimes p}} \leq \frac{1}{2} \int_{[0, t]^{p}} d \boldsymbol{t}_{\boldsymbol{p}} \int_{\Delta_{p}(s)} h\left(\tilde{\boldsymbol{s}}_{\boldsymbol{p}}\right) \prod_{j=1}^{p} \gamma_{0}\left(s_{j}-\tilde{s}_{j}\right) \\
& \quad \leq \frac{1}{2} \Gamma_{t}^{p} \int_{\Delta_{p}(s)} d \boldsymbol{s}_{\boldsymbol{p}} h\left(\boldsymbol{s}_{\boldsymbol{p}}\right),
\end{aligned}
$$

since $h \geq 0$ and $\left|\widetilde{\mathcal{I}}_{t}\right| \leq(p !)^{-1}$. This concludes the proof.

Lemma 2.6 For $d \in\{1,2\}$ let $\gamma, \mu$ satisfy the assumption (ii) on page 2. Then, for any nonnegative function $h \in \mathcal{P}_{0} \cap L^{1}\left(\mathbb{R}^{d}\right)$,

$$
\sup _{z \in \mathbb{R}^{d}} \int_{\mathbb{R}^{d}} \mu(d \xi)|\mathcal{F} h(\xi+z)|^{2} \leq \int_{\mathbb{R}^{d}} \mu(d \xi)|\mathcal{F} h(\xi)|^{2}
$$

As a consequence, for any integer $p \geq 1$ and $w_{1}, \ldots, w_{p} \in[0, t]$,

$$
\sup _{\boldsymbol{w}_{\boldsymbol{p}} \in[0, t]^{p}} \sup _{z_{\boldsymbol{p}} \in \mathbb{R}^{d p}} \int_{\mathbb{R}^{d p}} \mu\left(d \boldsymbol{\xi}_{\boldsymbol{p}}\right) \prod_{j=1}^{p}\left|\widehat{G}_{w_{j}}\left(\xi_{j}+z_{j}\right)\right|^{2} \leq\left(2\left(t^{2} \vee 1\right) \int_{\mathbb{R}^{d}} \frac{\mu(d \xi)}{1+|\xi|^{2}}\right)^{p}
$$

Proof Since $h \geq 0$, using the fact that $\mathcal{F} h(\xi+z)=\mathcal{F}\left(e^{-i z \cdot h}\right)(\xi)$ together with $\left|e^{-i z(x+y)}\right|=1$, we get

$$
\int_{\mathbb{R}^{d}} \mu(d \xi)|\mathcal{F} h(\xi+z)|^{2}=\int_{\mathbb{R}^{2 d}} e^{-i z(x+y)} h(x) h(y) \gamma(x-y) d x d y
$$




$$
\leq \int_{\mathbb{R}^{2 d}} h(x) h(y) \gamma(x-y) d x d y
$$

which is exactly $\int_{\mathbb{R}^{d}} \mu(d \xi)|\mathcal{F} h(\xi)|^{2}$. In particular, by (2.30),

$$
\sup _{z \in \mathbb{R}^{d}} \int_{\mathbb{R}^{d}} \mu(d \xi)\left|\widehat{G}_{s}(\xi+z)\right|^{2} \leq \int_{\mathbb{R}^{d}} \mu(d \xi)\left|\widehat{G}_{s}(\xi)\right|^{2} \leq 2\left(s^{2} \vee 1\right) \int_{\mathbb{R}^{d}} \frac{\mu(d \xi)}{1+|\xi|^{2}}
$$

which is finite due to Dalang's condition (1.2). Applying this inequality several times yields

$$
\int_{\mathbb{R}^{d p}} \mu\left(d \boldsymbol{\xi}_{\boldsymbol{p}}\right) \prod_{j=1}^{p}\left|\widehat{G}_{w_{j}}\left(\xi_{j}+z_{j}\right)\right|^{2} \leq\left(2\left(t^{2} \vee 1\right) \int_{\mathbb{R}^{d}} \frac{\mu(d \xi)}{1+|\xi|^{2}}\right)^{p}
$$

which is a uniform bound over $\left(\boldsymbol{z}_{\boldsymbol{p}}, \boldsymbol{w}_{\boldsymbol{p}}\right) \in \mathbb{R}^{d p} \times[0, t]^{p}$.

\section{$3 L^{p}$ estimates for Malliavin derivatives}

This section is mainly devoted to the proof of Theorem 1.3. The proof will be done in several steps organized in Sects. 3.1, 3.2, 3.3, 3.4 and 3.5. In Sect. 3.6, we record a few consequences of Theorem 1.3 that will be used in the proof of Theorem 1.10 in Sect. 5.

\subsection{Step 1: Preliminaries}

Let us first introduce some handy notation. Recall that for $\boldsymbol{t}_{\boldsymbol{n}}:=\left(t_{1}, \ldots, t_{n}\right)$ and $x_{n}:=\left(x_{1}, \ldots, x_{n}\right)$, we defined in (1.8)

$$
f_{t, x, n}\left(\boldsymbol{t}_{\boldsymbol{n}}, \boldsymbol{x}_{\boldsymbol{n}}\right)=G_{t-t_{1}}\left(x-x_{1}\right) G_{t_{1}-t_{2}}\left(x_{1}-x_{2}\right) \cdots G_{t_{n-1}-t_{n}}\left(x_{n-1}-x_{n}\right),
$$

with the convention (1.6), and we denote by $\widetilde{f}_{t, x, n}$ the symmetrization of $f_{t, x, n}$; see (1.9). We treat the time-space variables $\left(t_{i}, x_{i}\right)$ as one coordinate and we write

$$
f_{t, x, n}\left(r, z ; \boldsymbol{t}_{\boldsymbol{n}-\mathbf{1}}, \boldsymbol{x}_{\boldsymbol{n}-1}\right):=f_{t, x, n}\left(r, z, t_{1}, x_{1}, \ldots, t_{n-1}, x_{n-1}\right)
$$

as in Notation A-(3). Recall that the solution $u(t, x)$ has the Wiener chaos expansion

$$
u(t, x)=1+\sum_{n=1}^{\infty} I_{n}\left(f_{t, x, n}\right)
$$

where the kernel $f_{t, x, n}$ is not symmetric and in this case, by definition, $I_{n}\left(f_{t, x, n}\right)=$ $I_{n}\left(\widetilde{f}_{t, x, n}\right)$. 
Our first goal is to show that, for any fixed $(r, z) \in[0, t] \times \mathbb{R}^{d}$ and for any $p \in$ $[2, \infty)$, the series

$$
\sum_{n \geq 1} n I_{n-1}\left(\widetilde{f}_{t, x, n}(r, z ; \bullet)\right)
$$

converges in $L^{p}(\Omega)$, and the sum, denoted by $D_{r, z} u(t, x)$, satisfies the $L^{p}$ estimates (1.11).

The first term of the series (3.1) is $\widetilde{f}_{t, x, 1}(r, z)=G_{t-r}(x-z)$. In general, for any $n \geq 1$,

$$
\widetilde{f}_{t, x, n}(r, z ; \bullet)=\frac{1}{n} \sum_{j=1}^{n} h_{t, x, n}^{(j)}(r, z ; \bullet),
$$

where $h_{t, x, n}^{(j)}(r, z ; \bullet)$ is the symmetrization of the function $\left(\boldsymbol{t}_{\boldsymbol{n}-\mathbf{1}}, \boldsymbol{x}_{\boldsymbol{n}-\mathbf{1}}\right) \rightarrow f_{t, x, n}^{(j)}$ $\left(r, z ; \boldsymbol{t}_{\boldsymbol{n}-1}, \boldsymbol{x}_{\boldsymbol{n}-1}\right)$, which is obtained from $f_{t, x, n}$ by placing $r$ on position $j$ among the time instants, and $z$ on position $j$ among the space points: With the convention (1.6),

$$
\begin{aligned}
& f_{t, x, n}^{(j)}\left(r, z ; \boldsymbol{t}_{\boldsymbol{n}-\mathbf{1}}, \boldsymbol{x}_{\boldsymbol{n}-1}\right) \\
& \quad=G_{t-t_{1}}\left(x-x_{1}\right) \cdots G_{t_{j-1}-r}\left(x_{j-1}-z\right) G_{r-t_{j}}\left(z-x_{j}\right) \cdots G_{t_{n-2}-t_{n-1}}\left(x_{n-2}-x_{n-1}\right) .
\end{aligned}
$$

That is,

$$
f_{t, x, n}^{(j)}(r, z ; \bullet)=f_{t, x, j}^{(j)}(r, z ; \bullet) \otimes f_{r, z, n-j},
$$

with $f_{r, z, 1}=1$. For example, $f_{t, x, 1}^{(1)}(r, z ; \bullet)=G_{t-r}(x-z)$ and $f_{t, x, n}^{(1)}\left(r, z ; \boldsymbol{t}_{\boldsymbol{n}-\mathbf{1}}, \boldsymbol{x}_{\boldsymbol{n}-\mathbf{1}}\right)$ $=G_{t-r}(x-z) f_{r, z, n-1}\left(\boldsymbol{t}_{\boldsymbol{n}-1}, \boldsymbol{x}_{\boldsymbol{n}-1}\right)$. By the definition of the symmetrization,

$$
\begin{aligned}
& h_{t, x, n}^{(j)}\left(r, z ; \boldsymbol{t}_{\boldsymbol{n}-1}, \boldsymbol{x}_{\boldsymbol{n}-1}\right) \\
& \quad=\frac{1}{(n-1) !} \sum_{\sigma \in \mathfrak{S}_{n-1}} f_{t, x, n}^{(j)}\left(r, z ; t_{\sigma(1)}, x_{\sigma(1)}, \ldots, t_{\sigma(n-1)}, x_{\sigma(n-1)}\right) .
\end{aligned}
$$

Similarly, for $\boldsymbol{s}_{\boldsymbol{m}} \in[0, t]^{m}$ and $\boldsymbol{y}_{\boldsymbol{m}} \in \mathbb{R}^{d m}$, and for any $p \in[2, \infty)$, we will show that

$$
D_{\boldsymbol{s}_{\boldsymbol{m}}, y_{\boldsymbol{m}}}^{m} u(t, x):=\sum_{n \geq m} \frac{n !}{(n-m) !} I_{n-m}\left(\widetilde{f}_{t, x, n}\left(\boldsymbol{s}_{\boldsymbol{m}}, \boldsymbol{y}_{\boldsymbol{m}} ; \bullet\right)\right)
$$

converges in $L^{p}(\Omega)$. Note that if the series (3.6) converges in $L^{p}(\Omega)$, we can see that almost surely, the function

$$
\left(\boldsymbol{s}_{\boldsymbol{m}}, \boldsymbol{y}_{\boldsymbol{m}}\right) \mapsto D_{\boldsymbol{s}_{\boldsymbol{m}}, y_{\boldsymbol{m}}}^{m} u(t, x)
$$


is symmetric, meaning that for any $\sigma \in \mathfrak{S}_{m}$,

$$
D_{s_{1}, y_{1}} D_{s_{2}, y_{2}} \cdots D_{s_{m}, y_{m}} u(t, x)=D_{s_{\sigma(1)}, y_{\sigma(1)}} D_{s_{\sigma(2)}, y_{\sigma(2)}} \cdots D_{s_{\sigma(m)}, y_{\sigma(m)}} u(t, x) .
$$

From now on, we assume $t>s_{1}>\ldots>s_{m}>0$ without losing any generality. Note that like (3.2), we can write

$$
\frac{n !}{(n-m) !} \widetilde{f}_{t, x, n}\left(\boldsymbol{s}_{\boldsymbol{m}}, \boldsymbol{y}_{\boldsymbol{m}} ; \bullet\right)=\sum_{\boldsymbol{i}_{\boldsymbol{m}} \in \Delta_{n, m}} h_{t, x, n}^{\left(\boldsymbol{i}_{\boldsymbol{m}}\right)}\left(\boldsymbol{s}_{\boldsymbol{m}}, \boldsymbol{y}_{\boldsymbol{m}} ; \bullet\right),
$$

where $\boldsymbol{i}_{\boldsymbol{m}} \in \Delta_{n, m}$ means $1 \leq i_{1}<i_{2}<\cdots<i_{m} \leq n$ and $h_{t, x, n}^{\left(\boldsymbol{i}_{\boldsymbol{m}}\right)}\left(\boldsymbol{s}_{\boldsymbol{m}}, \boldsymbol{y}_{\boldsymbol{m}} ; \bullet\right)$ is the symmetrization of the function $f_{t, x, n}^{\left(i_{m}\right)}\left(\boldsymbol{s}_{\boldsymbol{m}}, \boldsymbol{y}_{\boldsymbol{m}} ; \bullet\right)$ that is defined by

$$
\begin{aligned}
f_{t, x, n}^{\left(i_{\boldsymbol{m}}\right)}\left(\boldsymbol{s}_{\boldsymbol{m}}, \boldsymbol{y}_{\boldsymbol{m}} ; \bullet\right)= & f_{t, x, i_{1}}^{\left(i_{1}\right)}\left(s_{1}, y_{1} ; \bullet\right) \otimes f_{s_{1}, y_{1}, i_{2}-i_{1}}^{\left(i_{2}-i_{1}\right)}\left(s_{2}, y_{2} ; \bullet\right) \otimes \cdots \otimes \\
& f_{s_{m-1}, y_{m-1}, i_{m}-i_{m-1}}^{\left(i_{m}-i_{m-1}\right)}\left(s_{m}, y_{m} ; \bullet\right) \otimes f_{s_{m}, y_{m}, n-i_{m}},
\end{aligned}
$$

which is a generalization of (3.4).

\subsection{Step 2: Reduction to white noise in time}

Let $\dot{\mathfrak{X}}$ denote the Gaussian noise that is white in time and has the same spatial correlation as $W$ and let $\left\{\mathfrak{X}(f): f \in \mathcal{H}_{0}\right\}$ denote the resulting isonormal Gaussian process; see Sect. 2.1.

For any $p \in[2, \infty)$, we deduce from (3.6) and (3.7) that

$$
\begin{aligned}
\left\|D_{\boldsymbol{s}_{\boldsymbol{m}}, \boldsymbol{y}_{\boldsymbol{m}}}^{m} u(t, x)\right\|_{p} & \leq \sum_{n \geq m}\left\|I_{n-m}\left(\sum_{i_{\boldsymbol{m}} \in \Delta_{n, m}} h_{t, x, n}^{\left(\boldsymbol{i}_{\boldsymbol{m}}\right)}\left(\boldsymbol{s}_{\boldsymbol{m}}, \boldsymbol{y}_{\boldsymbol{m}} ; \bullet\right)\right)\right\|_{p} \text { by triangle inequality } \\
& \leq \sum_{n \geq m}(p-1)^{\frac{n-m}{2}}\left\|I_{n-m}\left(\sum_{\boldsymbol{i}_{\boldsymbol{m}} \in \Delta_{n, m}} h_{t, x, n}^{\left(\boldsymbol{i}_{\boldsymbol{m}}\right)}\left(\boldsymbol{s}_{\boldsymbol{m}}, \boldsymbol{y}_{\boldsymbol{m}} ; \bullet\right)\right)\right\|_{2} \text { by (2.15). }
\end{aligned}
$$

The function $\sum_{i_{\boldsymbol{m}} \in \Delta_{n, m}} h_{t, x, n}^{\left(\boldsymbol{i}_{\boldsymbol{m}}\right)}\left(\boldsymbol{s}_{\boldsymbol{m}}, \boldsymbol{y}_{\boldsymbol{m}} ; \bullet\right)$ vanishes outside $\left([0, t] \times \mathbb{R}^{d}\right)^{n-m}$, thus we deduce from (2.13) that

$$
\begin{aligned}
& \left\|I_{n-m}\left(\sum_{\boldsymbol{i}_{\boldsymbol{m}} \in \Delta_{n, m}} h_{t, x, n}^{\left(\boldsymbol{i}_{\boldsymbol{m}}\right)}\left(\boldsymbol{s}_{\boldsymbol{m}}, \boldsymbol{y}_{\boldsymbol{m}} ; \bullet\right)\right)\right\|_{2}^{2}=(n-m) !\left\|\sum_{\boldsymbol{i}_{\boldsymbol{m}} \in \Delta_{n, m}} h_{t, x, n}^{\left(\boldsymbol{i}_{\boldsymbol{m}}\right)}\left(\boldsymbol{s}_{\boldsymbol{m}}, \boldsymbol{y}_{\boldsymbol{m}} ; \bullet\right)\right\|_{\mathcal{H}^{\otimes(n-m)}}^{2} \\
& \leq \Gamma_{t}^{n-m}(n-m) !\left\|\sum_{i_{\boldsymbol{m}} \in \Delta_{n, m}} h_{t, x, n}^{\left(i_{\boldsymbol{m}}\right)}\left(\boldsymbol{s}_{\boldsymbol{m}}, \boldsymbol{y}_{\boldsymbol{m}} ; \bullet\right)\right\|_{\mathcal{H}_{0}^{\otimes(n-m)}}^{2}
\end{aligned}
$$




$$
=\Gamma_{t}^{n-m}\left\|I_{n-m}^{\mathfrak{X}}\left(\sum_{\boldsymbol{i}_{\boldsymbol{m}} \in \Delta_{n, m}} h_{t, x, n}^{\left(\boldsymbol{i}_{\boldsymbol{m}}\right)}\left(\boldsymbol{s}_{\boldsymbol{m}}, \boldsymbol{y}_{\boldsymbol{m}} ; \bullet\right)\right)\right\|_{2}^{2} .
$$

Therefore, we get

$$
\left\|D_{\boldsymbol{s}_{\boldsymbol{m}}, y_{\boldsymbol{m}}}^{m} u(t, x)\right\|_{p} \leq \sum_{n \geq m}\left[(p-1) \Gamma_{t}\right]^{\frac{n-m}{2}}\left\|\sum_{\boldsymbol{i}_{\boldsymbol{m}} \in \Delta_{n, m}} I_{n-m}^{\mathfrak{X}}\left(f_{t, x, n}^{\left(\boldsymbol{i}_{\boldsymbol{m}}\right)}\left(\boldsymbol{s}_{\boldsymbol{m}}, \boldsymbol{y}_{\boldsymbol{m}} ; \bullet\right)\right)\right\|_{2}
$$

This leads to

$$
\left\|D_{\boldsymbol{s}_{\boldsymbol{m}}, y_{\boldsymbol{m}}}^{m} u(t, x)\right\|_{p} \leq \sum_{n \geq m}\left[(p-1) \Gamma_{t}\right]^{\frac{n-m}{2}} \sqrt{\mathcal{Q}_{m, n}}
$$

with

$$
\begin{aligned}
\mathcal{Q}_{m, n}: & =\mathbb{E}\left[\left(\sum_{\boldsymbol{i}_{\boldsymbol{m}} \in \Delta_{n, m}} I_{n-m}^{\mathfrak{X}}\left(f_{t, x, n}^{\left(\boldsymbol{i}_{\boldsymbol{m}}\right)}\left(\boldsymbol{s}_{\boldsymbol{m}}, \boldsymbol{y}_{\boldsymbol{m}} ; \bullet\right)\right)\right)^{2}\right] \\
& \leq\left(\begin{array}{c}
n \\
m
\end{array}\right) \sum_{\boldsymbol{i}_{\boldsymbol{m}} \in \Delta_{n, m}} \mathbb{E}\left(I_{n-m}^{\mathfrak{X}}\left(f_{t, x, n}^{\left(\boldsymbol{i}_{\boldsymbol{m}}\right)}\left(\boldsymbol{s}_{\boldsymbol{m}}, \boldsymbol{y}_{\boldsymbol{m}} ; \bullet\right)\right)^{2}\right) .
\end{aligned}
$$

The product formula (2.14) and the decomposition (3.8) yield, with $\left(i_{0}, s_{0}, y_{0}\right)=$ $(0, t, x)$

$$
\begin{aligned}
& \mathcal{Q}_{m, n} \leq\left(\begin{array}{c}
n \\
m
\end{array}\right) \\
& \sum_{i_{m} \in \Delta_{n, m}} \mathbb{E}\left(I_{n-i_{m}}^{\mathfrak{X}}\left(f_{s_{m}, y_{m}, n-i_{m}}\right)^{2} \prod_{j=1}^{m} I_{i_{j}-i_{j-1}-1}^{\mathfrak{X}}\left(f_{s_{j-1}, y_{j-1}, i_{j}-i_{j-1}}^{\left(i_{j}-i_{j-1}\right)}\left(s_{j}, y_{j} ; \bullet\right)\right)^{2}\right) \\
& =\left(\begin{array}{c}
n \\
m
\end{array}\right) \sum_{i_{\boldsymbol{m}} \in \Delta_{n, m}}\left\|I_{n-i_{m}}^{\mathfrak{X}}\left(f_{s_{m}, y_{m}, n-i_{m}}\right)\right\|_{2}^{2} \\
& \quad \times \prod_{j=1}^{m}\left\|I_{i_{j}-i_{j-1}-1}^{\mathfrak{X}}\left(f_{s_{j-1}, y_{j-1}, i_{j}-i_{j-1}}^{\left(i_{j}-i_{j-1}\right)}\left(s_{j}, y_{j} ; \bullet\right)\right)\right\|_{2}^{2}
\end{aligned}
$$

where the last equality is obtained by using the independence among the random variables inside the expectation. It remains to estimate two typical terms:

$$
\left\|I_{j}^{\mathfrak{X}}\left(f_{r, z, j}\right)\right\|_{2}^{2} \text { and }\left\|I_{j-1}^{\mathfrak{X}}\left(f_{t, x, j}^{(j)}(r, z ; \bullet)\right)\right\|_{2}^{2} \text { for } 1 \leq j \leq n \text { and } t>r .
$$


The first term in (3.13) can be estimated as follows. Using Fourier transform in space (see (2.29)), we have, with $t_{0}=r$,

$$
\begin{aligned}
\left\|I_{j}^{\mathfrak{X}}\left(f_{r, z, j}\right)\right\|_{2}^{2} & =j !\left\|\widetilde{f}_{r, z, j}\right\|_{\mathcal{H}_{0}^{\otimes j}}^{2}=\int_{[0, r]^{j}}\left\|f_{r, z, j}\left(\boldsymbol{t}_{\boldsymbol{j}}, \bullet\right)\right\|_{0}^{2} d \boldsymbol{t}_{\boldsymbol{j}} \\
& =\int_{r>t_{1}>\cdots>t_{j}>0} \int_{\mathbb{R}^{d j}}\left|\mathcal{F} f_{r, z, j}\left(\boldsymbol{t}_{\boldsymbol{j}}, \boldsymbol{\xi}_{\boldsymbol{j}}\right)\right|^{2} \mu\left(d \boldsymbol{\xi}_{\boldsymbol{j}}\right) d \boldsymbol{t}_{\boldsymbol{j}} \\
& =\int_{r>t_{1}>\cdots>t_{j}>0}\left(\int_{\mathbb{R}^{d j}} \prod_{k=0}^{j-1}\left|\mathcal{F} G_{t_{k}-t_{k+1}}\left(\xi_{k+1}+\cdots+\xi_{j}\right)\right|^{2} \mu\left(d \xi_{k}\right)\right) d \boldsymbol{t}_{\boldsymbol{j}} .
\end{aligned}
$$

By Lemma 2.6,

$$
\left\|I_{j}^{\mathfrak{X}}\left(f_{r, z, j}\right)\right\|_{2}^{2} \leq \frac{C^{j}}{j !},
$$

where $C=2\left(t^{2}+1\right) \int_{\mathbb{R}^{d}}\left(1+|\xi|^{2}\right)^{-1} \mu(d \xi)$.

Remark 3.1 By the arguments that lead to (3.9), we can also get, for any $p \in[2, \infty)$,

$$
\|u(t, x)\|_{p} \leq 1+\sum_{n \geq 1}\left\|I_{n}\left(f_{t, x, n}\right)\right\|_{p} \leq 1+\sum_{n \geq 1}\left[(p-1) \Gamma_{t}\right]^{n / 2}\left\|I_{n}^{\mathfrak{X}}\left(f_{t, x, n}\right)\right\|_{2}
$$

and then the estimate (3.15) implies $u(t, x) \in L^{p}(\Omega)$. Moreover,

$$
\sup _{(s, y) \in[0, t] \times \mathbb{R}^{d}}\|u(s, y)\|_{p}<+\infty \text { for any } t \in \mathbb{R}_{+} .
$$

This is done under the Dalang's condition (1.2) only and the case $p=2$ provides another proof of [3, Theorem 4.4] when $d=1,2$.

In what follows, we estimate the second term in (3.13) separately for the cases $d=1$ and $d=2$. As usual, we will use $C$ to denote an immaterial constant that may vary from line to line.

3.2.1 Estimation of $\left\|l_{j-1}^{\mathfrak{X}}\left(f_{t, x, j}^{(j)}(r, z ; \bullet)\right)\right\|_{2}^{2}$ when $d=1$

When $d=1, G_{t}(x)=\frac{1}{2} \mathbf{1}_{\{|x|<t\}}$. For $j=1, I_{j-1}^{\mathfrak{X}}\left(f_{t, x, j}^{(j)}(r, z ; \bullet)\right)=G_{t-r}(x-z)$ with the convention (1.6). For $j \geq 2$, it follows from the (modified) isometry property (2.8) that

$$
\left\|I_{j-1}^{\mathfrak{X}}\left(f_{t, x, j}^{(j)}(r, z ; \bullet)\right)\right\|_{2}^{2}=(j-1) !\left\|h_{t, x, j}^{(j)}(r, z ; \bullet)\right\|_{\mathcal{H}_{0}^{\otimes(j-1)}}^{2}
$$




$$
=\int_{[r, t]^{j-1}}\left\|f_{t, x, j}^{(j)}\left(r, z ; \boldsymbol{t}_{\boldsymbol{j}-\mathbf{1}}, \bullet\right)\right\|_{0}^{2} d \boldsymbol{t}_{\boldsymbol{j}-\mathbf{1}},
$$

where we recall that $h_{t, x, j}^{(j)}(r, z ; \bullet)$ is the symmetrization of $f_{t, x, j}^{(j)}(r, z ; \bullet)$; see (3.5). Then, taking advantage of the simple form of $G_{t}(x)$ for $d=1$, we get

$$
0 \leq f_{t, x, j}^{(j)}\left(r, z ; \boldsymbol{t}_{\boldsymbol{j}-\mathbf{1}}, \bullet\right) \leq \frac{1}{2} \mathbf{1}_{\{|x-z|<t-r\}} f_{t, x, j-1}\left(\boldsymbol{t}_{\boldsymbol{j}-\mathbf{1}}, \bullet\right),
$$

from which we further get

$$
\begin{aligned}
\left\|I_{j-1}^{\mathfrak{X}}\left(f_{t, x, j}^{(j)}(r, z ; \bullet)\right)\right\|_{2}^{2} & \leq G_{t-r}^{2}(x-z) \int_{[r, t]^{j-1}}\left\|f_{t, x, j-1}\left(\boldsymbol{t}_{\boldsymbol{j}-\mathbf{1}}, \bullet\right)\right\|_{0}^{2} d \boldsymbol{t}_{\boldsymbol{j}-\mathbf{1}} \\
& \leq \frac{C^{j-1}}{(j-1) !} G_{t-r}^{2}(x-z)
\end{aligned}
$$

where the last inequality follows from (3.15) and (3.14).

\subsubsection{Estimation of $\left\|l_{j-1}^{\mathfrak{X}}\left(f_{t, x, j}^{(j)}(r, z ; \bullet)\right)\right\|_{2}^{2}$ when $d=2$}

Let $q$ be defined as in (2.20) and (2.23) and we fix such a $q$ throughout this subsection. For $j=1, I_{j-1}^{\mathfrak{X}}\left(f_{t, x, j}^{(j)}(r, z ; \bullet)\right)=G_{t-r}(x-z)$ with the convention (1.6). For $j \geq 2$, we begin with

$$
\begin{aligned}
\left\|I_{j-1}^{\mathfrak{X}}\left(f_{t, x, j}^{(j)}(r, z ; \bullet)\right)\right\|_{2}^{2} & =\int_{[r, t]}\left\|f_{t, x, j}^{(j)}\left(r, z ; \boldsymbol{t}_{\boldsymbol{j}-\mathbf{1}}, \bullet\right)\right\|_{0}^{2} d \boldsymbol{t}_{\boldsymbol{j}-\mathbf{1}}, \\
& \leq C^{j-1} \int_{t>t_{1}>\cdots>t_{j-1}>r}\left\|f_{t, x, j}^{(j)}\left(r, z ; \boldsymbol{t}_{\boldsymbol{j}-\mathbf{1}}, \bullet\right)\right\|_{L^{2 q(}\left(\mathbb{R}^{2 j-2}\right)}^{2} d \boldsymbol{t}_{\boldsymbol{j}-\mathbf{1}} \\
& =C^{j-1} \mathcal{T}_{j},
\end{aligned}
$$

where we applied Lemma 2.3 for the inequality above ${ }^{10}$ and we denote

$$
\mathcal{T}_{j}:=\int_{t>t_{1}>\cdots>t_{j-1}>r} d \boldsymbol{t}_{\boldsymbol{j}-\mathbf{1}}\left(\int_{\mathbb{R}^{2(j-1)}} G_{t-t_{1}}^{2 q}\left(x-x_{1}\right) \cdots G_{t_{j-1}-r}^{2 q}\left(x_{j-1}-z\right) d \boldsymbol{x}_{\boldsymbol{j}-\mathbf{1}}\right)^{1 / q} .
$$

Note that we can choose $C$ to depend only on $(t, \gamma, q)$ and be increasing in $t$.

Case $j=2$. In this case, we deduce from Lemma 2.4 and (2.27) that

$$
\mathcal{T}_{2}=\int_{r}^{t} d t_{1}\left(G_{t-t_{1}}^{2 q} * G_{t_{1}-r}^{2 q}\right)^{1 / q}(x-z) \leq C G_{t-r}^{2-\frac{1}{q}}(x-z) \leq C G_{t-r}^{2}(x-z) .
$$

10 The function $\boldsymbol{x}_{\boldsymbol{j}-1} \rightarrow f_{t, x, j}^{(j)}\left(\boldsymbol{t}_{\boldsymbol{j}-\mathbf{1}}, \boldsymbol{x}_{\boldsymbol{j}-1}\right)=G_{t-t_{1}}\left(x-x_{1}\right) G_{t_{1}-t_{2}}\left(x_{1}-x_{2}\right) \ldots G_{t_{j-1}-r}\left(x_{j-1}-z\right)$ has support contained in $\left\{\boldsymbol{x}_{\boldsymbol{j}-1} \in \mathbb{R}^{2(j-1)} ;\left|x_{i}-x\right|<t-t_{i}\right.$, for all $\left.i=1, \ldots, j-1\right\}$. 
Case $j \geq 3$. In this case, we use Minkowski inequality with respect to the norm in $L^{1 / q}\left(\left[t_{2}, t\right], d t_{1}\right)$ in order to get

$$
\begin{aligned}
\mathcal{T}_{j} \leq & \int_{t>t_{2}>\cdots>t_{j-1}>r}\left(\int_{\mathbb{R}^{2(j-2)}}\left[\int_{t_{2}}^{t}\left(G_{t-t_{1}}^{2 q} * G_{t_{1}-t_{2}}^{2 q}\right)^{1 / q}\left(x-x_{2}\right) d t_{1}\right]^{q}\right. \\
& \left.\times G_{t_{2}-t_{3}}^{2 q}\left(x_{2}-x_{3}\right) \cdots G_{t_{j-1}-r}^{2 q}\left(x_{j-1}-z\right) d x_{2} \cdots d x_{j-1}\right)^{1 / q} d t_{2} \cdots d t_{j-1} .
\end{aligned}
$$

Applying Lemma 2.4 yields

$$
\begin{aligned}
\mathcal{T}_{j} \leq & A_{q} \int_{t>t_{2}>\cdots>t_{j-1}>r}\left(t-t_{2}\right)^{\frac{1}{q}-1}\left(\int_{\mathbb{R}^{2(j-2)}} G_{t-t_{2}}^{2 q-1}\left(x-x_{2}\right)\right. \\
& \left.\times G_{t_{2}-t_{3}}^{2 q}\left(x_{2}-x_{3}\right) \cdots G_{t_{j-1}-r}^{2 q}\left(x_{j-1}-z\right) d x_{2} \cdots d x_{j-1}\right)^{1 / q} d t_{2} \cdots d t_{j-1} .
\end{aligned}
$$

If $j=3$, we have

$$
\mathcal{T}_{3} \leq A_{q} \int_{r}^{t}\left(t-t_{2}\right)^{\frac{1}{q}-1}\left(\int_{\mathbb{R}^{2}} G_{t-t_{2}}^{2 q-1}\left(x-x_{2}\right) G_{t_{2}-r}^{2 q}\left(x_{2}-z\right) d x_{2}\right)^{1 / q} d t_{2}
$$

Owing to (2.27), we can bound $G_{t-t_{2}}^{2 q-1}\left(x-x_{2}\right)$ by $(2 \pi)\left(t-t_{2}\right) G_{t-t_{2}}^{2 q}\left(x-x_{2}\right)$, and then we apply again Lemma 2.4 and (2.27) to conclude that

$$
\mathcal{T}_{3} \leq A_{q}^{2}(2 \pi)^{\frac{1}{q}}(t-r)^{\frac{3}{q}-2} G_{t-r}^{2-\frac{1}{q}}(x-z) \leq C G_{t-r}^{2}(x-z) .
$$

For $j \geq 4$, we continue with the estimate (3.20). We can first apply Minkowski inequality with respect to the norm $L^{1 / q}\left(\left[t_{4}, t_{2}\right], d t_{3}\right)$ and then apply Lemma 2.4 to obtain

$$
\begin{aligned}
\mathcal{T}_{j} \leq & A_{q}^{2} \int_{t>t_{2}>t_{4}>\cdots>t_{j-1}>r} d t_{2} d t_{4} \cdots d t_{j-1}\left(t-t_{2}\right)^{\frac{1}{q}-1}\left(t_{2}-t_{4}\right)^{\frac{1}{q}-1} \\
& \left(\int_{\mathbb{R}^{2(j-3)}} G_{t-t_{2}}^{2 q-1}\left(x-x_{2}\right)\right. \\
& \left.\times G_{t_{2}-t_{4}}^{2 q-1}\left(x_{2}-x_{4}\right) G_{t_{4}-t_{5}}^{2 q}\left(x_{4}-x_{5}\right) \cdots G_{t_{j-1}-r}^{2 q}\left(x_{j-1}-z\right) d x_{2} d x_{4} \cdots d x_{j-1}\right)^{1 / q} .
\end{aligned}
$$

Note that

$$
G_{t-t_{2}}^{2 q-1}\left(x-x_{2}\right) G_{t_{2}-t_{4}}^{2 q-1}\left(x_{2}-x_{4}\right) \leq \mathbf{1}_{\left\{\left|x-x_{4}\right| \leq t-t_{4}\right\}} G_{t-t_{2}}^{2 q-1}\left(x-x_{2}\right) G_{t_{2}-t_{4}}^{2 q-1}\left(x_{2}-x_{4}\right) .
$$


Then, by Cauchy-Schwarz inequality and (2.26), we can infer that

$$
\begin{aligned}
\int_{\mathbb{R}^{2}} G_{t-t_{2}}^{2 q-1}\left(x-x_{2}\right) G_{t_{2}-t_{4}}^{2 q-1}\left(x_{2}-x_{4}\right) d x_{2} & \leq \mathbf{1}_{\left\{\left|x-x_{4}\right| \leq t-t_{4}\right\}}\left\|G_{t-t_{2}}^{2 q-1}\right\|_{L^{2}\left(\mathbb{R}^{2}\right)}\left\|G_{t_{2}-t_{4}}^{2 q-1}\right\|_{L^{2}\left(\mathbb{R}^{2}\right)} \\
& =c_{1}\left(t-t_{2}\right)^{2-2 q}\left(t_{2}-t_{4}\right)^{2-2 q} \mathbf{1}_{\left\{\left|x-x_{4}\right| \leq t-t_{4}\right\}},
\end{aligned}
$$

where $c_{1}=\frac{(2 \pi)^{3-4 q}}{4-4 q}$. Thus, substituting this estimate into (3.22), we end up with

$$
\begin{aligned}
\mathcal{T}_{j} & \leq A_{q}^{2} c_{1}^{1 / q} \int_{t>t_{2}>t_{4}>\cdots>t_{j-1}>r} d t_{2} d t_{4} \cdots d t_{j-1}\left(t-t_{2}\right)^{\frac{3}{q}-3}\left(t_{2}-t_{4}\right)^{\frac{3}{q}-3} \\
& \times\left(\int_{\mathbb{R}^{2(j-4)}} \mathbf{1}_{\left\{\left|x-x_{4}\right| \leq t-t_{4}\right\}} G_{t_{4}-t_{5}}^{2 q}\left(x_{4}-x_{5}\right) \cdots G_{t_{j-1}-r}^{2 q}\left(x_{j-1}-z\right) d x_{4} \cdots d x_{j-1}\right)^{1 / q} .
\end{aligned}
$$

Focusing on the indicators, the right-hand side of this estimate can be bounded by

$$
\begin{aligned}
& A_{q}^{2} c_{1}^{1 / q} \mathbf{1}_{\{|x-z| \leq t-r\}} \int_{t>t_{2}>t_{4}>\cdots>t_{j-1}>r} d t_{2} d t_{4} \cdots d t_{j-1}\left(t-t_{2}\right)^{\frac{3}{q}-3}\left(t_{2}-t_{4}\right)^{\frac{3}{q}-3} \\
& \quad \times\left(\int_{\mathbb{R}^{2(j-4)}} G_{t_{4}-t_{5}}^{2 q}\left(x_{4}-x_{5}\right) \cdots G_{t_{j-1}-r}^{2 q}\left(x_{j-1}-z\right) d x_{4} \cdots d x_{j-1}\right)^{1 / q} .
\end{aligned}
$$

For $j=4$, using (2.28), we have

$$
\mathcal{T}_{4} \leq A_{q}^{2} c_{1}^{1 / q}(t-r)^{\frac{6}{q}-6} \mathbf{1}_{\{|x-z| \leq t-r\}} \leq C G_{t-r}^{2}(x-z)
$$

Now for $j \geq 5$, we just integrate in each of the variables $x_{4}, \ldots, x_{j-1}$ (with this order) so that, thanks to (2.26), we end up with

$$
\begin{aligned}
& \mathcal{T}_{j} \leq A_{q}^{2} c_{1}^{1 / q} c_{2}^{j-4} \mathbf{1}_{\{|x-z| \leq t-r\}} \int_{t>t_{2}>t_{4}>\cdots>t_{j-1}>r} d t_{2} d t_{4} \cdots d t_{j-1} \\
& \times\left(t-t_{2}\right)^{\frac{3}{q}-3}\left(t_{2}-t_{4}\right)^{\frac{3}{q}-3}\left(t_{4}-t_{5}\right)^{\frac{2}{q}-2} \cdots\left(t_{j-1}-r\right)^{\frac{2}{q}-2} \text { with } c_{2} \\
& =\left(\frac{(2 \pi)^{1-2 q}}{2-2 q}\right)^{2} \leq A_{q}^{2} c_{1}^{1 / q} c_{2}^{j-4} \frac{(t-r)^{j-3}}{(j-3) !}(t-r+1)^{j\left(\frac{2}{q}-2\right)} \mathbf{1}_{\{|x-z| \leq t-r\}},
\end{aligned}
$$

where we used the rough estimate $a^{v} \leq(b+1)^{v}$ for $0<a \leq b$ and $v>0$. Thus, using (2.28) we obtain:

$$
\mathcal{T}_{j} \leq \frac{C^{j-3}}{(j-3) !} G_{t-r}^{2}(x-z) \text { for any } j \geq 5
$$


Hence, combining the estimates (3.19), (3.21), (3.23) and (3.24) and taking into account that $I_{0}^{\mathfrak{X}}\left(f_{t, x, 1}^{(1)}(r, z ; \bullet)\right)=G_{r-s}(z-y)$, we can write

$$
\left\|I_{j-1}^{\mathfrak{X}}\left(f_{t, x, j}^{(j)}(r, z ; \bullet)\right)\right\|_{2}^{2} \leq\left\{\begin{array}{ll}
C G_{t-r}^{2}(x-z) & \text { for } j=1,2,3,4 \\
\frac{C^{j}}{(j-3) !} G_{t-r}^{2}(x-z) & \text { for } j \geq 5
\end{array},\right.
$$

where the constant $C>1$ depends on $(t, \gamma, q)$ and is increasing in $t$. For $1 \leq j \leq n$, we obtain the following bound

$$
\left\|I_{j-1}^{\mathfrak{X}}\left(f_{t, x, j}^{(j)}(r, z ; \bullet)\right)\right\|_{2}^{2} \leq \frac{C^{j}}{j !} n^{3} G_{t-r}^{2}(x-z) .
$$

\subsection{Step 3: Proof of (1.11)}

Let us first consider the lower bound in (1.11) for $d \in\{1,2\}$. For $p \in[2, \infty)$, we deduce from the modified isometry (2.8) that

$$
\left\|D_{\boldsymbol{s}_{\boldsymbol{m}}, y_{\boldsymbol{m}}}^{m} u(t, x)\right\|_{p} \geq\left\|D_{\boldsymbol{s}_{\boldsymbol{m}}, y_{\boldsymbol{m}}}^{m} u(t, x)\right\|_{2} \geq m ! \widetilde{f}_{t, x, m}\left(\boldsymbol{s}_{\boldsymbol{m}}, \boldsymbol{y}_{\boldsymbol{m}}\right)
$$

Now let us establish the upper bound in (1.11). By symmetry, we can assume $t>s_{1}>$ $\cdots>s_{m}>0$. First we consider the case where $d=2$. Recall the definition of $\mathcal{Q}_{m, n}$ from (3.11), and then plugging the estimates (3.15) and (3.25) into (3.12) yields, with $\left(i_{0}, s_{0}, y_{0}\right)=(0, t, x)$,

$$
\begin{aligned}
\mathcal{Q}_{m, n} & \leq\left(\begin{array}{c}
n \\
m
\end{array}\right) \sum_{i_{\boldsymbol{m}} \in \Delta_{n, m}} \frac{C^{n-i_{m}}}{\left(n-i_{m}\right) !} \times \prod_{j=1}^{m} \frac{n^{3} C^{i_{j}-i_{j-1}}}{\left(i_{j}-i_{j-1}\right) !} G_{s_{j-1}-s_{j}}^{2}\left(y_{j-1}-y_{j}\right) \\
& \leq(2 C)^{n} n^{3 m}\left(\sum_{i_{\boldsymbol{m}} \in \Delta_{n, m}} \frac{1}{i_{1} !\left(i_{2}-i_{1}\right) ! \cdots\left(i_{m}-i_{m-1}\right) !\left(n-i_{m}\right) !}\right) f_{t, x, m}^{2}\left(\boldsymbol{s}_{\boldsymbol{m}}, \boldsymbol{y}_{\boldsymbol{m}}\right),
\end{aligned}
$$

where we used the rough bound $\left(\begin{array}{l}n \\ m\end{array}\right) \leq 2^{n}$. The sum in the above display is equal to

$$
\frac{1}{n !} \sum_{\substack{a_{1}+\ldots+a_{m+1}=n \\
a_{i} \in \mathbb{N}, \forall i}}\left(\begin{array}{c}
n \\
a_{1}, \ldots, a_{m+1}
\end{array}\right)=\frac{(m+1)^{n}}{n !}
$$

by multinomial formula. That is, we can get

$$
\mathcal{Q}_{m, n} \leq \frac{[C(m+1)]^{n} n^{3 m}}{n !} f_{t, x, m}^{2}\left(\boldsymbol{s}_{\boldsymbol{m}}, \boldsymbol{y}_{\boldsymbol{m}}\right)
$$

which, together with the estimate (3.10), implies the upper bound in (1.11), when $d=2$. 
The case $d=1$ can be done in the same way by noticing that the bound in (3.17) can be replaced by $n \frac{C^{j}}{j !} G_{t-r}^{2}(x-z)$ for $1 \leq j \leq n$. Then, like the estimate for $d=2$, we can get, for $t>s_{1}>\cdots>s_{m}>0$,

$$
\mathcal{Q}_{m, n} \leq \frac{[C(m+1)]^{n} n^{m}}{n !} f_{t, x, m}^{2}\left(\boldsymbol{s}_{\boldsymbol{m}}, \boldsymbol{y}_{\boldsymbol{m}}\right),
$$

which together with the estimate (3.10) implies the upper bound in (1.11), when $d=1$. This completes the proof of the estimate (1.11).

Notice that the upper bound also shows the convergence in $L^{p}$ for any $p \in[2, \infty)$ of the series (3.6), for any fixed $\boldsymbol{s}_{\boldsymbol{m}} \in[0, t]^{m}$ and $\boldsymbol{y}_{\boldsymbol{m}} \in \mathbb{R}^{d m}$.

\subsection{Step 4: Existence of a measurable version}

We claim that there is a random field $Y$ such that $Y\left(\boldsymbol{s}_{\boldsymbol{m}}, \boldsymbol{y}_{\boldsymbol{m}}\right)=D_{\boldsymbol{s}_{\boldsymbol{m}}, y_{\boldsymbol{m}}}^{m} u(t, x)$ almost surely for almost all $\left(\boldsymbol{s}_{\boldsymbol{m}}, \boldsymbol{y}_{\boldsymbol{m}}\right) \in[0, t]^{m} \times \mathbb{R}^{m d}$ and the mapping

$$
\left(\omega, \boldsymbol{s}_{\boldsymbol{m}}, \boldsymbol{y}_{\boldsymbol{m}}\right) \in \Omega \times[0, t]^{m} \times \mathbb{R}^{m d} \longmapsto Y\left(\omega, \boldsymbol{s}_{\boldsymbol{m}}, \boldsymbol{y}_{\boldsymbol{m}}\right) \in \mathbb{R}
$$

is jointly measurable. This fact is rather standard and we will sketch the proof only in the case $d=2$. From the explicit form of the kernels $f_{t, x, n}$ given in (1.8), it follows that the mapping

$$
\left(s_{\boldsymbol{m}}, \boldsymbol{y}_{\boldsymbol{m}}\right) \rightarrow{\widetilde{f_{t, x, n}}}\left(\boldsymbol{s}_{\boldsymbol{m}}, \boldsymbol{y}_{\boldsymbol{m}} ; \bullet\right)
$$

is measurable from $[0, t]^{m} \times \mathbb{R}^{2 m}$ to $L^{2}\left([0, t]^{n-m} ; L^{2 q}\left(\mathbb{R}^{2(n-m)}\right)\right)$. Because

$L^{2}\left([0, t]^{n-m} ; L^{2 q}\left(\mathbb{R}^{2(n-m)}\right)\right)$ is continuously embedded into $\mathcal{H}^{\otimes(n-m)}$ (see (2.13) and $(2.25))$,

we deduce that the map (3.26) is measurable from $[0, t]^{m} \times \mathbb{R}^{2 m}$ into $\mathcal{H}^{\otimes(n-m)}$. This implies that the mapping

$$
\left(\boldsymbol{s}_{\boldsymbol{m}}, \boldsymbol{y}_{\boldsymbol{m}}\right) \rightarrow I_{n-m}\left(\widetilde{f_{t, x, n}}\left(\boldsymbol{s}_{\boldsymbol{m}}, \boldsymbol{y}_{\boldsymbol{m}} ; \bullet\right)\right)
$$

is measurable from $[0, t]^{m} \times \mathbb{R}^{2 m}$ to $L^{2}(\Omega)$. The upper bound in (1.11) implies that the mapping (3.27) belongs to the space

$$
L^{2 q}\left([0, t]^{m} \times \mathbb{R}^{2 m} ; L^{2}(\Omega)\right) \subset L^{2 q}\left([0, t]^{m} \times \mathbb{R}^{2 m} \times \Omega\right) .
$$

From this, it follows that we can find a measurable modification of the process

$$
\left\{I_{n-m}\left(\widetilde{f}_{t, x, n}\left(\boldsymbol{s}_{\boldsymbol{m}}, \boldsymbol{y}_{\boldsymbol{m}} ; \bullet\right)\right)(\omega):\left(\omega, \boldsymbol{s}_{\boldsymbol{m}}, \boldsymbol{y}_{\boldsymbol{m}}\right) \in \Omega \times[0, t]^{m} \times \mathbb{R}^{2 m}\right\} .
$$

Finally, by standard arguments we deduce the existence of a measurable modification of the series (3.6). 


\subsection{Step 5: Proof of $u(t, x) \in \mathbb{D}^{\infty}$}

We have already seen in Remark 3.1 that $u(t, x) \in L^{p}(\Omega)$ for any $p \in[2, \infty)$. Then, it remains to show that the function $D_{s_{m}, y_{m}}^{m} u(t, x)$ defined as the limit of the series (3.6) coincides with the $m$ th Malliavin derivative of $u(t, x)$. To do this, it suffices to show that $\mathbb{E}\left[\left\|D^{m} u(t, x)\right\|_{\mathcal{H}^{\otimes m}}^{p}\right]<\infty$ for any $m \geq 1$. By Fubini' theorem and using the upper bound (1.11), we write

$$
\begin{aligned}
(\mathbb{E} & {\left.\left[\left\|D^{m} u(t, x)\right\|_{\mathcal{H}^{\otimes m}}^{p}\right]\right)^{2 / p} } \\
= & \| \int_{[0, t]^{2 m} \times \mathbb{R}^{2 m d}} d \boldsymbol{s}_{\boldsymbol{m}} d \boldsymbol{s}_{\boldsymbol{m}}^{\prime} d \boldsymbol{y}_{\boldsymbol{m}} d \boldsymbol{y}_{\boldsymbol{m}}^{\prime}\left(D_{\boldsymbol{s}_{\boldsymbol{m}}, y_{\boldsymbol{m}}}^{m} u(t, x)\right)\left(D_{\boldsymbol{s}_{\boldsymbol{m}}^{\prime}, \boldsymbol{y}_{\boldsymbol{m}}^{\prime}}^{m} u(t, x)\right) \\
& \prod_{j=1}^{m} \gamma_{0}\left(s_{j}-s_{j}^{\prime}\right) \gamma\left(y_{j}-y_{j}^{\prime}\right) \|_{p / 2} \\
\leq & \int_{[0, t]^{2 m} \times \mathbb{R}^{2 m d}} d \boldsymbol{s}_{\boldsymbol{m}} d \boldsymbol{s}_{\boldsymbol{m}}^{\prime} d \boldsymbol{y}_{\boldsymbol{m}} d \boldsymbol{y}_{\boldsymbol{m}}^{\prime}\left\|D_{\boldsymbol{s}_{\boldsymbol{m}}, \boldsymbol{y}_{\boldsymbol{m}}}^{m} u(t, x)\right\|_{p}\left\|D_{\boldsymbol{s}_{\boldsymbol{m}}^{\prime}, \boldsymbol{y}_{\boldsymbol{m}}^{\prime}}^{m} u(t, x)\right\|_{p} \\
& \prod_{j=1}^{m} \gamma_{0}\left(s_{j}-s_{j}^{\prime}\right) \gamma\left(y_{j}-y_{j}^{\prime}\right) \\
& \lesssim\left\|\widetilde{f}_{t, x, m}\right\|_{\mathcal{H}^{\otimes m}}^{2}<\infty .
\end{aligned}
$$

This shows $u(t, x) \in \mathbb{D}^{\infty}$ and completes the proof of Theorem 1.3.

Remark 3.2 When $d=2, p=2, m=1$ and for the cases (a), (b) in Hypothesis (H1), the upper bound in (1.11) can be proved in a much simpler way for almost all $(r, z) \in[0, t] \times \mathbb{R}^{2}$. Let $v_{\lambda}$ be the solution to the stochastic wave equation

$$
\left\{\begin{array}{l}
\frac{\partial^{2} v_{\lambda}}{\partial t^{2}}=\Delta v_{\lambda}+\lambda v_{\lambda} \dot{\mathfrak{X}} \\
v_{\lambda}(0, \bullet)=1, \quad \frac{\partial v_{\lambda}}{\partial t}(0, \bullet)=0,
\end{array}\right.
$$

where $\lambda>0$ and $\dot{\mathcal{X}}$ is given as before. This solution has the chaos expansion $v_{\lambda}(t, x)=$ $\sum_{n \geq 0} \lambda^{n} I_{n}^{\mathfrak{X}}\left(f_{t, x, n}\right)$ and its Malliavin derivative has the chaos expansion

$$
D_{r, z} v_{\lambda}(t, x)=\sum_{n \geq 1} \lambda^{n} I_{n-1}^{\mathfrak{X}}\left(\sum_{j=1}^{n} h_{t, x, n}^{(j)}(r, z ; \bullet)\right) ;
$$

see (3.1) and (3.2). From this, we infer that for any $(\lambda, t, x) \in(0, \infty)^{2} \times \mathbb{R}^{2}$ and for almost every $(r, z) \in[0, t] \times \mathbb{R}^{2}$,

$$
\left\|D_{r, z} v_{\lambda}(t, x)\right\|_{2}^{2}=\sum_{n \geq 1}(n-1) ! \lambda^{2 n}\left\|\sum_{j=1}^{n} h_{t, x, n}^{(j)}(r, z ; \bullet)\right\|_{\mathcal{H}_{0}^{\otimes(n-1)}}^{2} \leq C_{\lambda, t, \gamma} G_{t-r}^{2}(x-z),
$$


where $C_{\lambda, t, \gamma}>0$ is a constant depending on $(\lambda, t, \gamma)$ and is increasing in $t$. The inequality above is due to Theorem 1.3 of [35] for case (a), respectively Theorem 1.2 of [4] for case (b). Therefore,

$$
\begin{aligned}
\left\|D_{r, z} u(t, x)\right\|_{2}^{2} & =\sum_{n \geq 1}(n-1) !\left\|\sum_{j=1}^{n} h_{t, x, n}^{(j)}(r, z ; \bullet)\right\|_{\mathcal{H}^{\otimes(n-1)}}^{2} \\
& \leq \sum_{n \geq 1}(n-1) ! \Gamma_{t}^{n-1}\left\|\sum_{j=1}^{n} h_{t, x, n}^{(j)}(r, z ; \bullet)\right\|_{\mathcal{H}_{0}^{\otimes(n-1)}}^{2} \text { by (2.13). }
\end{aligned}
$$

Thus, using (3.28) with $\lambda=\sqrt{\Gamma_{t}}$, we get $\left\|D_{r, z} u(t, x)\right\|_{2}^{2} \leq C_{\Gamma_{t}, t, \gamma} G_{t-r}^{2}(x-z)$.

\subsection{Consequences of Theorem 1.3}

We will establish two estimates that will be useful in Sect. 5 .

Corollary 3.3 Let $d=1,2$. Then, for any finite $T>0$,

$$
\sup _{(t, x) \in[0, T] \times \mathbb{R}^{d}} \sup _{r \in[0, t]} \mathbb{E}\left[\left\|\left|D_{r, \bullet} u(t, x)\right|\right\|_{0}^{2}\right]<\infty .
$$

In particular, $D_{r, \bullet} u(t, x)(\omega) \in\left|\mathcal{P}_{0}\right|$ for almost every $(\omega, r) \in \Omega \times[0, t]$, where $\left|\mathcal{P}_{0}\right|$ is defined in (2.2).

Proof We work with a version of $\left\{D_{r, z} u(t, x):(r, z) \in[0, t] \times \mathbb{R}^{2}\right\}$ that is jointly measurable. By Fubini's theorem and Cauchy-Schwarz inequality, we have

$$
\begin{aligned}
\mathbb{E}\left[\left\|\left|D_{r, \bullet} u(t, x)\right|\right\|_{0}^{2}\right] & \leq \mathbb{E} \int_{\mathbb{R}^{2 d}}\left|D_{r, z} u(t, x) \| D_{r, z^{\prime}} u(t, x)\right| \gamma\left(z-z^{\prime}\right) d z d z^{\prime} \\
& \leq \int_{\mathbb{R}^{2 d}}\left\|D_{r, z} u(t, x)\right\|_{2}\left\|D_{r, z^{\prime}} u(t, x)\right\|_{2} \gamma\left(z-z^{\prime}\right) d z d z^{\prime} \\
& \leq C \int_{\mathbb{R}^{2 d}} G_{t-r}(x-z) G_{t-r}\left(x-z^{\prime}\right) \gamma\left(z-z^{\prime}\right) d z d z^{\prime} \text { by Theorem } 1.3 \\
& =C \int_{\mathbb{R}^{d}} \mu(d \xi)\left|\widehat{G}_{t-r}(\xi)\right|^{2} \text { using Fourier transform } \\
& \leq 2 C\left(t^{2} \vee 1\right) \int_{\mathbb{R}^{d}} \frac{\mu(d \xi)}{1+|\xi|^{2}} \text { by }(2.33),
\end{aligned}
$$

where $C$ is a constant depending on $\gamma_{0}, \gamma, t$ and is increasing in $t$. The above (uniform) bound implies (3.29). Hence, $D_{r, \bullet} u(t, x)(\omega) \in\left|\mathcal{P}_{0}\right|$ for almost all $(\omega, r) \in \Omega \times[0, t]$. 
The space $\left|\mathcal{H} \otimes \mathcal{P}_{0}\right|$ appearing in the next corollary is defined as the set of measurable functions $h: \mathbb{R}_{+} \times \mathbb{R}^{2 d} \rightarrow \mathbb{R}$ such that

$$
\begin{aligned}
& \int_{\mathbb{R}_{+}^{2} \times \mathbb{R}^{4 d}}|h(r, w, z)|\left|h\left(r^{\prime}, w^{\prime}, z^{\prime}\right)\right| \\
& \quad \gamma_{0}\left(r-r^{\prime}\right) \gamma\left(w-w^{\prime}\right) \gamma\left(z-z^{\prime}\right) d w d w^{\prime} d z d z^{\prime} d r d r^{\prime}<\infty .
\end{aligned}
$$

Then, $\left|\mathcal{H} \otimes \mathcal{P}_{0}\right| \subset \mathcal{H} \otimes \mathcal{P}_{0}$

Corollary 3.4 Let $d=1,2$. For almost all $(\omega, r) \in \Omega \times[0, t], D D_{r, \bullet} u(t, x)(\omega) \in$ $\left|\mathcal{H} \otimes \mathcal{P}_{0}\right|$ and for any finite $T>0$,

$$
\sup _{(t, x) \in[0, T] \times \mathbb{R}^{d}} \sup _{r \in[0, t]} \mathbb{E}\left(\left\|\left|D D_{r, \bullet} u(t, x)\right|\right\|_{\mathcal{H} \otimes \mathcal{P}_{0}}^{2}\right)<+\infty .
$$

Proof Using Theorem 1.3, Cauchy-Schwarz inequality and the estimate (1.11), we can write

$$
\begin{aligned}
\mathbb{E} & \left(\left\|\left|D D_{r, \bullet} u(t, x)\right|\right\|_{\mathcal{H} \otimes \mathcal{P}_{0}}^{2}\right) \\
= & \mathbb{E}\left(\int_{[0, t]^{2}} \int_{\mathbb{R}^{4 d}}\left|D_{(\theta, w),(r, z)}^{2} u(t, x) \| D_{\left(\theta^{\prime}, w^{\prime}\right),\left(r, z^{\prime}\right)}^{2} u(t, x)\right|\right. \\
& \left.\times \gamma_{0}\left(\theta-\theta^{\prime}\right) \gamma\left(w-w^{\prime}\right) \gamma\left(z-z^{\prime}\right) d w d w^{\prime} d z d z^{\prime} d \theta d \theta^{\prime}\right) \\
\leq & \int_{[0, t]^{2}} \int_{\mathbb{R}^{4 d}}\left\|D_{(\theta, w),(r, z)}^{2} u(t, x)\right\|_{2}\left\|D_{\left(\theta^{\prime}, w^{\prime}\right),\left(r, z^{\prime}\right)}^{2} u(t, x)\right\|_{2} \\
& \times \gamma_{0}\left(\theta-\theta^{\prime}\right) \gamma\left(w-w^{\prime}\right) \gamma\left(z-z^{\prime}\right) d w d w^{\prime} d z d z^{\prime} d \theta d \theta^{\prime} \\
\leq & C \int_{[0, t]^{2}} \int_{\mathbb{R}^{4 d}} \widetilde{f}_{t, x, 2}(r, z, \theta, w) \widetilde{f}_{t, x, 2}\left(r, z^{\prime}, \theta^{\prime}, w^{\prime}\right) \\
& \gamma_{0}\left(\theta-\theta^{\prime}\right) \gamma\left(w-w^{\prime}\right) \gamma\left(z-z^{\prime}\right) d w d w^{\prime} d z d z^{\prime} d \theta d \theta^{\prime} .
\end{aligned}
$$

As a consequence,

$$
\begin{aligned}
& \mathbb{E}\left(\left\|\left|D D_{r, \bullet} u(t, x)\right|\right\|_{\mathcal{H} \otimes \mathcal{P}_{0}}^{2}\right) \\
& \quad \leq C \int_{\mathbb{R}^{2 d}}\left\|\widetilde{f}_{t, x, 2}(r, z ; \bullet)\right\|_{\mathcal{H}}\left\|\widetilde{f}_{t, x, 2}\left(r, z^{\prime} ; \bullet\right)\right\|_{\mathcal{H}} \gamma\left(z-z^{\prime}\right) d z d z^{\prime} .
\end{aligned}
$$

By the arguments used in the proof of Theorem 1.3, it follows that

$$
\left\|\widetilde{f_{t, x, 2}}(r, z ; \bullet)\right\|_{\mathcal{H}} \leq C G_{t-r}(x-z)
$$


Therefore,

$$
\mathbb{E}\left(\left\|\left|D D_{r, \bullet} u(t, x)\right|\right\|_{\mathcal{H} \otimes \mathcal{P}_{0}}^{2}\right) \leq C \int_{\mathbb{R}^{2 d}} \gamma\left(z-z^{\prime}\right) G_{t-r}(x-z) G_{t-r}\left(x-z^{\prime}\right) d z d z^{\prime}
$$

and the same argument as in the proof of Corollary 3.3 ends our proof.

Remark 3.5 Note that for any finite $T>0, \mathbb{E}\left(\left\|\left|D^{2} u(t, x)\right|\right\|_{\mathcal{H}^{\otimes 2}}^{2}\right)<\infty$ for any $(t, x) \in[0, T] \times \mathbb{R}^{d}$.

\section{Gaussian fluctuation: Proof of Theorem 1.4}

Recall that

$$
F_{R}(t)=\int_{B_{R}}[u(t, x)-1] d x
$$

and $\sigma_{R}(t)=\sqrt{\operatorname{Var}\left(F_{R}(t)\right)}$. First, we need to obtain the limiting covariance structure, which is the content of Proposition 4.1. It will give us the growth order of $\sigma_{R}(t)$. Then, in Sect. 4.2, we apply the second-order Gaussian Poincaré inequality to establish the quantitative CLT for $F_{R}(t) / \sigma_{R}(t)$. Finally, we will prove the functional CLT by showing the convergence of the finite-dimensional distributions and the tightness.

\subsection{Limiting covariance}

Proposition 4.1 Let $u$ denote the solution to the hyperbolic Anderson model (1.1) and assume that the non-degeneracy condition (1.17) holds. Then, the following results hold true:

(1) Suppose $d \in\{1,2\}$ and $\gamma\left(\mathbb{R}^{d}\right) \in(0, \infty)$. Then, for any $t, s \in(0, \infty)$,

$$
\lim _{R \rightarrow \infty} R^{-d} \mathbb{E}\left[F_{R}(t) F_{R}(s)\right]=\omega_{d} \sum_{p \geq 1} p ! \int_{\mathbb{R}^{d}}\left\langle\widetilde{f}_{t, x, p}, \widetilde{f}_{s, 0, p}\right\rangle_{\mathcal{H}^{\otimes p}} d x
$$

see also (1.18). In particular, $\sigma_{R}(t) \sim R^{d / 2}$.

(2) Suppose $d \in\{1,2\}$ and $\gamma(x)=|x|^{-\beta}$ for some $\beta \in(0,2 \wedge d)$. Then, for any $t, s \in(0, \infty)$,

$$
\lim _{R \rightarrow \infty} R^{\beta-2 d} \mathbb{E}\left[F_{R}(t) F_{R}(s)\right]=\kappa_{\beta, d} \int_{0}^{t} d r \int_{0}^{s} d r^{\prime} \gamma_{0}\left(r-r^{\prime}\right)(t-r)\left(s-r^{\prime}\right),
$$

where $\kappa_{\beta, d}=\int_{B_{1}^{2}} d x d y|x-y|^{-\beta}$ is introduced in (1.16). In particular, $\sigma_{R}(t) \sim$ $R^{d-\frac{\beta}{2}}$. 
(3) Suppose $d=2$ and $\gamma\left(x_{1}, x_{2}\right)=\gamma_{1}\left(x_{1}\right) \gamma_{2}\left(x_{2}\right)$ satisfies one of the following conditions:

$$
\left\{\begin{array}{l}
\left(c_{1}\right) \quad \gamma_{i}\left(x_{i}\right)=\left|x_{i}\right|^{-\beta_{i}} \text { for some } \beta_{i} \in(0,1), i=1,2 ; \\
\left(c_{2}\right) \quad \gamma_{1} \in L^{1}(\mathbb{R}) \text { and } \gamma_{2}(x)=|x|^{-\beta} \text { for some } \beta \in(0,1)
\end{array} .\right.
$$

For any $s, t \in(0, \infty)$, the following results hold true:

$\left(r_{1}\right)$ In $\left(c_{1}\right)$, we have

$$
\begin{aligned}
& \lim _{R \rightarrow \infty} R^{\beta_{1}-\beta_{2}-4} \mathbb{E}\left[F_{R}(t) F_{R}(s)\right] \\
& =K_{\beta_{1}, \beta_{2}} \int_{0}^{t} d r \int_{0}^{s} d r^{\prime} \gamma_{0}\left(r-r^{\prime}\right)(t-r)\left(s-r^{\prime}\right),
\end{aligned}
$$

where $K_{\beta_{1}, \beta_{2}}$ is defined in (1.22).

$\left(r_{2}\right)$ In $\left(c_{2}\right)$, we have

$$
\lim _{R \rightarrow \infty} R^{\beta-3} \mathbb{E}\left[F_{R}(t) F_{R}(s)\right]=\gamma_{1}(\mathbb{R}) \mathcal{L}_{\beta} \int_{0}^{t} d r \int_{0}^{s} d r^{\prime} \gamma_{0}\left(r-r^{\prime}\right)(t-r)\left(s-r^{\prime}\right),
$$

where $\mathcal{L}_{\beta}$ is defined in (1.24).

\subsubsection{Proof of part (1) in Proposition 4.1}

Preparation. In the following, we will denote by $\varphi$ the density of $\mu$. For $0<s \leq t<$ $\infty$ and $x, y \in \mathbb{R}^{d}$, we have

$$
\begin{aligned}
\mathbb{E}[u(t, x) u(s, y)]-1 & =\sum_{p \geq 1} p !\left\langle\widetilde{f}_{t, x, p}, \tilde{f}_{s, y, p}\right\rangle_{\mathcal{H} \otimes p} \\
& =: \sum_{p \geq 1} \frac{1}{p !} \Phi_{p}(t, s ; x-y),
\end{aligned}
$$

where $\widetilde{f}_{t, x, p} \in \mathcal{H}^{\otimes p}$ is defined as in (1.8)-(1.9) and $\Phi_{p}(t, s ; x-y)$, defined in the obvious manner, depends only on the difference $x-y$. To see this dependency and to prepare for the future computations, we rewrite $\Phi_{p}(t, s ; x-y)$ using Fourier transform in space:

$$
\begin{aligned}
& \Phi_{p}(t, s ; x-y)=(p !)^{2}\left\langle f_{t, x, p}, \tilde{f}_{s, y, p}\right\rangle_{\mathcal{H} \otimes p} \\
& =p ! \sum_{\sigma \in \mathfrak{S}_{p}} \int_{\Delta_{p}(t)} d \boldsymbol{s}_{\boldsymbol{p}} \int_{[0, s]^{p}} d \tilde{\boldsymbol{s}}_{\boldsymbol{p}}\left(\prod_{j=1}^{p} \gamma_{0}\left(s_{j}-\tilde{\boldsymbol{s}}_{j}\right)\right)
\end{aligned}
$$




$$
\begin{aligned}
& \int_{\mathbb{R}^{2 p d}} d \boldsymbol{y}_{\boldsymbol{p}} d \tilde{\boldsymbol{y}}_{\boldsymbol{p}}\left(\prod_{j=1}^{p} \gamma\left(y_{j}-\tilde{y}_{j}\right)\right) \\
& \times\left(\prod_{j=0}^{p-1} G_{s_{j}-s_{j+1}}\left(y_{j}-y_{j+1}\right)\right)\left(\prod_{j=0}^{p-1} G_{\tilde{s}_{\sigma(j)}-\tilde{s}_{\sigma(j+1)}}\left(\tilde{y}_{\sigma(j)}-\tilde{y}_{\sigma(j+1)}\right)\right) \\
= & p ! \sum_{\sigma \in \mathfrak{S}_{p}} \int_{\Delta_{p}(t)} d \boldsymbol{s}_{\boldsymbol{p}} \int_{[0, s]^{p}} d \tilde{\boldsymbol{s}}_{\boldsymbol{p}}\left(\prod_{j=1}^{p} \gamma_{0}\left(s_{j}-\tilde{s}_{j}\right)\right) \\
& \times \int_{\mathbb{R}^{p d}} d \boldsymbol{\xi}_{\boldsymbol{p}}\left(\prod_{j=1}^{p} \varphi\left(\xi_{j}\right)\right) e^{-i(x-y) \cdot\left(\xi_{1}+\cdots+\xi_{p}\right)} \\
& \times\left(\prod_{j=0}^{p-1} \widehat{G}_{s_{j}-s_{j+1}}\left(\xi_{p}+\cdots+\xi_{j+1}\right)\right)\left(\prod_{j=0}^{p-1} \widehat{G}_{\tilde{s}_{\sigma(j)}-\tilde{s}_{\sigma(j+1)}}\left(\xi_{\sigma(p)}+\cdots+\xi_{\sigma(j+1)}\right)\right)
\end{aligned}
$$

where $\Delta_{p}(t)=\left\{\boldsymbol{s}_{\boldsymbol{p}}: t>s_{1}>\cdots>s_{p}>0\right\},\left(s_{0}, y_{0}, \tilde{s}_{\sigma(0)}, \tilde{y}_{\sigma(0)}\right)=(t, x, s, y)$, $\widehat{G}_{t}(\xi)=\frac{\sin (t|\xi|)}{|\xi|}$ is introduced in (2.29) and we have used again the convention $G_{t}(z)=0$ for $t \leq 0$.

Relation (4.6) shows that $\Phi_{p}(t, s ; x-y)$ is always nonnegative and equality (4.7) indicates that $\Phi_{p}(t, s ; x-y)$ indeed depends only on the difference $x-y$, so that we can write

$$
\Phi_{p}(t, s ; z)=(p !)^{2}\left\langle\widetilde{f}_{t, z, p}, \widetilde{f_{s, 0}}, p\right\rangle_{\mathcal{H} \otimes p}
$$

Note that $\Phi_{p}(t, t ; 0)$ coincides with $\alpha_{p}(t)$ given in [3, Equation (4.11)]. Moreover, applying Lemma 2.5 with $\mu_{p}\left(d \boldsymbol{\xi}_{\boldsymbol{p}}\right)=\varphi\left(\xi_{1}\right) \cdots \varphi\left(\xi_{p}\right) d \xi_{1} \cdots d \xi_{p} \quad$ and $g\left(s_{1}, \xi_{1}, \ldots, s_{p}, \xi_{p}\right)=\prod_{j=0}^{p-1}\left|\widehat{G}_{s_{j}-s_{j+1}}\left(\xi_{p}+\cdots+\xi_{j+1}\right)\right|$, we get (with $\left.s \leq t\right)$

$$
\Phi_{p}(t, s ; z) \leq \Gamma_{t}^{p} p ! \int_{\Delta_{p}(t)} d \boldsymbol{s}_{\boldsymbol{p}} \int_{\mathbb{R}_{p d}} \mu\left(d \boldsymbol{\xi}_{\boldsymbol{p}}\right) \prod_{j=0}^{p-1}\left|\widehat{G}_{s_{j}-s_{j+1}}\left(\xi_{p}+\cdots+\xi_{j+1}\right)\right|^{2},
$$

where we recall that $\Gamma_{t}=\int_{-t}^{t} \gamma_{0}(a) d a$ and point out that the right-hand side of (4.9) is finite by applying Lemma 2.6 with $z_{j}=\xi_{j+1}+\cdots+\xi_{p}$ and $z_{p}=0$.

Now we are ready to show (4.1).

Proof of (4.1) Let us begin with

$$
\frac{\mathbb{E}\left[F_{R}(t) F_{R}(s)\right]}{R^{d}}=\int_{B_{R}^{2}} d x d y \frac{\mathbb{E}[u(t, x) u(s, y)]-1}{R^{d}}
$$




$$
=\sum_{p \geq 1} \frac{\omega_{d}}{p !} \int_{\mathbb{R}^{d}} \frac{\operatorname{Leb}\left(B_{R} \cap B_{R}(-z)\right)}{\operatorname{Leb}\left(B_{R}\right)} \Phi_{p}(t, s ; z) d z
$$

where $\omega_{1}=2, \omega_{2}=\pi$ and $\operatorname{Leb}(A)$ stands for the Lebesgue measure of $A \subset \mathbb{R}^{d}$. We claim that

$$
\sum_{p \geq 1} \frac{1}{p !} \int_{\mathbb{R}^{d}} \Phi_{p}(t, s ; z) d z<\infty
$$

from which and the dominated convergence theorem we can deduce that

$$
\lim _{R \rightarrow \infty} R^{-d} \mathbb{E}\left[F_{R}(t) F_{R}(s)\right]=\omega_{d} \sum_{p \geq 1} \frac{1}{p !} \int_{\mathbb{R}^{d}} \Phi_{p}(t, s ; z) d z
$$

We remark that, by the monotone convergence theorem and the fact that $\Phi_{p}(t, s ; z) \geq$ 0 for all $z \in \mathbb{R}^{d}$, the claim (4.10) is equivalent to

$$
\sup _{\varepsilon>0} \sum_{p \geq 1} \frac{1}{p !} \int_{\mathbb{R}^{d}} \Phi_{p}(t, s ; z) e^{-\frac{\varepsilon}{2}|z|^{2}} d z<\infty
$$

Let us show the claim (4.12).

For $p=1$, by direct computations, we can perform integration with respect to $z, y, \tilde{y}$ (one by one in this order) to obtain

$$
\begin{aligned}
\int_{\mathbb{R}^{d}} & \Phi_{1}(t, s ; z) d z \\
= & \int_{\mathbb{R}^{d}}\left(\int_{0}^{t} d r \int_{0}^{s} d \tilde{r} \gamma_{0}(r-\tilde{r}) \int_{\mathbb{R}^{2 d}} d y d \tilde{y} G_{t-r}(y-z) G_{s-\tilde{r}}(\tilde{y}) \gamma(y-\tilde{y})\right) d z \\
= & \gamma\left(\mathbb{R}^{d}\right) \int_{0}^{t} \int_{0}^{s} \gamma_{0}(r-\tilde{r})(t-r)(s-\tilde{r}) d \tilde{r} d r \leq \gamma\left(\mathbb{R}^{d}\right) t^{3} \Gamma_{t}
\end{aligned}
$$

where $\int_{\mathbb{R}^{d}} \Phi_{1}(t, s ; z) d z>0$ due to the non-degeneracy assumption (1.17) on $\gamma_{0}$. This implies in particular that $\sigma_{R}(t)>0$ for large enough $R$.

Next we consider $p \geq 2$. Using the expression (4.7) and applying Fubini's theorem with the dominance condition (4.9), we can write

$$
\begin{aligned}
\mathcal{T}_{p, \varepsilon} & :=(2 \pi)^{-d} \int_{\mathbb{R}^{d}} \Phi_{p}(t, s ; z) e^{-\frac{\varepsilon}{2}|z|^{2}} d z \\
= & p ! \sum_{\sigma \in \mathfrak{S}_{p}} \int_{\Delta_{p}(t)} d \boldsymbol{s}_{\boldsymbol{p}} \int_{[0, s]^{p}} d \tilde{\boldsymbol{s}}_{\boldsymbol{p}} \prod_{j=1}^{p} \gamma_{0}\left(s_{j}-\tilde{s}_{j}\right) \int_{\mathbb{R}^{p d}} d \boldsymbol{\xi}_{\boldsymbol{p}} \\
& \times p_{\varepsilon}\left(\xi_{1}+\cdots+\xi_{p}\right) \prod_{j=0}^{p-1} \varphi\left(\xi_{j+1}\right) \widehat{G}_{s_{j}-s_{j+1}}\left(\xi_{p}+\cdots+\xi_{j+1}\right)
\end{aligned}
$$




$$
\begin{aligned}
& \widehat{G}_{\tilde{S}_{\sigma(j)}-\tilde{s}_{\sigma(j+1)}}\left(\xi_{\sigma(p)}+\cdots+\xi_{\sigma(j+1)}\right) \\
& \quad \leq \Gamma_{t}^{p} p ! \int_{\Delta_{p}(t)} d \boldsymbol{s}_{\boldsymbol{p}} \int_{\mathbb{R} p d} d \boldsymbol{\xi}_{\boldsymbol{p}}\left(\prod_{j=1}^{p} \varphi\left(\xi_{j}\right)\right) p_{\varepsilon}\left(\sum_{j=1}^{p} \xi_{j}\right) \\
& \quad \prod_{j=0}^{p-1}\left|\widehat{G}_{s_{j}-s_{j+1}}\left(\xi_{p}+\cdots+\xi_{j+1}\right)\right|^{2},
\end{aligned}
$$

where $p_{\varepsilon}(\xi)=(2 \pi \varepsilon)^{-d / 2} e^{-|\xi|^{2} /(2 \varepsilon)}$ for $\xi \in \mathbb{R}^{d}$ and we applied Lemma 2.5 with $\mu_{p}\left(d \boldsymbol{\xi}_{\boldsymbol{p}}\right)=\varphi\left(\xi_{1}\right) \cdots \varphi\left(\xi_{p}\right) p_{\varepsilon}\left(\xi_{1}+\cdots+\xi_{p}\right) d \xi_{1} \cdots d \xi_{p}$.

Next, we make the change of variables

$$
\eta_{j}=\xi_{p}+\cdots+\xi_{j} \text { with the convention } \eta_{p+1}=0
$$

and the bound (4.14) becomes

$$
\begin{aligned}
\mathcal{T}_{p, \varepsilon} \leq & \Gamma_{t}^{p} p ! \int_{\Delta_{p}(t)} d \boldsymbol{s}_{\boldsymbol{p}} \int_{\mathbb{R}^{p d}} d \boldsymbol{\eta}_{\boldsymbol{p}}\left(\prod_{j=1}^{p} \varphi\left(\eta_{j}-\eta_{j+1}\right)\right) p_{\varepsilon}\left(\eta_{1}\right) \prod_{j=0}^{p-1}\left|\widehat{G}_{s_{j}-s_{j+1}}\left(\eta_{j+1}\right)\right|^{2} \\
\leq & \Gamma_{t}^{p} p !\|\varphi\|_{\infty} t^{2} \int_{\mathbb{R}^{d}} d \eta_{1} p_{\varepsilon}\left(\eta_{1}\right) \int_{\Delta_{p}(t)} d \boldsymbol{s}_{\boldsymbol{p}} \int_{\mathbb{R}^{p d-d}} d \eta_{2} \cdots d \eta_{p} \\
& \left(\prod_{j=2}^{p} \varphi\left(\eta_{j}-\eta_{j+1}\right)\right)\left|\widehat{G}_{s_{1}-s_{2}}\left(\eta_{2}\right) \widehat{G}_{S_{2}-s_{3}}\left(\eta_{3}\right) \cdots \widehat{G}_{s_{p-1}-s_{p}}\left(\eta_{p}\right)\right|^{2} \\
& =\Gamma_{t}^{p} p !\|\varphi\|_{\infty} t^{2} \int_{\mathbb{R}^{d}} d \eta_{1} p_{\varepsilon}\left(\eta_{1}\right) Q_{p-1},
\end{aligned}
$$

where we used $\left|\widehat{G}_{t-s_{1}}(\xi)\right| \leq t$, and $\varphi\left(\eta_{1}-\eta_{2}\right) \leq\|\varphi\|_{\infty}$ (which is finite because $\left.\gamma\left(\mathbb{R}^{d}\right)<\infty\right)$ to obtain $(4.15)$, and

$$
Q_{p-1}:=\int_{\Delta_{p}(t)} d \boldsymbol{s}_{\boldsymbol{p}} \int_{\mathbb{R}^{p d-d}} \prod_{j=2}^{p} \varphi\left(\eta_{j}-\eta_{j+1}\right)\left|\widehat{G}_{s_{j-1}-s_{j}}\left(\eta_{j}\right)\right|^{2} d \eta_{j}
$$

Observe that $Q_{p-1}$ does not depend on $\eta_{1}$, thus for any $p \geq 2$

$$
\mathcal{T}_{p, \varepsilon} \leq \Gamma_{t}^{p} p !\|\varphi\|_{\infty} t^{2} Q_{p-1}
$$

By Lemma 2.6, we have for any $p \geq 2$

$$
Q_{p-1} \leq\left(2\left(t^{2} \vee 1\right) \int_{\mathbb{R}^{d}} \frac{\mu(d \xi)}{1+|\xi|^{2}}\right)^{p-1} \frac{t^{p}}{p !} \leq \frac{C^{p}}{p !}
$$


Now, plugging the above estimate and (4.17) into (4.12), and using (4.13) for $p=1$, we have

$\sup _{\varepsilon>0} \sum_{p \geq 1} \frac{1}{p !} \int_{\mathbb{R}^{d}} \Phi_{p}(t, s ; z) e^{-\frac{\varepsilon}{2}|z|^{2}} d z \leq \gamma\left(\mathbb{R}^{d}\right) t^{3} \Gamma_{t}+(2 \pi)^{d}\|\varphi\|_{\infty} t^{2} \sum_{p \geq 2} \frac{\Gamma_{t}^{p} C^{p}}{p !}<+\infty$

This shows the claim (4.12) and the claim (4.10), which confirm the limiting covariance structure (4.11). Hence the proof of (4.1) is completed.

\subsubsection{Proof of part (2) in Proposition 4.1}

In this case, the corresponding spectral density is given by $\varphi(\xi)=c_{d, \beta}|\xi|^{\beta-d}$, for some constant $c_{d, \beta}$ that only depends on $d$ and $\beta$.

Now, let us recall the chaos expansion (1.7) of $u(t, x)$, from which we can obtain the following chaos expansion of $F_{R}(t)$ :

$$
F_{R}(t)=\sum_{p \geq 1} \mathbf{J}_{p, R}(t)
$$

where $\mathbf{J}_{p, R}(t):=I_{p}\left(\int_{|x| \leq R} \tilde{f}_{t, x, p} d x\right)$ is the projection of $F_{R}(t)$ onto the $p$ th Wiener chaos, with $\tilde{f}_{t, x, p}$ given as in (1.9).

Using the orthogonality of Wiener chaoses with different order, we have

$$
\sigma_{R}^{2}(t)=\operatorname{Var}\left(F_{R}(t)\right)=\sum_{p \geq 1} \operatorname{Var}\left(\mathbf{J}_{p, R}(t)\right)
$$

Let us first consider the variance of $\mathbf{J}_{1, R}(t)$. With $B_{R}=\left\{x \in \mathbb{R}^{d}:|x| \leq R\right\}$, we can write

$$
\begin{aligned}
& \operatorname{Var}\left(\mathbf{J}_{1, R}(t)\right)=\int_{B_{R}^{2}} d x d x^{\prime}\left\langle G_{t-\bullet}(x-*), G_{t-\bullet}\left(x^{\prime}-*\right)\right\rangle_{\mathcal{H}} \\
& \quad=\int_{B_{R}^{2}} d x d x^{\prime} \int_{[0, t]^{2}} d s d s^{\prime} \gamma_{0}\left(s-s^{\prime}\right) \int_{\mathbb{R}^{d}} d \xi \varphi(\xi) e^{-i\left(x-x^{\prime}\right) \cdot \xi} \widehat{G}_{t-s}(\xi) \widehat{G}_{t-s^{\prime}}(\xi)
\end{aligned}
$$

Then, making the change of variables $\left(x, x^{\prime}, \xi\right) \rightarrow\left(R x, R x^{\prime}, \xi / R\right)$, we get

$$
\begin{gathered}
\operatorname{Var}\left(\mathbf{J}_{1, R}(t)\right)=R^{2 d-\beta} \int_{[0, t]^{2}} d s d s^{\prime} \gamma_{0}\left(s-s^{\prime}\right) \int_{B_{1}^{2}} d x d x^{\prime} \\
\int_{\mathbb{R}^{d}} d \xi \varphi(\xi) e^{-i\left(x-x^{\prime}\right) \cdot \xi} \widehat{G}_{t-s}(\xi / R) \widehat{G}_{t-s^{\prime}}(\xi / R) .
\end{gathered}
$$


Note that $\widehat{G}_{t}(\xi / R)$ is uniformly bounded and convergent to $t$ as $R \rightarrow \infty$; observe also that

$$
\ell_{R}(\xi):=\int_{B_{R}^{2}} d x d x^{\prime} e^{-i\left(x-x^{\prime}\right) \cdot \xi}=\left|\mathcal{F} \mathbf{1}_{B_{R}}\right|^{2}(\xi) \in[0, \infty)
$$

Thus we deduce from the dominated convergence theorem that, with $\kappa_{\beta, d}:=$ $\int_{B_{1}^{2}} d x d x^{\prime}\left|x-x^{\prime}\right|^{-\beta}$

$$
\begin{aligned}
& \frac{\operatorname{Var}\left(\mathbf{J}_{1, R}(t)\right)}{R^{2 d-\beta}} \stackrel{R \rightarrow \infty}{\longrightarrow} \int_{[0, t]^{2}} d s d s^{\prime} \gamma_{0}\left(s-s^{\prime}\right)(t-s)\left(t-s^{\prime}\right) \int_{\mathbb{R}^{d}} d \xi \varphi(\xi)\left|\mathcal{F} \mathbf{1}_{B_{1}}\right|^{2}(\xi) \\
& \quad=\kappa_{\beta, d} \int_{[0, t]^{2}} d s d s^{\prime} \gamma_{0}\left(s-s^{\prime}\right) s s^{\prime}
\end{aligned}
$$

In the same way, we can get

$$
\frac{\mathbb{E}\left[\mathbf{J}_{1, R}(t) \mathbf{J}_{1, R}(s)\right]}{R^{2 d-\beta}} \stackrel{R \rightarrow \infty}{\longrightarrow} \kappa_{\beta, d} \int_{0}^{t} d r \int_{0}^{s} d r^{\prime} \gamma_{0}\left(r-r^{\prime}\right)(t-r)\left(s-r^{\prime}\right)
$$

In what follows, we will show that as $R \rightarrow \infty$,

$$
\sum_{p \geq 2} \operatorname{Var}\left(\mathbf{J}_{p, R}(t)\right)=o\left(R^{2 d-\beta}\right) .
$$

In view of the orthogonality again, the above claim (4.22) and the results (4.20)-(4.21) imply that the first chaos of $F_{R}(t)$ is dominant and

$$
\frac{\mathbb{E}\left[F_{R}(t) F_{R}(s)\right]}{R^{2 d-\beta}} \stackrel{R \rightarrow \infty}{\longrightarrow} \kappa_{\beta, d} \int_{0}^{t} d r \int_{0}^{s} d r^{\prime} \gamma_{0}\left(r-r^{\prime}\right)(t-r)\left(s-r^{\prime}\right),
$$

which gives us the desired limiting covariance structure. Moreover, we obtain immediately that the process $\left\{R^{-d+\frac{\beta}{2}} F_{R}(t): t \in \mathbb{R}_{+}\right\}$converges in finite-dimensional distributions to the centered Gaussian process $\mathcal{G}_{\beta}$, whose covariance structure is given by (1.19).

The rest of Sect. 4.1.2 is then devoted to proving (4.22). We point out that the strategy in Sect. 4.1.1 can not be directly used, because $\varphi$ is not uniformly bounded here.

Proof of Claim (4.22) We begin by writing (with $s_{0}=\tilde{s}_{\sigma(0)}=t$ and $B_{R}=\{x:|x| \leq$ $R\})$

$$
\begin{aligned}
& \operatorname{Var}\left(\mathbf{J}_{p, R}(t)\right)=p ! \int_{B_{R}^{2}} d x d x^{\prime}\left\langle\tilde{f}_{t, x, p}, \tilde{f}_{t, x^{\prime}, p}\right\rangle_{\mathcal{H}^{\otimes p}}=p ! \int_{B_{R}^{2}} d x d x^{\prime}\left\langle f_{t, x, p}, \widetilde{f}_{t, x^{\prime}, p}\right\rangle_{\mathcal{H}^{\otimes p}} \\
& \quad=c_{d, \beta}^{p} \sum_{\sigma \in \mathfrak{S}_{p}} \int_{B_{R}^{2}} d x d x^{\prime} \int_{[0, t]^{2 p}} d \boldsymbol{s}_{\boldsymbol{p}} d \tilde{\boldsymbol{s}}_{\boldsymbol{p}} \prod_{k=1}^{p} \gamma_{0}\left(s_{k}-\tilde{s}_{k}\right) \int_{\mathbb{R}^{p d}}\left(\prod_{j=1}^{p} d \xi_{j}\left|\xi_{j}\right|^{\beta-d}\right)
\end{aligned}
$$




$$
\begin{aligned}
& \times e^{-i\left(x-x^{\prime}\right) \cdot\left(\xi_{p}+\cdots+\xi_{1}\right)} \prod_{j=0}^{p-1} \widehat{G}_{s_{j}-s_{j+1}}\left(\xi_{p}+\cdots+\xi_{j+1}\right) \widehat{G}_{\tilde{s}_{\sigma(j)}-\tilde{s}_{\sigma(j+1)}} \\
& \times\left(\xi_{\sigma(p)}+\cdots+\xi_{\sigma(j+1)}\right),
\end{aligned}
$$

where we recall the convention that $G_{t}(z)=0$ for $t \leq 0$.

Then, recalling definition (4.19) of $\ell_{R}(\xi)$, we can apply Lemma 2.5 with

$$
\mu\left(d \boldsymbol{\xi}_{\boldsymbol{p}}\right)=\varphi\left(\xi_{1}\right) \cdots \varphi\left(\xi_{p}\right) \ell_{R}\left(\xi_{1}+\cdots+\xi_{p}\right) d \xi_{1} \cdots d \xi_{p}
$$

to get $\operatorname{Var}\left(\mathbf{J}_{p, R}(t)\right)$ bounded by

$$
\begin{aligned}
& c_{d, \beta}^{p} \Gamma_{t}^{p} \int_{\Delta_{p}(t)} d \boldsymbol{s}_{\boldsymbol{p}} \int_{\mathbb{R}^{p d}}\left(\prod_{j=1}^{p} d \xi_{j}\left|\xi_{j}\right|^{\beta-d}\right) \ell_{R}\left(\xi_{1}+\cdots+\xi_{p}\right) \\
& \quad \times \prod_{j=0}^{p-1}\left|\widehat{G}_{s_{j}-s_{j+1}}\left(\xi_{p}+\cdots+\xi_{j+1}\right)\right|^{2} .
\end{aligned}
$$

Making change of variables

(i) $\eta_{j}=\xi_{p}+\cdots+\xi_{j}$ with $\eta_{p+1}=0 \quad$ (ii) $\left(x, x^{\prime}, \eta_{1}\right) \rightarrow\left(R x, R x^{\prime}, \eta_{1} R^{-1}\right)$,

we obtain

$$
\begin{aligned}
\operatorname{Var}\left(\mathbf{J}_{p, R}(t)\right) \leq & c_{d, \beta}^{p} \Gamma_{t}^{p} \int_{\Delta_{p}(t)} d \boldsymbol{s}_{\boldsymbol{p}} \int_{\mathbb{R}^{p d}}\left(\prod_{j=1}^{p} d \eta_{j}\left|\eta_{j}-\eta_{j+1}\right|^{\beta-d}\right) \\
& \times\left(\int_{B_{R}^{2}} d x d x^{\prime} e^{-i\left(x-x^{\prime}\right) \cdot \eta_{1}}\right) \prod_{j=0}^{p-1}\left|\widehat{G}_{s_{j}-s_{j+1}}\left(\eta_{j+1}\right)\right|^{2} \\
= & c_{d, \beta}^{p} \Gamma_{t}^{p} R^{2 d-\beta} \int_{\Delta_{p}(t)} d \boldsymbol{s}_{\boldsymbol{p}} \int_{\mathbb{R}^{p d}} d \eta_{1}\left|\eta_{1}-\eta_{2} R\right|^{\beta-d} \\
& \left(\prod_{j=2}^{p} d \eta_{j}\left|\eta_{j}-\eta_{j+1}\right|^{\beta-d}\right) \\
& \times\left(\int_{B_{1}^{2}} d x d x^{\prime} e^{-i\left(x-x^{\prime}\right) \cdot \eta_{1}}\right)\left|\widehat{G}_{t-s_{1}}\left(\eta_{1} / R\right)\right|^{2} \prod_{j=1}^{p-1}\left|\widehat{G}_{s_{j}-s_{j+1}}\left(\eta_{j+1}\right)\right|^{2} \\
\leq & t^{2} c_{d, \beta}^{p-1} \Gamma_{t}^{p} R^{2 d-\beta} \int_{\Delta_{p}(t)} d \boldsymbol{s}_{\boldsymbol{p}} \int_{\mathbb{R}^{p d-d}}\left(\prod_{j=2}^{p} d \eta_{j}\left|\eta_{j}-\eta_{j+1}\right|^{\beta-d}\right)^{p-1} \mid \widehat{G}_{s_{j}-\left.s_{j+1}\left(\eta_{j+1}\right)\right|^{2}} \\
& \times\left(\int_{B_{1}^{2}} d x d x^{\prime}\left|x-x^{\prime}\right|^{-\beta} e^{-i\left(x-x^{\prime}\right) \cdot \eta_{2} R} \prod_{j=1}^{p}\right.
\end{aligned}
$$


where in the last inequality we used $\left|\widehat{G}_{t}\right| \leq t$ and the following Fourier transform:

$$
\begin{aligned}
& \int_{B_{1}^{2}} d x d x^{\prime} c_{d, \beta} \int_{\mathbb{R}^{d}} d \eta_{1}\left|\eta_{1}-\eta_{2} R\right|^{\beta-d} e^{-i\left(x-x^{\prime}\right) \cdot \eta_{1}} \\
& =c_{d, \beta} \int_{\mathbb{R}^{d}} d \eta_{1}\left|\eta_{1}-\eta_{2} R\right|^{\beta-d}\left|\mathcal{F} \mathbf{1}_{B_{1}}\right|^{2}\left(\eta_{1}\right) \\
& =\int_{B_{1}^{2}} d x d x^{\prime}\left|x-x^{\prime}\right|^{-\beta} e^{-i\left(x-x^{\prime}\right) \cdot \eta_{2} R} .
\end{aligned}
$$

Note that the integral $\int_{B_{1}^{2}} d x d x^{\prime}\left|x-x^{\prime}\right|^{-\beta} e^{-i\left(x-x^{\prime}\right) \cdot \eta_{2} R}$ is uniformly bounded by $\kappa_{\beta, d}$ and it converges to zero as $R \rightarrow \infty$ for $\eta_{2} \neq 0$. This convergence is a consequence of the Riemann-Lebesgue's lemma. Taking into account the definition (4.16) of $Q_{p-1}$, then we have

$$
R^{\beta-2 d} \operatorname{Var}\left(\mathbf{J}_{p, R}(t)\right) \leq t^{2} \kappa_{\beta, d} \Gamma_{t}^{p} Q_{p-1},
$$

which is summable over $p \geq 2$ by the arguments in the previous section. Hence by the dominated convergence theorem, we get

$$
R^{\beta-2 d} \sum_{p \geq 2} \operatorname{Var}\left(\mathbf{J}_{p, R}(t)\right) \stackrel{R \rightarrow \infty}{\longrightarrow} 0 .
$$

This proves the claim (4.22).

\subsubsection{Proof of part (3) in Proposition 4.1}

Recall the two cases from (4.3):

$$
\begin{cases}\left(c_{1}\right) & \gamma_{i}\left(x_{i}\right)=\left|x_{i}\right|^{-\beta_{i}} \text { for some } \beta_{i} \in(0,1), i=1,2, \\ \left(c_{2}\right) & \gamma_{1} \in L^{1}(\mathbb{R}) \text { and } \gamma_{2}(x)=|x|^{-\beta} \text { for some } \beta \in(0,1) .\end{cases}
$$

In $\left(c_{1}\right)$, the spectral density is $\varphi\left(\xi_{1}, \xi_{2}\right)=c_{1, \beta_{1}} c_{1, \beta_{2}}\left|\xi_{1}\right|^{\beta_{1}-1}\left|\xi_{2}\right|^{\beta_{2}-1}$ for $\left(\xi_{1}, \xi_{2}\right) \in$ $\mathbb{R}^{2}$, where $c_{1, \beta}$ is a constant that only depends on $\beta$. Now, using the notation from Sect. 4.1.2, we write

$$
\begin{aligned}
& \operatorname{Var}\left(\mathbf{J}_{1, R}(t)\right)=\int_{B_{R}^{2}} d x d x^{\prime} \int_{[0, t]^{2}} d s d s^{\prime} \gamma_{0}\left(s-s^{\prime}\right) \\
& \quad \times \int_{\mathbb{R}^{d}} d \xi \varphi(\xi) e^{-i\left(x-x^{\prime}\right) \cdot \xi} \widehat{G}_{t-s}(\xi) \widehat{G}_{t-s^{\prime}}(\xi) \quad \text { see }(4.18) \\
& =R^{4-\beta_{1}-\beta_{2}} \int_{[0, t]^{2}} d s d s^{\prime} \gamma_{0}\left(s-s^{\prime}\right) \int_{\mathbb{R}^{d}} d \xi \varphi\left(\xi_{1}, \xi_{2}\right) \\
& \quad \times \int_{B_{1}^{2}} d x d x^{\prime} e^{-i\left(x-x^{\prime}\right) \cdot \xi} \widehat{G}_{t-s}(\xi / R) \widehat{G}_{t-s^{\prime}}(\xi / R)
\end{aligned}
$$


where the last equality is obtained by the change of variables $\left(x, x^{\prime}, \xi_{1}, \xi_{2}\right)$ to $\left(R x, R x^{\prime}, \xi_{1} / R, \xi_{2} / R\right)$. Thus, by the exactly same arguments that lead to (4.20), we can get

$$
\frac{\operatorname{Var}\left(\mathbf{J}_{1, R}(t)\right)}{R^{4-\beta_{1}-\beta_{2}}} \stackrel{R \rightarrow \infty}{\longrightarrow} K_{\beta_{1}, \beta_{2}} \int_{[0, t]^{2}} d s d s^{\prime} \gamma_{0}\left(s-s^{\prime}\right) s s^{\prime}
$$

with $K_{\beta_{1}, \beta_{2}}$ introduced in (1.22). Similar to (4.21), we also have

$$
\frac{\mathbb{E}\left[\mathbf{J}_{1, R}(t) \mathbf{J}_{1, R}(s)\right]}{R^{4-\beta_{1}-\beta_{2}}} \stackrel{R \rightarrow \infty}{\longrightarrow} K_{\beta_{1}, \beta_{2}} \int_{0}^{t} d r \int_{0}^{s} d r^{\prime} \gamma_{0}\left(r-r^{\prime}\right)(t-r)\left(s-r^{\prime}\right) .
$$

To obtain the result $\left(r_{1}\right)$, it remains to show

$$
\sum_{p \geq 2} \operatorname{Var}\left(\mathbf{J}_{p, R}(t)\right)=o\left(R^{4-\beta_{1}-\beta_{2}}\right)
$$

Its proof can be done verbatim as for the result (4.22), so we omit the details here.

Finally, let us look at the more interesting case $\left(c_{2}\right)$ where $\gamma_{1} \in L^{1}(\mathbb{R})$ and $\gamma_{2}(x)=$ $|x|^{-\beta}$ for some fixed $\beta \in(0,1)$. In this case, the corresponding spectral density is $\varphi\left(\xi_{1}, \xi_{2}\right)=\varphi_{1}\left(\xi_{1}\right) \varphi_{2}\left(\xi_{2}\right)$, where

$\left\{\begin{array}{l}\text { (i) } \gamma_{1}=\mathcal{F} \varphi_{1} \text { and } \varphi_{1} \text { is uniformly continuous and bounded, } \\ \text { (ii) } \varphi_{2}\left(\xi_{2}\right)=c_{1, \beta}\left|\xi_{2}\right|^{\beta-1} \text { for some constant } c_{1, \beta} \text { that only depends on } \beta\end{array}\right.$

Let us begin with (4.18) and make the usual change of variables $\left(x, x^{\prime}, \xi\right) \rightarrow$ $\left(R x, R x^{\prime}, \xi / R\right)$ to obtain

$$
\begin{aligned}
& \operatorname{Var}\left(\mathbf{J}_{1, R}(t)\right)=\int_{B_{R}^{2}} d x d x^{\prime} \int_{[0, t]^{2}} d s d s^{\prime} \gamma_{0}\left(s-s^{\prime}\right) \\
& \int_{\mathbb{R}^{2}} d \xi \varphi_{1}\left(\xi_{1}\right) \varphi_{2}\left(\xi_{2}\right) e^{-i\left(x-x^{\prime}\right) \cdot \xi} \widehat{G}_{t-s}(\xi) \widehat{G}_{t-s^{\prime}}(\xi) \\
& =R^{3-\beta} \int_{[0, t]^{2}} d s d s^{\prime} \gamma_{0}\left(s-s^{\prime}\right) \int_{\mathbb{R}^{2}} d \xi \varphi_{1}\left(\xi_{1} / R\right) \varphi_{2}\left(\xi_{2}\right) \\
& \left(\int_{B_{1}^{2}} d x d x^{\prime} e^{-i\left(x-x^{\prime}\right) \cdot \xi}\right) \widehat{G}_{t-s}(\xi / R) \widehat{G}_{t-s^{\prime}}(\xi / R) \\
& =R^{3-\beta} \int_{[0, t]^{2}} d s d s^{\prime} \gamma_{0}\left(s-s^{\prime}\right) \\
& \int_{\mathbb{R}^{2}} d \xi \varphi_{1}\left(\xi_{1} / R\right) \varphi_{2}\left(\xi_{2}\right)\left|\mathcal{F} \mathbf{1}_{B_{1}}\right|^{2}(\xi) \widehat{G}_{t-s}(\xi / R) \widehat{G}_{t-s^{\prime}}(\xi / R) .
\end{aligned}
$$


Recall that $\varphi_{1}, \widehat{G}_{t-s}$ and $\widehat{G}_{t-s^{\prime}}$ are uniformly bounded and continuous. Note that, applying Plancherel's theorem and the Parseval-type relation (2.3), we have

$$
\begin{aligned}
\int_{\mathbb{R}^{2}} d \xi \varphi_{2}\left(\xi_{2}\right)\left|\mathcal{F} \mathbf{1}_{B_{1}}\right|^{2}(\xi) & =2 \pi \int_{\mathbb{R}^{2}} d x_{1} d \xi_{2} \varphi_{2}\left(\xi_{2}\right)\left|\mathcal{F} \mathbf{1}_{B_{1}}\left(x_{1}, \bullet\right)\left(\xi_{2}\right)\right|^{2} \\
& =2 \pi \int_{\mathbb{R}^{3}} d x_{1} d x_{2} d x_{3} \mathbf{1}_{\left\{x_{1}^{2}+x_{2}^{2} \leq 1\right\}} \mathbf{1}_{\left\{x_{1}^{2}+x_{3}^{2} \leq 1\right\}}\left|x_{2}-x_{3}\right|^{-\beta}<\infty
\end{aligned}
$$

Therefore, by the dominated convergence theorem and the fact that $\varphi_{1}(0)=\frac{1}{2 \pi} \gamma_{1}(\mathbb{R})$, we get

$$
\begin{aligned}
\frac{\operatorname{Var}\left(\mathbf{J}_{1, R}(t)\right)}{R^{3-\beta}} & \stackrel{R \rightarrow \infty}{\longrightarrow} \varphi_{1}(0) \int_{[0, t]^{2}} d s d s^{\prime} \gamma_{0}\left(s-s^{\prime}\right)(t-s)\left(t-s^{\prime}\right) \\
& \times \int_{\mathbb{R}^{2}} d \xi \varphi_{2}\left(\xi_{2}\right)\left|\mathcal{F} \mathbf{1}_{B_{1}}\right|^{2}(\xi) \\
& =\gamma_{1}(\mathbb{R}) \mathcal{L}_{\beta} \int_{[0, t]^{2}} d s d s^{\prime} \gamma_{0}\left(s-s^{\prime}\right) s s^{\prime}
\end{aligned}
$$

where $\mathcal{L}_{\beta}$ is defined in (1.24). In the same way, we get for $s, t \in(0, \infty)$,

$$
\frac{\mathbb{E}\left[\mathbf{J}_{1, R}(t) \mathbf{J}_{1, R}(s)\right]}{R^{3-\beta}} \stackrel{R \rightarrow \infty}{\longrightarrow} \gamma_{1}(\mathbb{R}) \mathcal{L}_{\beta} \int_{0}^{t} d r \int_{0}^{s} d r^{\prime} \gamma_{0}\left(r-r^{\prime}\right)(t-r)\left(s-r^{\prime}\right) .
$$

Now we claim that the other chaoses are negligible, that is, as $R \rightarrow \infty$,

$$
\sum_{p \geq 2} \operatorname{Var}\left(\mathbf{J}_{p, R}(t)\right)=o\left(R^{3-\beta}\right)
$$

Note that the desired limiting covariance structure follows from (4.27) and the above claim (4.28). The rest of this section is devoted to proving claim (4.28).

Proof of Claim (4.28) By the same arguments that lead to the estimate (4.23), we can obtain

$$
\begin{aligned}
& \operatorname{Var}\left(\mathbf{J}_{p, R}(t)\right) \leq \Gamma_{t}^{p} \int_{\Delta_{p}(t)} d \boldsymbol{s}_{\boldsymbol{p}} \int_{\mathbb{R}^{2 p}} d \boldsymbol{\xi}_{\boldsymbol{p}} \varphi_{p}\left(\boldsymbol{\xi}_{\boldsymbol{p}}\right) \\
& \quad \times \prod_{j=0}^{p-1}\left|\widehat{G}_{s_{j}-s_{j+1}}\left(\xi_{p}+\cdots+\xi_{j+1}\right)\right|^{2} \text { with } s_{0}=t,
\end{aligned}
$$

where $\varphi_{p}\left(\boldsymbol{\xi}_{\boldsymbol{p}}\right)=\varphi\left(\xi_{1}\right) \cdots \varphi\left(\xi_{p}\right) \ell_{R}\left(\xi_{1}+\cdots+\xi_{p}\right)$ for $\xi_{j}=\left(\xi_{j}^{(1)}, \xi_{j}^{(2)}\right) \in \mathbb{R}^{2}$, $j=1, \ldots, p$ and $\ell_{R}$ is defined in (4.19). Recall that in the current case, $\varphi(\xi)=$ 
$\varphi_{1}\left(\xi^{(1)}\right) \varphi_{2}\left(\xi^{(2)}\right)$ for $\xi=\left(\xi^{(1)}, \xi^{(2)}\right) \in \mathbb{R}^{2}$ and $\varphi_{1}, \varphi_{2}$ satisfy the conditions in (4.26). Then, the following change of variables

$$
\eta_{j}=\xi_{j}+\xi_{j+1}+\cdots+\xi_{p} \text { with } \eta_{p+1}=0
$$

yields

$$
\operatorname{Var}\left(\mathbf{J}_{p, R}(t)\right) \leq \Gamma_{t}^{p} \int_{\Delta_{p}(t)} d \boldsymbol{s}_{\boldsymbol{p}} \int_{\mathbb{R}^{2 p}} d \boldsymbol{\eta}_{\boldsymbol{p}} \ell_{R}\left(\eta_{1}\right) \prod_{j=0}^{p-1} \varphi\left(\eta_{j+1}-\eta_{j+2}\right)\left|\widehat{G}_{s_{j}-s_{j+1}}\left(\eta_{j+1}\right)\right|^{2} .
$$

In view of (4.19), we have $\ell_{R}\left(\eta_{1} / R\right)=R^{4} \ell_{1}\left(\eta_{1}\right)$. Thus, by changing $\eta_{1}$ to $\eta_{1} / R$, we write

$$
\begin{aligned}
\operatorname{Var}\left(\mathbf{J}_{p, R}(t)\right) \leq & R^{2} \Gamma_{t}^{p} \int_{\Delta_{p}(t)} d \boldsymbol{s}_{\boldsymbol{p}} \int_{\mathbb{R}^{2 p}} d \boldsymbol{\eta}_{\boldsymbol{p}} \ell_{1}\left(\eta_{1}\right) \varphi\left(\eta_{1} R^{-1}-\eta_{2}\right)\left|\widehat{G}_{t-s_{1}}\left(\eta_{1} / R\right)\right|^{2} \\
& \times \prod_{j=1}^{p-1} \varphi\left(\eta_{j+1}-\eta_{j+2}\right)\left|\widehat{G}_{s_{j}-s_{j+1}}\left(\eta_{j+1}\right)\right|^{2} \\
\leq & R^{3-\beta} \Gamma_{t}^{p}\left\|\varphi_{1}\right\|_{\infty} t^{2} \int_{\Delta_{p}(t)} d \boldsymbol{s}_{\boldsymbol{p}} \int_{\mathbb{R}^{2 p-2}} d \eta_{2} \ldots d \eta_{p} \\
& \left(\int_{\mathbb{R}^{2}} d \eta_{1} \ell_{1}\left(\eta_{1}\right) c_{1, \beta}\left|\eta_{1}^{(2)}-\eta_{2}^{(2)} R\right|^{\beta-1}\right) \\
& \times \prod_{j=1}^{p-1} \varphi\left(\eta_{j+1}-\eta_{j+2}\right)\left|\widehat{G}_{s_{j}-s_{j+1}}\left(\eta_{j+1}\right)\right|^{2}
\end{aligned}
$$

where we used $\left|\widehat{G}_{t-s_{1}}\left(\eta_{1} / R\right)\right|^{2} \leq t^{2}$. Observe that with $\eta=\left(\eta^{(1)}, \eta^{(2)}\right)$, we deduce from the fact $\ell_{1}(\eta)=\left|\mathcal{F} \mathbf{1}_{B_{1}}\right|^{2}\left(\eta^{(1)}, \eta^{(2)}\right)$ that

$$
\begin{aligned}
\int_{\mathbb{R}^{2}} d \eta \ell_{1}(\eta) \varphi_{2}\left(\eta^{(2)}-x R\right)= & \int_{\mathbb{R}^{2}} d \eta^{(1)} d \eta^{(2)}\left|\mathcal{F} \mathbf{1}_{B_{1}}\right|^{2}\left(\eta^{(1)}, \eta^{(2)}+x R\right) \varphi_{2}\left(\eta^{(2)}\right) \\
= & 2 \pi \int_{\mathbb{R}^{3}} \mathbf{1}_{\left\{x_{1}^{2}+x_{2}^{2} \leq 1\right\}} \mathbf{1}_{\left\{x_{1}^{2}+x_{3}^{2} \leq 1\right\}} \\
& e^{-i\left(x_{2}-x_{3}\right) x R}\left|x_{2}-x_{3}\right|^{-\beta} d x_{1} d x_{2} d x_{3},
\end{aligned}
$$

by inverting the Fourier transform. The above quantity is uniformly bounded by $2 \pi \mathcal{L}_{\beta}$ with $\mathcal{L}_{\beta}$ given in (1.24) and convergent to zero as $R \rightarrow \infty$ for every $x \neq 0$ in view of the Riemann-Lebesgue lemma. Thus, $R^{\beta-3} \operatorname{Var}\left(\mathbf{J}_{p, R}(t)\right)$ is uniformly bounded by $2 \pi \mathcal{L}_{\beta} \Gamma_{t}^{p}\left\|\varphi_{1}\right\|_{\infty} t^{2} Q_{p-1}$, with $Q_{p-1}$ given by (4.16) and it converges to zero as $R \rightarrow \infty$. Since $Q_{p} \leq C^{p} / p$ !, we have

$$
\sum_{p \geq 2} \Gamma_{t}^{p} Q_{p-1}<\infty
$$


and the dominated convergence theorem implies (4.28).

Remark 4.2 Under the assumptions of Proposition 4.1, we point out that $\sigma_{R}(t)>0$ for large enough $R$ so that the renormalized random variable $F_{R}(t) / \sigma_{R}(t)$ is well-defined for large $R$.

\subsection{Quantitative central limit theorems (QCLT) and f.d.d. convergence}

In this section, we prove the quantitative CLTs that are stated in Theorem 1.4 and, as an easy consequence, we are also able to show the convergence of finite-dimensional distributions in Theorem 1.4. We consider first the part (1) and later we treat parts (2) and (3).

\subsubsection{Part (1)}

We will first show the estimate

$$
d_{\mathrm{TV}}\left(F_{R}(t) / \sigma_{R}(t), Z\right) \lesssim R^{-d / 2}
$$

where $Z \sim N(0,1)$. By Proposition 1.8 applied to $\frac{1}{\sigma_{R}(t)} F_{R}(t)$, we have

$$
d_{\mathrm{TV}}\left(F_{R}(t) / \sigma_{R}(t), Z\right) \leq \frac{4}{\sigma_{R}^{2}(t)} \sqrt{\mathcal{A}_{R}}
$$

where

$$
\begin{aligned}
\mathcal{A}_{R}= & \int_{\mathbb{R}_{+}^{6} \times \mathbb{R}^{6 d}} d r d r^{\prime} d s d s^{\prime} d \theta d \theta^{\prime} d z d z^{\prime} d y d y^{\prime} d w d w^{\prime} \\
& \gamma_{0}\left(\theta-\theta^{\prime}\right) \gamma_{0}\left(s-s^{\prime}\right) \gamma_{0}\left(r-r^{\prime}\right) \gamma\left(z-z^{\prime}\right) \gamma\left(w-w^{\prime}\right) \\
& \times \gamma\left(y-y^{\prime}\right)\left\|D_{r, z} D_{\theta, w} F_{R}(t)\right\|_{4}\left\|D_{s, y} D_{\theta^{\prime}, w^{\prime}} F_{R}(t)\right\|_{4}\left\|D_{r^{\prime}, z^{\prime}} F_{R}(t)\right\|_{4} \| \\
& D_{s^{\prime}, y^{\prime}} F_{R}(t) \|_{4} .
\end{aligned}
$$

Recall from Sect. 4.1.1 that $\sigma_{R}^{2}(t) \sim R^{d}$. Therefore, in order to show (4.29) it suffices to prove the estimate

$$
\mathcal{A}_{R} \lesssim R^{d}
$$

Using Minkowski's inequality, we can write

$$
\left\|D_{r, z} D_{\theta, w} F_{R}(t)\right\|_{4}=\left\|\int_{B_{R}} D_{r, z} D_{\theta, w} u(t, x) d x\right\|_{4} \leq \int_{B_{R}}\left\|D_{r, z} D_{\theta, w} u(t, x)\right\|_{4} d x .
$$

Then, it follows from our fundamental estimates in Theorem 1.3 that

$$
\left\|D_{r, z} D_{\theta, w} F_{R}(t)\right\|_{4} \lesssim \int_{B_{R}} \widetilde{f}_{t, x, 2}(r, z, \theta, w) d x
$$


with

$$
\begin{aligned}
& \tilde{f}_{t, x, 2}(r, z, \theta, w) \\
& \quad=\frac{1}{2}\left[G_{t-r}(x-z) G_{r-\theta}(z-w) \mathbf{1}_{\{r>\theta\}}+G_{t-\theta}(x-w) G_{\theta-r}(z-w) \mathbf{1}_{\{r<\theta\}}\right]
\end{aligned}
$$

and, in the same way, we have

$$
\left\|D_{r, z} F_{R}(t)\right\|_{4} \lesssim \int_{B_{R}} G_{t-r}(x-z) d x
$$

where the implicit constants in (4.32)-(4.33) do not depend on $(R, r, z, \theta, w)$ and are increasing in $t$. Now, plugging (4.32)-(4.33) into the expression of $\mathcal{A}_{R}$, we get

$$
\begin{aligned}
\mathcal{A}_{R} & \lesssim \int_{[0, t]^{6} \times \mathbb{R}^{6 d}} d r d r^{\prime} d s d s^{\prime} d \theta d \theta^{\prime} d z d z^{\prime} d y d y^{\prime} d w d w^{\prime} \\
& \times \gamma_{0}\left(r-r^{\prime}\right) \gamma_{0}\left(s-s^{\prime}\right) \gamma_{0}\left(\theta-\theta^{\prime}\right) \gamma\left(z-z^{\prime}\right) \gamma\left(w-w^{\prime}\right) \\
& \times \gamma\left(y-y^{\prime}\right) \int_{B_{R}^{4}} \widetilde{f}_{t, x_{1}, 2}(r, z, \theta, w) \widetilde{f}_{t, x_{2}, 2}\left(s, y, \theta^{\prime}, w^{\prime}\right) G_{t-r^{\prime}}\left(x_{3}-z^{\prime}\right) \\
& \times G_{t-s^{\prime}}\left(x_{4}-y^{\prime}\right) d \boldsymbol{x}_{\mathbf{4}}=: \sum_{j=1}^{4} \mathcal{A}_{R, j} .
\end{aligned}
$$

The four terms $\mathcal{A}_{R, 1}, \ldots, \mathcal{A}_{R, 4}$ are defined according to whether $r>\theta$ or $r<\theta$, and whether $s>\theta^{\prime}$ or $s<\theta^{\prime}$. For example, the term $\mathcal{A}_{R, 1}$ corresponds to $r>\theta$ and $s>\theta^{\prime}$ :

$$
\begin{aligned}
\mathcal{A}_{R, 1}= & \frac{1}{4} \int_{[0, t]^{6} \times \mathbb{R}^{6 d}} d r d r^{\prime} d s d s^{\prime} d \theta d \theta^{\prime} d z d z^{\prime} d y d y^{\prime} d w d w^{\prime} \\
& \times \gamma_{0}\left(r-r^{\prime}\right) \gamma_{0}\left(s-s^{\prime}\right) \gamma_{0}\left(\theta-\theta^{\prime}\right) \\
& \times \gamma\left(w-w^{\prime}\right) \gamma\left(y-y^{\prime}\right) \gamma\left(z-z^{\prime}\right) G_{r-\theta}(z-w) G_{s-\theta^{\prime}}\left(y-w^{\prime}\right) \\
& \times \int_{B_{R}^{4}} d x_{4} G_{t-r}\left(x_{1}-z\right) G_{t-s}\left(x_{2}-y\right) G_{t-r^{\prime}}\left(x_{3}-z^{\prime}\right) G_{t-s^{\prime}}\left(x_{4}-y^{\prime}\right) .
\end{aligned}
$$

The term $\mathcal{A}_{R, 2}$ corresponds to $r>\theta$ and $s<\theta^{\prime}$, the term $\mathcal{A}_{R, 3}$ corresponds to $r<\theta$ and $s>\theta^{\prime}$ and the term $\mathcal{A}_{R, 4}$ corresponds to $r<\theta$ and $s<\theta^{\prime}$. In the following, we estimate $\mathcal{A}_{R, j}$ for $j=1,2,3,4$ by a constant times $R^{d}$, which yields (4.31).

To get the bound for $\mathcal{A}_{R, 1}$, it suffices to perform the integration with respect to $d x_{1}, d x_{2}, d x_{4}, d y^{\prime}, d y, d w^{\prime}, d w, d z, d z^{\prime}, d x_{3}$ one by one, by taking into account the following facts:

$$
\sup _{z \in \mathbb{R}^{d}} \int_{B_{R}} G_{t-r}(x-z) d x \leq t \text { and } \sup _{y^{\prime} \in \mathbb{R}^{d}} \int_{\mathbb{R}^{d}} \gamma\left(y-y^{\prime}\right) d y=\|\gamma\|_{L^{1}\left(\mathbb{R}^{d}\right)} \text {. }
$$


To get the bound for $\mathcal{A}_{R, 2}$, it suffices to perform the integration with respect to $d x_{1}, d x_{3}, d z^{\prime}, d z, d x_{2}, d w, d w^{\prime}, d y, d y^{\prime}, d x_{4}$. To get the bound for $\mathcal{A}_{R, 3}$, it suffices to perform the integration with respect to $d x_{4}, d y^{\prime}, d x_{2}, d y, d w^{\prime}, d x_{1}, d w, d z, d z^{\prime}, d x_{3}$ one by one. To get the bound for $\mathcal{A}_{R, 4}$, it suffices to perform the integration with respect to $d x_{1}, d x_{3}, d x_{2}, d z^{\prime}, d z, d w, d w^{\prime}, d y, d y^{\prime}, d x_{4}$ one by one. This completes the proof of (4.29).

In the second part of this subsection, we show the f.d.d. convergence in Theorem 1.4-(1).

Fix an integer $m \geq 1$ and choose $t_{1}, \ldots, t_{m} \in(0, \infty)$. Put $\mathbf{F}_{R}$ $=\left(F_{R}\left(t_{1}\right), \ldots, F_{R}\left(t_{m}\right)\right)$. Then, by the result on limiting covariance structure from Sect. 4.1.1, we have that the covariance matrix of $R^{-d / 2} \mathbf{F}_{R}$, denoted by $\mathcal{C}_{R}$, converges to the matrix $\mathcal{C}=\left(\mathcal{C}_{i j}: 1 \leq i, j \leq m\right)$, with

$$
\mathcal{C}_{i j}=\omega_{d} \sum_{p \geq 1} p ! \int_{\mathbb{R}^{d}}\left\langle\widetilde{f}_{t_{i}, x, p}, \widetilde{f}_{t_{j}, 0, p}\right\rangle_{\mathcal{H} \otimes p} d x
$$

Since $F_{R}(t)=\delta\left(-D L^{-1} F_{R}(t)\right)$, according to [25, Theorem 6.1.2], ${ }^{11}$ for any twice differentiable function $h: \mathbb{R}^{m} \rightarrow \mathbb{R}$ with bounded second partial derivatives,

$$
\begin{aligned}
& \left|\mathbb{E}\left[h\left(R^{-d / 2} \mathbf{F}_{R}\right)-h(\mathbf{Z})\right]\right| \leq\left|\mathbb{E}\left[h\left(R^{-d / 2} \mathbf{F}_{R}\right)-h\left(\mathbf{Z}_{R}\right)\right]\right|+\left|\mathbb{E}\left[h(\mathbf{Z})-h\left(\mathbf{Z}_{R}\right)\right]\right| \\
& \quad \leq \frac{m}{2 R^{d}}\left\|h^{\prime \prime}\right\|_{\infty} \sqrt{\sum_{i, j=1}^{m} \operatorname{Var}\left(\left\langle D F_{R}\left(t_{i}\right),-D L^{-1} F_{R}\left(t_{j}\right)\right\rangle_{\mathcal{H}}\right)}+\left|\mathbb{E}\left[h(\mathbf{Z})-h\left(\mathbf{Z}_{R}\right)\right]\right|,
\end{aligned}
$$

with $\mathbf{Z}_{R} \sim N\left(0, \mathcal{C}_{R}\right), \mathbf{Z} \sim N(0, \mathcal{C})$ and $\left\|h^{\prime \prime}\right\|_{\infty}=\sup \left\{\left|\frac{\partial^{2}}{\partial x_{i} \partial x_{j}} h(x)\right|: x \in \mathbb{R}^{m}, i, j=\right.$ $1, \ldots, m\}$. It is clear that the second term in (4.35) tends to zero as $R \rightarrow \infty$. For the variance term in (4.35), taking advantage of Proposition 1.9 applied to $F=F_{R}\left(t_{i}\right)$ and $G=F_{R}\left(t_{j}\right)$ and using arguments analogous to those employed to derive (4.31), we obtain

$$
\operatorname{Var}\left(\left\langle D F_{R}\left(t_{i}\right),-D L^{-1} F_{R}\left(t_{j}\right)\right\rangle_{\mathcal{H}}\right) \lesssim R^{d}
$$

Thus, the first term in (4.35) is $O\left(R^{-d / 2}\right)$, implying that $\mathbb{E}\left[h\left(R^{-d / 2} \mathbf{F}_{R}\right)-h(\mathbf{Z})\right]$ converges to zero as $R \rightarrow \infty$. This shows the convergence of the finite-dimensional distributions of $\left\{R^{-d / 2} F_{R}(t): t \in \mathbb{R}_{+}\right\}$to those of the centered Gaussian process $\mathcal{G}$, whose covariance structure is given by

$$
\mathbb{E}[\mathcal{G}(t) \mathcal{G}(s)]=\omega_{d} \sum_{p \geq 1} p ! \int_{\mathbb{R}^{d}}\left\langle\widetilde{f}_{t, x, p}, \widetilde{f}_{s, 0, p}\right\rangle_{\mathcal{H}^{\otimes p}} d x \text {, for } s, t \in[0, \infty) .
$$

This concludes the proof of part (1) in Theorem 1.4.

\footnotetext{
11 Note that there is a typo in Theorem 6.1.2 of [25]: In (6.1.3) of [25], one has $d / 2$ instead of $1 / 2$.
} 


\subsubsection{Proofs in parts (2) and (3)}

In part (2), in view of the dominance of the first chaos, we have already obtained in Sect. 4.1.2 that the finite-dimensional distributions of the process $\left\{R^{-d+\frac{\beta}{2}} F_{R}(t)\right.$ : $\left.t \in \mathbb{R}_{+}\right\}$converge to those of a centered Gaussian process $\left\{\mathcal{G}_{\beta}(t)\right\}_{t \in \mathbb{R}_{+}}$, whose covariance structure is given by (1.19). By the same reason, the convergence of the finite-dimensional distributions in part (3) follows from (4.24), (4.25), (4.27) and (4.28).

In this section, we show that:

$$
d_{\mathrm{TV}}\left(F_{R}(t) / \sigma_{R}(t), Z\right) \lesssim \begin{cases}R^{-\beta / 2} & \text { in part (2), } \\ R^{-\frac{1}{2}\left(\beta_{1}+\beta_{2}\right)} & \text { in part (3) case }\left(a^{\prime}\right), \\ R^{-(1+\beta) / 2} & \text { in part (3) case }\left(b^{\prime}\right),\end{cases}
$$

where $Z \sim N(0,1)$. Taking into account (4.30) and the variance estimates in Sects. 4.1.2 and 4.1.3, in order to get (4.36) it suffices to show that, for $j \in\{1,2,3,4\}$ and for $R \geq t$

$$
\mathcal{A}_{R, j} \lesssim \begin{cases}R^{4 d-3 \beta} & \text { in part }(2) \\ R^{8-3\left(\beta_{1}+\beta_{2}\right)} & \text { in case }\left(a^{\prime}\right) \text { of part (3) } \\ R^{5-3 \beta} & \text { in case }\left(b^{\prime}\right) \text { of part (3) }\end{cases}
$$

Since the total-variation distance is always bounded by one, the bound (4.36) still holds for $R<t$ by choosing the implicit constant large enough.

The rest of this section is then devoted to proving (4.37) for $R \geq t$ and for $j \in$ $\{1,2,3,4\}$.

Proof of (4.37) Let us first consider the term $\mathcal{A}_{R, 1}$, which can be expressed as

$$
\mathcal{A}_{R, 1}=\int_{[0, t]^{6}} d r d r^{\prime} d s d s^{\prime} d \theta d \theta^{\prime} \gamma_{0}\left(r-r^{\prime}\right) \gamma_{0}\left(s-s^{\prime}\right) \gamma_{0}\left(\theta-\theta^{\prime}\right) \mathbf{S}_{1, R}
$$

with

$$
\begin{aligned}
\mathbf{S}_{1, R}:= & \int_{\mathbb{R}^{6 d}} d z d z^{\prime} d y d y^{\prime} d w d w^{\prime} \gamma\left(w-w^{\prime}\right) \gamma\left(y-y^{\prime}\right) \gamma\left(z-z^{\prime}\right) \int_{B_{R}^{4}} d \boldsymbol{x}_{4} G_{t-r}\left(x_{1}-z\right) \\
& \times G_{r-\theta}(z-w) G_{t-s}\left(x_{2}-y\right) G_{S-\theta^{\prime}}\left(y-w^{\prime}\right) G_{t-r^{\prime}}\left(x_{3}-z^{\prime}\right) G_{t-s^{\prime}}\left(x_{4}-y^{\prime}\right) .
\end{aligned}
$$

From now on, when $d=2$, we write $\left(w, w^{\prime}, y, y^{\prime}, z, z^{\prime}\right)=\left(w_{1}, w_{2}, w_{1}^{\prime}, w_{2}^{\prime}, y_{1}, y_{2}\right.$, $\left.y_{1}^{\prime}, y_{2}^{\prime}, z_{1}, z_{2}, z_{1}^{\prime}, z_{2}^{\prime}\right)$ and then $d y=d y_{1} d y_{2}$; note also that $x_{1}, \ldots, x_{4}$ denote the dummy variables in $\mathbb{R}^{d}$. By making the following change of variables

$$
\left(z, z^{\prime}, y, y^{\prime}, w, w^{\prime}, x_{1}, x_{2}, x_{3}, x_{4}\right) \rightarrow R\left(z, z^{\prime}, y, y^{\prime}, w, w^{\prime}, x_{1}, x_{2}, x_{3}, x_{4}\right)
$$


and using the scaling property $G_{t}(R z)=R^{1-d} G_{t R^{-1}}(z)$ for $d \in\{1,2\}$, we get

$$
\begin{aligned}
& \mathbf{S}_{1, R}=R^{6+4 d} \int_{[-2,2]^{6 d}} d z d z^{\prime} d y d y^{\prime} d w d w^{\prime} \gamma\left(R w-R w^{\prime}\right) \gamma\left(R y-R y^{\prime}\right) \gamma\left(R z-R z^{\prime}\right) \\
& \quad \int_{B_{1}^{4}} d \boldsymbol{x}_{\mathbf{4}} \times G_{\frac{t-r}{R}}\left(x_{1}-z\right) G_{\frac{r-\theta}{R}}(z-w) G_{\frac{t-s}{R}}\left(x_{2}-y\right) G_{\frac{s-\theta^{\prime}}{R}}\left(y-w^{\prime}\right) \\
& G_{\frac{t-r^{\prime}}{R}}\left(x_{3}-z^{\prime}\right) G_{\frac{t-s^{\prime}}{R}}\left(x_{4}-y^{\prime}\right)
\end{aligned}
$$

Note that we have replaced the integral domain $\mathbb{R}^{6 d}$ by $[-2,2]^{6 d}$ in (4.39) without changing the value of $\mathbf{S}_{1, R}$, because, for example, $x_{1} \in B_{1}$ and $\left|x_{1}-z\right| \leq(t-r) / R$ implies $|z| \leq 1+t R^{-1} \leq 2$ while $|z-w| \leq(r-\theta) / R$ and $\left|x_{1}-z\right| \leq(t-r) / R$ imply $|w| \leq(t-\theta) R^{-1}+1 \leq 2$.

In view of the expression of $\gamma$ in part (2) and part (3), we write, for $z \in \mathbb{R}^{d}$ $\left(z=\left(z_{1}, z_{2}\right) \in \mathbb{R}^{2}\right.$ when $\left.d=2\right)$,

$$
\gamma(R z)= \begin{cases}R^{-\beta} \gamma(z) & \text { in part (2), } \\ R^{-\beta_{1}-\beta_{2} \gamma(z)} & \text { in case }\left(a^{\prime}\right) \text { of part (3), } \\ R^{-\beta} \gamma_{1}\left(R z_{1}\right) \gamma_{2}\left(z_{2}\right) & \text { in case }\left(b^{\prime}\right) \text { of part (3), }\end{cases}
$$

and it is easy to see that

$$
\begin{aligned}
& \sup _{z^{\prime} \in[-2,2]^{d}} \int_{[-2,2]^{d}} \gamma\left(R z-R z^{\prime}\right) d z \\
& \leq \begin{cases}R^{-\beta} \int_{[-4,4]^{d}} \gamma(z) d z<\infty \quad \text { in part }(2), \\
R^{-\beta_{1}-\beta_{2}} \int_{[-4,4]^{d}} \gamma(z) d z<\infty \quad \text { in case }\left(a^{\prime}\right) \text { of part }(3), \\
R^{-\beta-1} \gamma_{1}(\mathbb{R}) \int_{-4}^{4} \gamma_{2}(s) d s<\infty & \text { in case }\left(b^{\prime}\right) \text { of part }(3) .\end{cases}
\end{aligned}
$$

To ease the notation, we just rewrite the above estimates as

$$
\sup _{z^{\prime} \in[-2,2]^{d}} \int_{[-2,2]^{d}} \gamma\left(R z-R z^{\prime}\right) d z \lesssim R^{-\alpha}
$$

with $\alpha=\beta$ in part (2), $\alpha=\beta_{1}+\beta_{2}$ in case $\left(a^{\prime}\right)$ of part (3), and $\alpha=1+\beta$ in case $\left(b^{\prime}\right)$ of part (3).

To estimate $\mathcal{A}_{R, 1}$, we can use (4.40) to perform integration with respect to $d x_{1}, d x_{2}, d x_{4}, d y^{\prime}, d y, d w^{\prime}, d w, d z, d z^{\prime}, d x_{3}$ successively. More precisely, perform- 
ing the integration with respect to $d x_{1}, d x_{2}, d x_{4}$ and using the fact

$$
\sup _{\left(s, z^{\prime}\right) \in[0, t] \times \mathbb{R}^{d}} \int_{\mathbb{R}^{d}} G_{S / R}\left(z-z^{\prime}\right) d z=t / R
$$

gives us

$$
\begin{aligned}
\mathbf{S}_{1, R} \leq & R^{3+4 d} t^{3} \int_{[-2,2]^{6 d}} d z d z^{\prime} d y d y^{\prime} d w d w^{\prime} \gamma\left(R w-R w^{\prime}\right) \\
& \gamma\left(R y-R y^{\prime}\right) \gamma\left(R z-R z^{\prime}\right) \int_{B_{1}} d x_{3} \\
& \quad \times G_{\frac{r-\theta}{R}}(z-w) G_{\frac{s-\theta^{\prime}}{R}}\left(y-w^{\prime}\right) G_{\frac{t-r^{\prime}}{R}}\left(x_{3}-z^{\prime}\right) \\
\lesssim & R^{3+4 d} R^{-\alpha} \int_{[-2,2]^{5 d}} d z d z^{\prime} d y d w d w^{\prime} \gamma\left(R w-R w^{\prime}\right) \gamma\left(R z-R z^{\prime}\right) \int_{B_{1}} d x_{3} \\
& \quad \times G_{\frac{r-\theta}{R}}(z-w) G_{\frac{s-\theta^{\prime}}{R}}\left(y-w^{\prime}\right) G_{\frac{t-r^{\prime}}{R}}\left(x_{3}-z^{\prime}\right) \\
& \quad \text { by integrating out } d y^{\prime} \text { and using }(4.40) \\
\lesssim & R^{2+4 d-\alpha} \int_{[-2,2]^{4 d}} d z d z^{\prime} d w d w^{\prime} \gamma\left(R w-R w^{\prime}\right) \gamma\left(R z-R z^{\prime}\right) \int_{B_{1}} d x_{3} \\
& \quad \times G_{\frac{r-\theta}{R}}(z-w) G_{\frac{t-r^{\prime}}{R}}\left(x_{3}-z^{\prime}\right) \text { by integrating out } d y \text { and using }(4.41) \\
\lesssim & R^{2+4 d-2 \alpha} \int_{[-2,2]^{3 d}} d z d z^{\prime} d w \gamma\left(R z-R z^{\prime}\right) \int_{B_{1}} d x_{3} G_{\frac{r-\theta}{R}}(z-w) G_{\frac{t-r^{\prime}}{R}}\left(x_{3}-z^{\prime}\right)
\end{aligned}
$$

by integrating out $d w^{\prime}$ and using (4.40); then, using (4.41) to integrate out $d w$

$$
\lesssim R^{1+4 d-2 \alpha} \int_{[-2,2]^{2 d}} d z d z^{\prime} \gamma\left(R z-R z^{\prime}\right) \int_{B_{1}} d x_{3} G_{\frac{t-r^{\prime}}{R}}\left(x_{3}-z^{\prime}\right) \lesssim R^{4 d-3 \alpha}
$$

where the last inequality is obtained by integrating out $d z, d z^{\prime}, d x_{3}$ one by one and using (4.40) and (4.41). The bound

$$
\mathbf{S}_{1, R} \lesssim R^{4 d-3 \alpha}= \begin{cases}R^{4 d-3 \beta} & \text { in part (2), } \\ R^{8-3 \beta_{1}-3 \beta_{2}} & \text { in cae }\left(a^{\prime}\right) \text { of part (3) } \\ R^{5-3 \beta} & \text { in cae }\left(b^{\prime}\right) \text { of part (3) }\end{cases}
$$

is uniform over $\left(r, r^{\prime}, s, s^{\prime}, \theta, \theta^{\prime}\right) \in[0, t]^{6}$, and hence we obtain (4.37) for $j=1$. For the other terms $\mathcal{A}_{R, 2}, \mathcal{A}_{R, 3}$ and $\mathcal{A}_{R, 4}$, the arguments are the same: We first go through the same change of variables (4.38) to obtain terms $\mathbf{S}_{j, R}$ similar to $\mathbf{S}_{1, R}$ in (4.39), and then use the facts (4.40) and (4.41) to perform one-by-one integration with respect to 
the variables

$$
\begin{cases}d x_{1}, d x_{3}, d z^{\prime}, d z, d x_{2}, d w, d w^{\prime}, d y, d y^{\prime}, d x_{4} & \text { for estimating } \mathcal{A}_{R, 2} \\ d x_{4}, d y^{\prime}, d x_{2}, d y, d w^{\prime}, d x_{1}, d w, d z, d z^{\prime}, d x_{3} & \text { for estimating } \mathcal{A}_{R, 3} \\ d x_{1}, d x_{3}, d x_{2}, d z^{\prime}, d z, d w, d w^{\prime}, d y, d y^{\prime}, d x_{4} & \text { for estimating } \mathcal{A}_{R, 4}\end{cases}
$$

This concludes the proof of (4.37) and hence completes the proof of (4.36).

\subsection{Tightness}

This section is devoted to establishing the tightness in Theorem 1.4. This, together with the results in Sects. 4.1 and 4.2 will conclude the proof of Theorem 1.4. To get the tightness, we appeal to the criterion of Kolmogorov-Chentsov (see e.g. [17, Corollary 16.9]). Put

$$
\sigma_{R}= \begin{cases}R^{d / 2} & \text { in part (1) of Theorem } 1.4 \\ R^{d-\frac{\beta}{2}} & \text { in part (2) of Theorem } 1.4 \\ R^{2-\frac{1}{2}\left(\beta_{1}+\beta_{2}\right)} & \text { in part . }(3)-\left(a^{\prime}\right) \text { of Theorem } 1.4 \\ R^{(3-\beta) / 2} & \text { in part }(3)-\left(b^{\prime}\right) \text { of Theorem } 1.4\end{cases}
$$

and we will show, for any fixed $T>0$, that the following inequality holds for any integer $k \geq 2$ and any $0<s<t \leq T \leq R$ :

$$
\left\|F_{R}(t)-F_{R}(s)\right\|_{k} \lesssim(t-s) \sigma_{R}
$$

where the implicit constant does not depend on $R, s$ or $t$. This moment estimate (4.43) ensures the tightness of $\left\{\sigma_{R}^{-1} F_{R}(t): t \in[0, T]\right\}$ for any fixed $T>0$ and, therefore, the desired tightness on $\mathbb{R}_{+}$holds.

To show the above moment estimate (4.43) for the increment $F_{R}(t)-F_{R}(s)$, we begin with the chaos expansion

$$
F_{R}(t)-F_{R}(s)=\sum_{n \geq 1} I_{n}\left(\int_{B_{R}} d x\left[f_{t, x, n}-f_{s, x, n}\right]\right)=\sum_{n \geq 1} I_{n}\left(g_{n, R}\right),
$$

where $s, t$ are fixed, so we leave them out of the subscript of the kernel $g_{n, R}$ and

$$
g_{n, R}\left(\boldsymbol{s}_{\boldsymbol{n}}, \boldsymbol{y}_{\boldsymbol{n}}\right)=\left[\varphi_{t, R}\left(s_{1}, y_{1}\right)-\varphi_{s, R}\left(s_{1}, y_{1}\right)\right] \prod_{j=1}^{n-1} G_{s_{j}-s_{j+1}}\left(y_{j}-y_{j+1}\right)
$$

with $\prod_{j=1}^{0}=1$ and $\varphi_{t, R}(r, y):=\int_{B_{R}} G_{t-r}(x-y) d x$. The rest of this section is then devoted to proving (4.43). 
Proof of (4.43) By the triangle inequality and using the moment estimate (2.15), we get, for any $k \in[2, \infty)$,

$$
\left\|F_{R}(t)-F_{R}(s)\right\|_{k} \leq \sum_{n \geq 1}(k-1)^{n / 2}\left\|I_{n}\left(g_{n, R}\right)\right\|_{2} .
$$

Note that the kernel $g_{n, R}=0$ outside $[0, t]^{n} \times \mathbb{R}^{d n}$. Then, using (2.8) and (2.13), we can write

$$
\left\|F_{R}(t)-F_{R}(s)\right\|_{k} \leq \sum_{n \geq 1}\left[\Gamma_{t}(k-1)\right]^{n / 2}\left(n !\left\|\widetilde{g}_{n, R}\right\|_{\mathcal{H}_{0}^{\otimes n}}^{2 n}\right)^{1 / 2}
$$

where $\widetilde{g}_{n, R}$ is the canonical symmetrization of $g_{n, R}$ :

$$
\begin{aligned}
\widetilde{g}_{n, R}\left(\boldsymbol{s}_{\boldsymbol{n}}, \boldsymbol{y}_{\boldsymbol{n}}\right)= & \frac{1}{n !} \sum_{\sigma \in \mathfrak{S}_{n}}\left[\varphi_{t, R}\left(s_{\sigma(1)}, y_{\sigma(1)}\right)-\varphi_{s, R}\left(s_{\sigma(1)}, y_{\sigma(1)}\right)\right] \\
& \times \prod_{j=1}^{n-1} G_{S_{\sigma(j)}-s_{\sigma(j+1)}}\left(y_{\sigma(j)}-y_{\sigma(j+1)}\right) .
\end{aligned}
$$

With the convention (1.6) in mind, we can write

$$
\begin{aligned}
& n !\left\|\widetilde{g}_{n, R}\right\|_{\mathcal{H}_{0}^{\otimes n}}^{2}=\int_{t>s_{1}>\cdots>s_{n}>0} d \boldsymbol{s}_{\boldsymbol{n}} \int_{\mathbb{R}^{2 n d}}\left[\varphi_{t, R}\left(s_{1}, y_{1}\right)-\varphi_{s, R}\left(s_{1}, y_{1}\right)\right] \\
& \times\left(\prod_{j=1}^{n-1} G_{s_{j}-s_{j+1}}\left(y_{j}-y_{j+1}\right)\right) \times\left[\varphi_{t, R}\left(s_{1}, y_{1}^{\prime}\right)-\varphi_{s, R}\left(s_{1}, y_{1}^{\prime}\right)\right] \\
& \quad \times\left(\prod_{j=1}^{n-1} G_{s_{j}-s_{j+1}}\left(y_{j}^{\prime}-y_{j+1}^{\prime}\right)\right) \prod_{j=1}^{n} \gamma\left(y_{j}-y_{j}^{\prime}\right) d y_{j} d y_{j}^{\prime} .
\end{aligned}
$$

Then, using Fourier transform, we can rewrite $n !\left\|\widetilde{g}_{n, R}\right\|_{\mathcal{H}_{0}^{\otimes n}}^{2}$ as follows:

$$
\begin{aligned}
& n !\left\|\widetilde{g}_{n, R}\right\|_{\mathcal{H}_{0}^{\otimes n}}^{2}=\int_{t>s_{1}>\cdots>s_{n}>0} d \boldsymbol{s}_{\boldsymbol{n}} \int_{\mathbb{R}^{n d}} \mu\left(d \boldsymbol{\xi}_{\boldsymbol{p}}\right)\left|\mathcal{F} \mathbf{1}_{B_{R}}\right|^{2}\left(\xi_{1}+\cdots+\xi_{p}\right) \\
& \quad \times\left|\widehat{G}_{t-t_{1}}\left(\xi_{1}+\cdots+\xi_{p}\right)-\widehat{G}_{s-t_{1}}\left(\xi_{1}+\cdots+\xi_{p}\right)\right|^{2} \\
& \quad \times \prod_{j=1}^{n-1}\left|\widehat{G}_{s_{j}-s_{j+1}}\right|^{2}\left(\xi_{j+1}+\cdots+\xi_{p}\right)
\end{aligned}
$$


Recall the expression (2.29) $\widehat{G}_{t}(\xi)=\frac{\sin (t|\xi|)}{|\xi|}$ and note that it is a 1-Lipschitz function in the variable $t$, uniformly over $\xi \in \mathbb{R}^{d}$. Then

$$
\left|\widehat{G}_{t-t_{1}}\left(\xi_{1}+\cdots+\xi_{p}\right)-\widehat{G}_{s-t_{1}}\left(\xi_{1}+\cdots+\xi_{p}\right)\right|^{2} \leq(t-s)^{2} .
$$

Therefore, plugging this inequality into (4.45) and then applying Lemma 2.6 yields

$$
\begin{aligned}
n !\left\|\widetilde{g}_{n, R}\right\|_{\mathcal{H}_{0}^{\otimes n}}^{\otimes n} \leq & (t-s)^{2} \int_{t>s_{1}>\cdots>s_{n}>0} d \boldsymbol{s}_{\boldsymbol{n}}\left(\int_{\mathbb{R}^{d}} \mu(d \xi)\left|\mathcal{F} \mathbf{1}_{B_{R}}\right|^{2}(\xi)\right) \\
& \times \prod_{j=1}^{n-1} \int_{\mathbb{R}^{d}} \mu\left(d \xi \xi_{j}\right)\left|\widehat{G}_{s_{j}-s_{j+1}}\right|^{2}\left(\xi_{j}\right) \\
\leq & (t-s)^{2} \frac{t^{n}}{n !}\left(2\left(t^{2} \vee 1\right) \int_{\mathbb{R}^{d}} \frac{\mu(d \xi)}{1+|\xi|^{2}}\right)^{n-1} \int_{\mathbb{R}^{d}} \mu(d \xi)\left|\mathcal{F} \mathbf{1}_{B_{R}}\right|^{2}(\xi),
\end{aligned}
$$

which is finite since $\mathbf{1}_{B_{R}} \in \mathcal{P}_{0}$. Using Fourier transform, we can write

$$
\int_{\mathbb{R}^{d}} \mu(d \xi)\left|\mathcal{F} \mathbf{1}_{B_{R}}\right|^{2}(\xi)=\int_{\mathbb{R}^{2 d}} \mathbf{1}_{B_{R}}(x) \mathbf{1}_{B_{R}}(y) \gamma(x-y) d x d y .
$$

Now let us consider the cases in (4.42).

In part (1) where $\gamma \in L^{1}\left(\mathbb{R}^{d}\right)$,

$$
\int_{\mathbb{R}^{2 d}} \mathbf{1}_{B_{R}}(x) \mathbf{1}_{B_{R}}(y) \gamma(x-y) d x d y \leq \gamma\left(\mathbb{R}^{d}\right) \omega_{d} R^{d} \lesssim \sigma_{R}^{2} .
$$

In the other cases, we can make the change of variables $(x, y) \rightarrow R(x, y)$ to obtain

$$
\begin{aligned}
\int_{\mathbb{R}^{2 d}} \mathbf{1}_{B_{R}}(x) \mathbf{1}_{B_{R}}(y) \gamma(x-y) d x d y & =R^{2 d} \int_{\mathbb{R}^{2 d}} \mathbf{1}_{B_{1}}(x) \mathbf{1}_{B_{1}}(y) \gamma(R x-R y) d x d y \\
& \lesssim R^{2 d-\alpha}=\sigma_{R}^{2}
\end{aligned}
$$

using (4.40) with $\alpha=\beta$ in part (2), $\alpha=\beta_{1}+\beta_{2}$ in case $\left(a^{\prime}\right)$, and $\alpha=1+\beta$ in case $\left(b^{\prime}\right)$.

As a consequence, we get

$$
n !\left\|\widetilde{g}_{n, R}\right\|_{\mathcal{H}_{0}^{\otimes n}}^{2} \leq \frac{C^{n}}{n !} \sigma_{R}^{2}(t-s)^{2},
$$

and therefore,

$$
\left\|F_{R}(t)-F_{R}(s)\right\|_{k} \leq|t-s| \sigma_{R} \sum_{n \geq 1}\left[C \Gamma_{t}(k-1)\right]^{n / 2} \frac{1}{\sqrt{n !}},
$$

which leads to (4.43). 


\section{Proof of Theorem 1.10}

We argue as in the proof of Theorem 1.2 of [2]. As we explained in the introduction, it suffices to show that for each $m \geq 1$,

$$
\|D u(t, x)\|_{\mathcal{H}}>0 \text { a.s. on } \Omega_{m}
$$

where $\Omega_{m}=\{|u(t, x)| \geq 1 / m\}$.

We claim that, almost surely, the function $(s, y) \mapsto D_{s, y} u(t, x)$ satisfies the assumptions of Lemma A.1. Indeed, for $d=2$, by Minkowski's inequality and the estimate (1.11), we have

$$
\begin{aligned}
\mathbb{E}\left(\int_{0}^{t} d s\left(\int_{\mathbb{R}^{2}}\left|D_{s, y} u(t, x)\right|^{2 q} d y\right)^{1 / q}\right) & \leq \int_{0}^{t} d s\left(\int_{\mathbb{R}^{2}}\left|\mathbb{E}\left[\left|D_{s, y} u(t, x)\right|^{2}\right]\right|^{q} d y\right)^{1 / q} \\
& \leq C \int_{0}^{t} d s\left(\int_{\mathbb{R}^{2}} G_{t-s}^{2 q}(x-y) d y\right)^{1 / q}<\infty .
\end{aligned}
$$

For $d=1$, again by the estimate (1.11),

$$
\mathbb{E}\left(\int_{0}^{t} d s\left(\int_{\mathbb{R}}\left|D_{s, y} u(t, x)\right|^{2} d y\right)\right) \leq C \int_{0}^{t} d s \int_{\mathbb{R}} G_{t-s}^{2}(x-y) d y<\infty .
$$

Moreover, $(s, y) \mapsto D_{s, y} u(t, x)$ has compact support on $[0, t] \times B_{M}$ for some $M>0$. As a consequence, by Lemma A.1, it suffices to prove that

$$
\begin{aligned}
& \int_{0}^{t}\left\|D_{r, \bullet} u(t, x)\right\|_{0}^{2} d r \\
& \quad=\int_{0}^{t} \int_{\mathbb{R}^{2 d}} D_{r, z} u(t, x) D_{r, z^{\prime}} u(t, x) \gamma\left(z-z^{\prime}\right) d z d z^{\prime} d r>0 \text { a.s. on } \Omega_{m} .
\end{aligned}
$$

As in the proof of Lemma 5.1 of [2], Corollaries 3.3 and 3.4 allow us to infer that the $\mathcal{H} \otimes \mathcal{P}_{0}$-valued process $K^{(r)}$ defined by

$$
K^{(r)}(s, y, z)=G_{t-s}(x-y) D_{r, z} u(s, y)
$$

belongs to the space $\mathbb{D}^{1,2}\left(\mathcal{H} \otimes \mathcal{P}_{0}\right)$. This is because, using Corollary 3.3 , we can write

$$
\begin{aligned}
\mathbb{E}( & \left.\left\|K^{(r)}\right\|_{\mathcal{H} \otimes \mathcal{P}_{0}}^{2}\right) \\
= & \int_{[r, t]^{2}} \int_{\mathbb{R}^{2 d}} G_{t-s}(x-y) G_{t-s^{\prime}}\left(x-y^{\prime}\right) \mathbb{E}\left(\left\langle D_{r, \bullet} u(s, y), D_{r, \bullet} u\left(s^{\prime}, y^{\prime}\right)\right\rangle_{0}\right) \\
& \times \gamma_{0}\left(s-s^{\prime}\right) \gamma\left(y-y^{\prime}\right) d y d y^{\prime} d s d s^{\prime} \\
& \leq C \int_{[r, t]^{2}} \int_{\mathbb{R}^{2 d}} G_{t-s}(x-y) G_{t-s^{\prime}}\left(x-y^{\prime}\right)
\end{aligned}
$$




$$
\gamma_{0}\left(s-s^{\prime}\right) \gamma\left(y-y^{\prime}\right) d y d y^{\prime} d s d s^{\prime}<\infty
$$

and in the same way, using Corollary 3.4 we can show that $\mathbb{E}\left(\left\|D K^{(r)}\right\|_{\mathcal{H} \otimes \mathcal{H} \otimes \mathcal{P}_{0}}^{2}\right)<$ $\infty$. Therefore, the process $K^{(r)}$ belongs to the domain of the $\mathcal{P}_{0}$-valued Skorokhod integral, denoted by $\bar{\delta}$. Then, using the same arguments as in the proof of Proposition 5.2 of [2], replacing $L^{2}(\mathbb{R})$ by $\mathcal{P}_{0}$, we can show that for any $r \in[0, t]$, the following equation holds in $L^{2}\left(\Omega ; \mathcal{P}_{0}\right)$ :

$$
D_{r, \bullet} u(t, x)=G_{t-r}(x-\bullet) u(r, \bullet)+\int_{r}^{t} \int_{\mathbb{R}^{d}} G_{t-s}(x-y) D_{r, \bullet} u(s, y) W(\bar{\delta} s, \bar{\delta} y) .
$$

Let $\delta \in(0, t \wedge 1)$ be arbitrary. Due to relation (5.2) we have, almost surely,

$$
\begin{aligned}
\int_{0}^{t}\left\|D_{r, \bullet} u(t, x)\right\|_{0}^{2} d r & \geq \int_{t-\delta}^{t}\left\|D_{r, \bullet} u(t, x)\right\|_{0}^{2} d r \\
& \geq \frac{1}{2} \int_{t-\delta}^{t}\left\|G_{t-r}(x-\bullet) u(r, \bullet)\right\|_{0}^{2} d r-I(\delta),
\end{aligned}
$$

where

$$
\begin{aligned}
I(\delta) & =\int_{t-\delta}^{t}\left\|\int_{r}^{t} \int_{\mathbb{R}^{d}} G_{t-s}(x-y) D_{r, \bullet} u(s, y) W(\bar{\delta} s, \bar{\delta} y)\right\|_{0}^{2} d r \\
& =\int_{t-\delta}^{t}\left\|\int_{t-\delta}^{t} \int_{\mathbb{R}^{d}} G_{t-s}(x-y) D_{r, \bullet} u(s, y) W(\bar{\delta} s, \bar{\delta} y)\right\|_{0}^{2} d r .
\end{aligned}
$$

On the event $\Omega_{m}=\{|u(t, x)| \geq 1 / m\}$, we have

$$
\begin{aligned}
& \int_{t-\delta}^{t}\left\|G_{t-r}(x-\bullet) u(r, \bullet)\right\|_{0}^{2} d r \\
& \quad=\int_{t-\delta}^{t} \int_{\mathbb{R}^{2 d}} G_{t-r}(x-z) G_{t-r}\left(x-z^{\prime}\right) u(r, z) u\left(r, z^{\prime}\right) \gamma\left(z-z^{\prime}\right) d z d z^{\prime} d r \\
& \quad=\int_{t-\delta}^{t} \int_{\mathbb{R}^{2 d}} G_{t-r}(x-z) G_{t-r}\left(x-z^{\prime}\right) u(t, x)^{2} \gamma\left(z-z^{\prime}\right) d z d z^{\prime} d r \\
& \quad-\int_{t-\delta}^{t} \int_{\mathbb{R}^{2 d}} G_{t-r}(x-z) G_{t-r}\left(x-z^{\prime}\right)\left[u(t, x)^{2}-u(r, z) u\left(r, z^{\prime}\right)\right] \gamma\left(z-z^{\prime}\right) d z d z^{\prime} d r \\
& \quad \geq \frac{1}{m^{2}} \psi_{0}(\delta)-J(\delta),
\end{aligned}
$$

where

$$
\psi_{0}(\delta):=\int_{t-\delta}^{t} \int_{\mathbb{R}^{2 d}} G_{t-r}(x-z) G_{t-r}\left(x-z^{\prime}\right) \gamma\left(z-z^{\prime}\right) d z d z^{\prime} d r
$$




$$
=\int_{0}^{\delta} \int_{\mathbb{R}^{2 d}} G_{r}(z) G_{r}\left(z^{\prime}\right) \gamma\left(z-z^{\prime}\right) d z d z^{\prime} d r
$$

and

$$
\begin{gathered}
J(\delta):=\int_{t-\delta}^{t} \int_{\mathbb{R}^{2 d}} G_{t-r}(x-z) G_{t-r}\left(x-z^{\prime}\right) \gamma\left(z-z^{\prime}\right) \\
\left(u(t, x)^{2}-u(r, z) u\left(r, z^{\prime}\right)\right) d z d z^{\prime} d r .
\end{gathered}
$$

Coming back to (5.3), we can write

$$
\int_{0}^{t}\left\|D_{r, \bullet} u(t, x)\right\|_{0}^{2} d r \geq \frac{1}{2 m^{2}} \psi_{0}(\delta)-\frac{1}{2} J(\delta)-I(\delta) \text { on } \Omega_{m} .
$$

We now give upper bounds for the first moments of $J(\delta)$ and $I(\delta)$. We will use the following facts, which were proved in [3]:

$$
\begin{aligned}
C_{t}^{*} & :=\sup _{(s, y) \in[0, t] \times \mathbb{R}^{d}}\|u(s, y)\|_{2}<\infty \quad \text { (see also (3.16) in Remark 3.1) } \\
g_{t, x}(\delta) & :=\sup _{|t-s|<\delta|x-y|<\delta} \sup _{|x|<(t, x)-u(s, y) \|_{2} \rightarrow 0 \quad \text { as } \delta \rightarrow 0 .} \| u(t)
\end{aligned}
$$

We first treat $J(\delta)$. By Cauchy-Schwarz inequality, for any $r \in[0, t]$ and $z, z^{\prime} \in \mathbb{R}^{2}$,

$$
\begin{aligned}
\mathbb{E}\left[\left|u(t, x)^{2}-u(r, z) u\left(r, z^{\prime}\right)\right|\right] \leq & \|u(t, x)\|_{2}\|u(t, x)-u(r, z)\|_{2} \\
& +\|u(r, z)\|_{2}\left\|u(t, x)-u\left(r, z^{\prime}\right)\right\|_{2} \\
& \leq C_{t}^{*}\left(\|u(t, x)-u(r, z)\|_{2}+\left\|u(t, x)-u\left(r, z^{\prime}\right)\right\|_{2}\right) .
\end{aligned}
$$

Since $G_{t-r}(x-z)$ contains the indicator of the set $\{|x-z|<t-r\}$, we obtain:

$$
\begin{aligned}
\mathbb{E}(|J(\delta)|) \leq & 2 C_{t}^{*} \int_{t-\delta}^{t} \int_{\mathbb{R}^{2 d}} G_{t-r}(x-z) G_{t-r}\left(x-z^{\prime}\right) \gamma\left(z-z^{\prime}\right) \| u(t, x) \\
& -u(r, z) \|_{2} d z d z^{\prime} d r \\
\leq & 2 C_{t}^{*} \int_{t-\delta}^{t} \int_{\mathbb{R}^{2 d}} G_{t-r}(x-z) G_{t-r}\left(x-z^{\prime}\right) \gamma\left(z-z^{\prime}\right) \\
& \sup _{\substack{t-\delta<s<t \\
|x-y|<\delta}}\|u(t, x)-u(s, y)\|_{2} d z d z^{\prime} d r .
\end{aligned}
$$

It follows that

$$
\mathbb{E}(|J(\delta)|) \leq 2 C_{t}^{*} g_{t, x}(\delta) \psi_{0}(\delta) .
$$


Next, we treat $I(\delta)$. Applying Proposition 6.2 of [1] to the $\mathcal{P}_{0}$-valued process

$$
U(s, y)=\mathbf{1}_{[t-\delta, t]}(s) G_{t-s}(x-y) D_{r, \bullet} u(s, y)
$$

we obtain

$$
\mathbb{E}\left(\|\bar{\delta}(U)\|_{0}^{2}\right) \leq \mathbb{E}\left(\|U\|_{\mathcal{H} \otimes \mathcal{P}_{0}}^{2}\right)+\mathbb{E}\left(\|D U\|_{\mathcal{H} \otimes \mathcal{H} \otimes \mathcal{P}_{0}}^{2}\right)
$$

We have,

$$
\begin{aligned}
\mathbb{E}\left(\|U\|_{\mathcal{H} \otimes \mathcal{P}_{0}}^{2}\right)= & \mathbb{E}\left(\int_{[t-\delta, t]^{2}} \int_{\mathbb{R}^{2 d}} G_{t-s}(x-y) G_{t-s^{\prime}}\left(x-y^{\prime}\right) \gamma_{0}\left(s-s^{\prime}\right) \gamma\left(y-y^{\prime}\right)\right. \\
& \left.\times\left\langle D_{r, \bullet} u(s, y), D_{r, \bullet} u\left(s^{\prime}, y^{\prime}\right)\right\rangle_{0} d y d y^{\prime} d s d s^{\prime}\right)
\end{aligned}
$$

and

$$
\begin{aligned}
\mathbb{E}\left(\|D U\|_{\mathcal{H} \otimes \mathcal{H} \otimes \mathcal{P}_{0}}^{2}\right) & \\
= & \mathbb{E}\left(\int_{[t-\delta, t]^{2}} \int_{[0, r]^{2}} \int_{\mathbb{R}^{4 d}} G_{t-s}(x-y) G_{t-s^{\prime}}\left(x-y^{\prime}\right) \gamma_{0}\left(s-s^{\prime}\right) \gamma\left(y-y^{\prime}\right)\right. \\
& \times\left\langle D_{(\theta, w),(r, \bullet)}^{2} u(s, y), D_{\left(\theta^{\prime}, w^{\prime}\right),(r, \bullet)} u\left(s^{\prime}, y^{\prime}\right)\right\rangle_{0} \gamma_{0}\left(\theta-\theta^{\prime}\right) \\
& \left.\times \gamma\left(w-w^{\prime}\right) d w d w^{\prime} d y d y^{\prime} d \theta d \theta^{\prime} d s d s^{\prime}\right) \\
= & \mathbb{E}\left(\int_{[t-\delta, t]^{2}} \int_{\mathbb{R}^{2 d}} G_{t-s}(x-y) G_{t-s^{\prime}}\left(x-y^{\prime}\right) \gamma_{0}\left(s-s^{\prime}\right) \gamma\left(y-y^{\prime}\right)\right. \\
& \left.\times\left\langle D D_{r, \bullet} u(s, y), D D_{r, \bullet} u\left(s^{\prime}, y^{\prime}\right)\right\rangle_{\mathcal{H} \otimes \mathcal{P}_{0}} d y d y^{\prime} d s d s^{\prime}\right) .
\end{aligned}
$$

Hence, $\mathbb{E}(I(\delta)) \leq I_{1}(\delta)+I_{2}(\delta)$, where

$$
\begin{aligned}
I_{1}(\delta):= & \mathbb{E}\left(\int_{[t-\delta, t]^{3}} \int_{\mathbb{R}^{2 d}} G_{t-s}(x-y) G_{t-s^{\prime}}\left(x-y^{\prime}\right) \gamma_{0}\left(s-s^{\prime}\right) \gamma\left(y-y^{\prime}\right)\right. \\
& \left.\times\left\langle D_{r, \bullet} u(s, y), D_{r, \bullet} u\left(s^{\prime}, y^{\prime}\right)\right\rangle_{0} d y d y^{\prime} d s d s^{\prime} d r\right)
\end{aligned}
$$

and

$$
\begin{aligned}
I_{2}(\delta):= & \mathbb{E}\left(\int_{[t-\delta, t]^{3}} \int_{\mathbb{R}^{2 d}} G_{t-s}(x-y) G_{t-s^{\prime}}\left(x-y^{\prime}\right) \gamma_{0}\left(s-s^{\prime}\right) \gamma\left(y-y^{\prime}\right)\right. \\
& \left.\times\left\langle D D_{r, \bullet} u(s, y), D D_{r, \bullet} u\left(s^{\prime}, y^{\prime}\right)\right\rangle_{\mathcal{H} \otimes \mathcal{P}_{0}} d y d y^{\prime} d s d s^{\prime} d r\right) .
\end{aligned}
$$


Using Cauchy-Schwarz inequality and Corollaries 3.3 and 3.4, we obtain:

$$
\begin{aligned}
& \mathbb{E}\left(\left|\left\langle D_{r, \bullet} u(s, y), D_{r, \bullet} u\left(s^{\prime}, y^{\prime}\right)\right\rangle_{0}\right|\right) \leq C_{t} \text { and } \\
& \mathbb{E}\left(\left|\left\langle D D_{r, \bullet} u(s, y), D D_{r, \bullet} u\left(s^{\prime}, y^{\prime}\right)\right\rangle_{\mathcal{H} \otimes \mathcal{P}_{0}}\right|\right) \leq C_{t}^{\prime \prime} .
\end{aligned}
$$

Hence,

$$
\mathbb{E}[I(\delta)] \leq\left(C_{t}+C_{t}^{\prime \prime}\right) \delta \phi(\delta),
$$

where

$$
\begin{aligned}
\phi(\delta) & :=\int_{[t-\delta, t]^{2}} \int_{\mathbb{R}^{2 d}} G_{t-s}(x-y) G_{t-s^{\prime}}\left(x-y^{\prime}\right) \gamma_{0}\left(s-s^{\prime}\right) \gamma\left(y-y^{\prime}\right) d y d y^{\prime} d s d s^{\prime} \\
& =\int_{[0, \delta]^{2}} \int_{\mathbb{R}^{2 d}} G_{s}(y) G_{s^{\prime}}\left(y^{\prime}\right) \gamma_{0}\left(s-s^{\prime}\right) \gamma\left(y-y^{\prime}\right) d y d y^{\prime} d s d s^{\prime}
\end{aligned}
$$

Using (5.4), (5.5) and (5.6), we conclude the proof as follows. For any $n \geq 1$,

$$
\begin{aligned}
& \mathbb{P}\left(\left\{\int_{0}^{t}\left\|D_{r, \bullet} u(t, x)\right\|_{0}^{2} d r<\frac{1}{n}\right\} \cap \Omega_{m}\right) \leq \mathbb{P}\left(I(\delta)+\frac{1}{2} J(\delta)>\frac{1}{2 m^{2}} \psi_{0}(\delta)-\frac{1}{n}\right) \\
& \leq\left(\frac{1}{2 m^{2}} \psi_{0}(\delta)-\frac{1}{n}\right)^{-1}\left(\mathbb{E}[I(\delta)]+\frac{1}{2} \mathbb{E}[|J(\delta)|]\right) \\
& \leq \frac{\left(C_{t}+C_{t}^{\prime \prime}\right) \delta \phi(\delta)+C_{t}^{*} g_{t, x}(\delta) \psi_{0}(\delta)}{\frac{1}{2 m^{2}} \psi_{0}(\delta)-\frac{1}{n}} .
\end{aligned}
$$

Letting $n \rightarrow \infty$, we obtain:

$$
\mathbb{P}\left(\left\{\int_{0}^{t}\left\|D_{r, \bullet} u(t, x)\right\|_{0}^{2} d r=0\right\} \cap \Omega_{m}\right) \leq 2 m^{2}\left(\left(C_{t}+C_{t}^{\prime \prime}\right) \delta \frac{\phi(\delta)}{\psi_{0}(\delta)}+C_{t}^{*} g_{t, x}(\delta)\right) .
$$

Note that using Fourier transform and the expression (2.29), we can rewrite (5.7) as

$$
\begin{aligned}
\phi(\delta) & =\int_{[0, \delta]^{2}} \int_{\mathbb{R}^{d}} \widehat{G}_{S}(\xi) \widehat{G}_{s^{\prime}}(\xi) \gamma_{0}\left(s-s^{\prime}\right) \mu(d \xi) d s d s^{\prime} \\
& \leq \int_{[0, \delta]^{2}} \int_{\mathbb{R}^{d}} \frac{1}{2}\left[\widehat{G}_{S}(\xi)^{2}+\widehat{G}_{s^{\prime}}(\xi)^{2}\right] \gamma_{0}\left(s-s^{\prime}\right) \mu(d \xi) d s d s^{\prime} \\
& \leq \Gamma_{\delta} \int_{[0, \delta]} \int_{\mathbb{R}^{d}} \widehat{G}_{S}(\xi)^{2} \mu(d \xi) d s
\end{aligned}
$$

where $\Gamma_{\delta}=2 \int_{0}^{\delta} \gamma_{0}(s) d s$. That is, we have $\phi(\delta) \leq \Gamma_{\delta} \psi_{0}(\delta)$. Finally taking $\delta \rightarrow 0$ proves (5.1), since $g_{t, x}(\delta) \rightarrow 0$ and $\delta \frac{\phi(\delta)}{\psi_{0}(\delta)} \leq \delta \Gamma_{\delta} \rightarrow 0$ as $\delta \rightarrow 0$. 
Acknowledgements The authors would like to thank Wangjun Yuan for carefully proofreading the manuscript and providing a list of typos.

Open Access This article is licensed under a Creative Commons Attribution 4.0 International License, which permits use, sharing, adaptation, distribution and reproduction in any medium or format, as long as you give appropriate credit to the original author(s) and the source, provide a link to the Creative Commons licence, and indicate if changes were made. The images or other third party material in this article are included in the article's Creative Commons licence, unless indicated otherwise in a credit line to the material. If material is not included in the article's Creative Commons licence and your intended use is not permitted by statutory regulation or exceeds the permitted use, you will need to obtain permission directly from the copyright holder. To view a copy of this licence, visit http://creativecommons.org/licenses/by/4.0/.

\section{Appendix}

\section{A.1 Auxiliary Results}

Let $d=2$ and assume Hypothesis (H1). Suppose that $S: \mathbb{R}_{+} \times \mathbb{R}^{2} \rightarrow \mathbb{R}$ is a measurable function such that $S \in L^{2}\left(\mathbb{R}_{+} ; L^{2 q}\left(\mathbb{R}^{2}\right)\right)$, where $q$ is given in (2.20) in cases (a) and (b) and it is given in (2.23) in case (c). We assume also that $S$ has support in $[0, T] \times B_{M}$ for some $M>0$. We claim that $S$ belongs to $\mathcal{H}$ and the following estimates hold true:

$$
\|S\|_{\mathcal{H}} \leq \sqrt{\Gamma_{T}}\|S\|_{\mathcal{H}_{0}} \leq \sqrt{\Gamma_{T} D_{\gamma}}\|S\|_{L^{2}\left(\mathbb{R}_{+} ; L^{2 q}\left(\mathbb{R}^{2}\right)\right)}
$$

Indeed, the first inequality is due to (2.13) and the second one follows from (2.25).

For $d=1$, if $S \in L^{2}\left(\mathbb{R}_{+} \times \mathbb{R}\right)$ has support in $[0, T] \times B_{M}$ for some $M>0$, then $S \in \mathcal{H}$ and the following estimates hold true:

$$
\|S\|_{\mathcal{H}} \leq \sqrt{\Gamma_{T}}\|S\|_{\mathcal{H}_{0}} \leq \sqrt{\Gamma_{T}\left\|\gamma \mathbf{1}_{B_{2 M}}\right\|_{L^{1}(\mathbb{R})}}\|S\|_{L^{2}\left(\mathbb{R}_{+} \times \mathbb{R}\right)}
$$

Indeed, the first inequality is due to (2.13) and the second one follows from

$$
\begin{aligned}
\|S\|_{\mathcal{H}_{0}}^{2} & =\int_{0}^{T} \int_{\mathbb{R}^{2}} S(t, y) S\left(t, y^{\prime}\right) \gamma\left(y-y^{\prime}\right) d y d y^{\prime} d t \\
& \leq \int_{0}^{T} \int_{\mathbb{R}^{2}} \frac{S^{2}(t, y)+S^{2}\left(t, y^{\prime}\right)}{2} \gamma\left(y-y^{\prime}\right) d y d y^{\prime} d t
\end{aligned}
$$

and

$$
\sup _{y^{\prime} \in B_{M}} \int_{B_{M}} \gamma\left(y-y^{\prime}\right) d y \leq \int_{B_{2 M}} \gamma(y) d y .
$$

Let us recall the Hypothesis (H2): The measures $\mu_{0}$ and $\mu$ such that $\gamma_{0}=\mathcal{F} \mu_{0}$ and $\gamma=\mathcal{F} \mu$ are absolutely continuous with respect to the Lebesgue measures with strictly positive densities. 
Lemma A.1 Fix $d \in\{1,2\}$ and assume that the Hypothesis (H2) holds. Let the Hypothesis (H1) hold if in addition $d=2$. Suppose that the function $S: \mathbb{R}_{+} \times \mathbb{R}^{d} \rightarrow \mathbb{R}$ has support in $[0, T] \times B_{M}$ for some $M>0$ and $S \in L^{2}\left(\mathbb{R}_{+} ; L^{2 q}\left(\mathbb{R}^{d}\right)\right)$, where

$$
\left\{\begin{array}{l}
q \text { is given by (2.20) in cases (a) and (b) and by (2.23) in case (c) if } d=2, \\
q=1 \text { if } d=1 .
\end{array}\right.
$$

If

$$
I:=\int_{0}^{T} \int_{\mathbb{R}^{d}} \int_{\mathbb{R}^{d}} S(t, x) S(t, y) \gamma(x-y) d x d y d t>0
$$

then $\|S\|_{\mathcal{H}}>0$.

Proof Suppose that $\|S\|_{\mathcal{H}}=0$. There exists a sequence of smooth functions $\left(\psi_{k}\right)_{k \geq 1}$ in $C^{\infty}\left(\mathbb{R}_{+} \times \mathbb{R}^{d}\right)$, with support in $[0, T] \times B_{M}$, which converges to $S$ in $L^{2}\left(\mathbb{R}_{+} ; L^{2 q}\left(\mathbb{R}^{d}\right)\right)$. Then,

$$
0=\|S\|_{\mathcal{H}}^{2}=\lim _{k \rightarrow \infty}\left\|\psi_{k}\right\|_{\mathcal{H}}^{2}=\lim _{k \rightarrow \infty} \int_{\mathbb{R}_{+} \times \mathbb{R}^{d}}\left|\mathcal{F} \psi_{k}(\tau, \xi)\right|^{2} \mu_{0}(d \tau) \mu(d \xi),
$$

where $\gamma_{0}=\mathcal{F} \mu_{0}, \gamma=\mathcal{F} \mu$ and $\mathcal{F} \psi_{k}$ stands for the Fourier transform of $\psi_{k}$ in space-time variables in this proof. By choosing a subsequence $\left(k_{j}\right)_{j \geq 1}$ we have that

$$
\lim _{j \rightarrow \infty} \mathcal{F} \psi_{k_{j}}(\tau, \xi)=0
$$

for $\mu_{0} \otimes \mu$-almost all $(\tau, \xi)$. On the other hand, keeping in mind that the supports of $S, \psi_{k}$ are contained in $[0, T] \times B_{M}$, we have

$$
\begin{aligned}
\left\|\psi_{k}-S\right\|_{L^{1}\left(\mathbb{R}_{+} \times \mathbb{R}^{2}\right)} & \leq\left(\pi M^{2} T\right)^{1-\frac{1}{2 q}}\left\|\psi_{k}-S\right\|_{L^{2 q}\left(\mathbb{R}_{+} \times \mathbb{R}^{2}\right)} \\
& \leq\left(\pi M^{2}\right)^{1-\frac{1}{2 q}} T^{\frac{1}{2}}\left\|\psi_{k}-S\right\|_{L^{2}\left(\mathbb{R}_{+} ; L^{2 q}\left(\mathbb{R}^{2}\right)\right)},
\end{aligned}
$$

from which we deduce that $\left(\psi_{k}\right)_{k \geq 1}$ converge in $L^{1}\left([0, T] \times B_{M}\right)$ to $S$. Thus $\mathcal{F} \psi_{k}(\tau, \xi)$ converges to $\mathcal{F} S(\tau, \xi)$ for all $(\tau, \xi)$ and the convergence is uniform. As a consequence, $\mathcal{F} S(\tau, \xi)=0$ for $\mu_{0} \otimes \mu$-almost all $(\tau, \xi) \in \mathbb{R}_{+} \times \mathbb{R}^{d}$ and by Hypothesis (H2), we obtain $\mathcal{F} S(\tau, \xi)=0$ for almost all $(\tau, \xi) \in \mathbb{R}_{+} \times \mathbb{R}^{d}$ with respect to the Lebesgue measure.

Hence $S(t, x)=0$ for almost all $t>0$ and $x \in \mathbb{R}^{d}$, i.e. there exists a Borel set $N \subset \mathbb{R}_{+} \times \mathbb{R}^{d}$ with $\lambda_{d+1}(N)=0$ such that $S(t, x)=0$ for all $(t, x) \notin N$. Here $\lambda_{k}$ denotes the Lebesgue measure on $\mathbb{R}^{k}$. Therefore,

$$
I=\int_{0}^{\infty} \int_{\mathbb{R}^{d}} \int_{\mathbb{R}^{d}} 1_{A}(t, x, y) S(t, x) S(t, y) \gamma(x-y) d x d y d t,
$$

where $A:=\left\{(t, x, y) \in \mathbb{R}_{+} \times \mathbb{R}^{d} \times \mathbb{R}^{d} ;(t, x) \in N,(t, y) \in N\right\}$. 
Let $N_{t}=\left\{x \in \mathbb{R}^{d} ;(t, x) \in N\right\}$ be the section of the set $N$ at point $t>0$. By Fubini's theorem, $\lambda_{d+1}(N)=\int_{0}^{\infty} \lambda_{d}\left(N_{t}\right) d t$. Since $\lambda_{d+1}(N)=0$, we infer that $\lambda_{d}\left(N_{t}\right)=0$ for almost all $t>0$. Note that the section of the set $A$ at point $t$ is $A_{t}=\left\{(x, y) \in \mathbb{R}^{d} \times \mathbb{R}^{d} ;(t, x, y) \in A\right\}=N_{t} \times N_{t}$, and its Lebesque measure is $\lambda_{2 d}\left(A_{t}\right)=\lambda_{d}^{2}\left(N_{t}\right)=0$ for almost all $t>0$. By applying Fubini again, we infer that $\lambda_{2 d+1}(A)=\int_{0}^{\infty} \lambda_{2 d}\left(A_{t}\right) d t=0$. This shows $I=0$, which contradicts (A.1).

\section{A.2 Proof of Proposition 1.9}

In this section, we only sketch the proof of Proposition 1.9 as the main body of the proof is almost identical to that in [42, Proposition 3.2].

Proof of (1.27) Using the duality relation (2.5) and the identity $L=-\delta D$, we have

$$
\begin{aligned}
& \mathbb{E}\left[\left\langle D F,-D L^{-1} G\right\rangle_{\mathcal{H}}\right] \\
& \quad=\mathbb{E}\left[F(-\delta D) L^{-1} G\right]=\mathbb{E}\left[F L L^{-1} G\right]=\mathbb{E}[F G]=\operatorname{Cov}(F, G),
\end{aligned}
$$

which shows the equality in (1.27). Then, applying the Gaussian Poincaré inequality (2.12) and using Lemma 3.2 of [26], we can bound the variance appearing in the left-hand side of (1.27) by

$$
\begin{aligned}
\mathbb{E}\left[\left\|D\left\langle D F,-D L^{-1} G\right\rangle_{\mathcal{H}}\right\|_{\mathcal{H}}^{2}\right] \leq & 2 \mathbb{E}\left[\left\|\left\langle D^{2} F,-D L^{-1} G\right\rangle_{\mathcal{H}}\right\|_{\mathcal{H}}^{2}\right] \\
& +2 \mathbb{E}\left[\left\|\left\langle D F,-D^{2} L^{-1} G\right\rangle_{\mathcal{H}}\right\|_{\mathcal{H}}^{2}\right]
\end{aligned}
$$

We will show that the first expectation-term is bounded by $A_{1}$ and the other one can be estimated in the same way and bounded by $A_{2}$. Using the representation (see e.g. [25, Proposition 2.9.3])

$$
-D L^{-1} G=\int_{0}^{\infty} d t e^{-t} P_{t} D G
$$

with $\left\{P_{t}, t \geq 0\right\}$ the Ornstein-Uhlenbeck semigroup, we can write

$$
\left\langle D^{2} F,-D L^{-1} G\right\rangle_{\mathcal{H}}=\int_{0}^{\infty} d t e^{-t}\left\langle D^{2} F, P_{t} D G\right\rangle_{\mathcal{H}} .
$$

Note that if $(\mathcal{M}, \mathfrak{M}, v)$ is a probability space on which $s \in \mathcal{M} \longmapsto V_{s} \in|\mathcal{H}|$ is $\mathfrak{M}$-measurable such that $\int_{\mathcal{M}}\left\|\left|V_{s}\right|\right\|_{\mathcal{H}^{\nu}}^{2} v(d s)<\infty$, then by Fubini's theorem and Cauchy-Schwarz inequality,

$$
\begin{aligned}
\left\|\int_{\mathcal{M}} V_{s} v(d s)\right\|_{\mathcal{H}}^{2} & =\int_{\mathcal{M}^{2}}\left\langle V_{s}, V_{s^{\prime}}\right\rangle_{\mathcal{H}} v(d s) v\left(d s^{\prime}\right) \\
& \leq \int_{\mathcal{M}^{2}} \frac{\left\|V_{s}\right\|_{\mathcal{H}}^{2}+\left\|V_{s^{\prime}}\right\|_{\mathcal{H}}^{2}}{2} v(d s) v\left(d s^{\prime}\right)=\int_{\mathcal{M}}\left\|V_{s}\right\|_{\mathcal{H}}^{2} v(d s) .
\end{aligned}
$$


Using the above inequality on $\left(\mathbb{R}_{+}, e^{-t} d t\right)$, we deduce from (6.2) that

$$
\left\|\left\langle D^{2} F,-D L^{-1} G\right\rangle_{\mathcal{H}}\right\|_{\mathcal{H}}^{2} \leq \int_{0}^{\infty} d t e^{-t}\left\|\left\langle D^{2} F, P_{t} D G\right\rangle_{\mathcal{H}}\right\|_{\mathcal{H}}^{2}
$$

Observe that $\left\langle D^{2} F, P_{t} D G\right\rangle_{\mathcal{H}}$ is nothing else but the one-contraction $D^{2} F \otimes_{1} P_{t} D G$, so that

$$
\begin{aligned}
\left\|\left\langle D^{2} F, P_{t} D G\right\rangle_{\mathcal{H}}\right\|_{\mathcal{H}}^{2} & =\left\langle D^{2} F \otimes_{1} P_{t} D G, D^{2} F \otimes_{1} P_{t} D G\right\rangle_{\mathcal{H}} \\
& =\left\langle D^{2} F \otimes_{1} D^{2} F,\left(P_{t} D G\right) \otimes\left(P_{t} D G\right)\right\rangle_{\mathcal{H}^{\otimes 2}},
\end{aligned}
$$

where the last equality follows from the definition of contractions. Therefore, we have

$$
\begin{aligned}
\mathbb{E}[\| & \left.\left\langle D^{2} F,-D L^{-1} G\right\rangle_{\mathcal{H}} \|_{\mathcal{H}}^{2}\right] \\
\leq & \mathbb{E} \int_{0}^{\infty} d t e^{-t} \int_{\mathbb{R}_{+}^{6} \times \mathbb{R}^{6 d}} d r d r^{\prime} d s d s^{\prime} d \theta d \theta^{\prime} d z d z^{\prime} \\
& \times d y d y^{\prime} d w d w^{\prime} \gamma_{0}\left(\theta-\theta^{\prime}\right) \gamma_{0}\left(s-s^{\prime}\right) \gamma_{0}\left(r-r^{\prime}\right) \gamma\left(z-z^{\prime}\right) \gamma\left(w-w^{\prime}\right) \gamma\left(y-y^{\prime}\right) \\
& \times\left[D_{r, z} D_{\theta, w} F\right]\left[D_{s, y} D_{\theta^{\prime}, w^{\prime}} F\right] P_{t}\left(D_{r^{\prime}, z^{\prime}} G\right) P_{t}\left(D_{s^{\prime}, y^{\prime}} G\right)
\end{aligned}
$$

and thus we end our estimation of $\mathbb{E}\left[\left\|\left\langle D^{2} F,-D L^{-1} G\right\rangle_{\mathcal{H}}\right\|_{\mathcal{H}}^{2}\right]$ by using Hölder inequality and the contraction property of $P_{t}$ on $L^{4}(\Omega)$, that is, using $\left\|P_{t}\left(D_{r^{\prime}, z^{\prime}} G\right)\right\|_{4} \leq$ $\left\|D_{r^{\prime}, z^{\prime}} G\right\|_{4}$.

To estimate the other expectation-term $\mathbb{E}\left[\left\|\left\langle D F,-D^{2} L^{-1} G\right\rangle_{\mathcal{H}}\right\|_{\mathcal{H}}^{2}\right]$, one can begin with

$$
-D^{2} L^{-1} G=\int_{0}^{\infty} d t e^{-2 t} P_{t} D^{2} G
$$

and then follow the same arguments.

\section{References}

1. Balan, R.M.: The stochastic wave equation with multiplicative fractional noise: a Malliavin calculus approach. Potential Anal. 36, 1-34 (2012)

2. Balan, R.M., Quer-Sardanyons, L., Song, J.: Existence of density for the stochastic wave equation with space-time homogeneous Gaussian noise. Electron. J. Probab. 24(106), 1-43 (2019)

3. Balan, R.M., Song, J.: Hyperbolic Anderson Model with space-time homogeneous Gaussian noise. ALEA Lat. Am. J. Probab. Math. Stat. 14, 799-849 (2017)

4. Bolaños-Guerrero, R., Nualart, D., Zheng, G.: Averaging 2D stochastic wave equation. Electron. J. Probab. 26(102), 1-32 (2021)

5. Bouleau N., Hirsch, F.: Propriété d'absolue continuité dans les espaces de Dirichlet et applications aux équations différentielles stochastiques. Séminaire de Probabilités XX: 12, 131-161, LNM 1204 (1986)

6. Breuer, P., Major, P.: Central limit theorems for non-linear functionals of Gaussian fields. J. Multivar. Anal. 13, 425-441 (1983) 
7. Carmona, R., Nualart, D.: Random non-linear wave equations: smoothness of the solutions. Probab. Theory Related Fields 79, 469-508 (1988)

8. Chatterjee, C.: Fluctuation of eigenvalues and second order Poincaré inequalities. Probab. Theory Related Fields 143, 1-40 (2009)

9. Chen L., Khoshnevisan D., Nualart, D., Pu, F.: Poincaré inequality, and central limit theorems for parabolic stochastic partial differential equations. To appear in: Ann. Inst. Henri Poincaré Probab. Stat. (2021) arXiv:1912.01482

10. Chen L., Khoshnevisan D., Nualart, D., Pu, F.: Central limit theorems for spatial averages of the stochastic heat equation via Malliavin-Stein's method. To appear in Stoch. Partial Differ. Equ. Anal. Comput. (2020). arXiv:2008.02408

11. Dalang, R.C.: Extending the martingale measure stochastic integral with applications to spatially homogeneous. Electron. J. Probab. 4(6), 1-29 (1999)

12. Delgado-Vences, F., Nualart, D., Zheng, G.: A central limit theorem for the stochastic wave equation with fractional noise. Ann. Inst. Henri Poincaré Probab. Stat. 56(4), 3020-3042 (2020)

13. Dunlap, A., Gu, Y., Ryzhik, L., Zeitouni, O.: Fluctuations of the solutions to the KPZ equation in dimensions three and higher, p. 176. Probab, Theory Related Fields (2020)

14. Houdré, C., Pérez-Abreu, V.: Covariance identities and inequalities for functionals on Wiener and Poisson spaces. Ann. Probab. 23, 400-419 (1995)

15. Huang, J., Nualart, D., Viitasaari, L.: A central limit theorem for the stochastic heat equation. Stoch. Process. Appl. 130(12), 7170-7184 (2020)

16. Huang, J., Nualart, D., Viitasaari, L., Zheng, G.: Gaussian fluctuations for the stochastic heat equation with colored noise. Stoch. Partial Differ. Equ. Anal. Comput. 8, 402-421 (2020)

17. Kallenberg, O.: Foundations of Modern Probability, Probability and Its Applications, 2nd edn. Springer, New York (2002)

18. Karkzewska, A., Zabczyk, J.: Stochastic PDE's with function-valued solutions. In: Infinite-dimensional stochastic analysis (Clément Ph., den Hollander F., van Neerven J. \& de Pagter B., eds), pp. 197-216, Proceedings of the Colloquium of the Royal Netherlands Academy of Arts and Sciences, Amsterdam (1999)

19. Khoshnevisan, D., Nualart, D., Pu, F.: Spatial stationarity, ergodicity and CLT for parabolic Anderson model with delta initial condition in dimension $d \geq 1$. SIAM J. Math. Anal. 53(2), 2084-2133 (2021)

20. Kim, K., Yi, J.: Limit theorems for time-dependent averages of nonlinear stochastic heat equations. To appear in: Bernoulli. (2021+). arXiv:2009.09658

21. Malliavin, P.: Stochastic calculus of variations and hypoelliptic operators. Proceedings of the International Symposium on Stochastic Differential Equations (Res. Inst. Math. Sci., Kyoto Univ., Kyoto, 1976). New York: Wiley. pp. 195-263 (1978)

22. Márquez-Carreras, D., Mellouk, M., Sarrà, M.: On stochastic partial differential equations with spatially correlated noise: smoothness of the law. Stoch. Process. Appl. 93, 269-284 (2001)

23. Millet, A., Sanz-Solé, M.: A stochastic wave equation in two space dimension: smoothness of the law. Ann. Probab. 27, 803-844 (1999)

24. Nourdin, I., Peccati, G.: Stein's method on Wiener chaos. Probab. Theory Related Fields 145(1), 75-118 (2009)

25. Nourdin, I., Peccati, G.: Normal approximations with Malliavin calculus: from Stein's method to universality. Cambridge Tracts in Mathematics 192, Cambridge University Press (2012)

26. Nourdin, I., Peccati, G., Reinert, G.: Second order Poincaré inequalities and CLTs on Wiener space. J. Funct. Anal. 257, 593-609 (2009)

27. Nualart, D.: The Malliavin Calculus and Related Topics, Probability and Its Applications, 2nd edn. Springer, Berlin (2006)

28. Nualart, D., Ortiz-Latorre, S.: Central limit theorems for multiple stochastic integrals and Malliavin calculus. Stoch. Process. Appl. 118(4), 614-628 (2008)

29. Nualart, D., Pardoux, É.: Stochastic calculus with anticipating integrands. Probab. Theory Related Fields 78, 535-581 (1988)

30. Nualart, D., Peccati, G.: Central limit theorems for sequences of multiple stochastic integrals. Ann. Probab. 33(1), 177-193 (2005)

31. Nualart, D., Quer-Sardanyons, L.: Existence and smoothness of the density for spatially homogeneous SPDEs. Potential Anal. 27, 281-299 (2007)

32. Nualart, D., Song, X.M., Zheng, G.: Spatial averages for the Parabolic Anderson model driven by rough noise. ALEA Lat. Am. J. Probab. Math. Stat. 18, 907-943 (2021) 
33. Nualart, D., Zheng, G.: Averaging Gaussian functionals. Electron. J. Probab. 25(48), 1-54 (2020)

34. Nualart, D., Xia, P., Zheng, G.: Quantitative central limit theorems for the parabolic Anderson model driven by colored noises. (2021) arXiv:2109.03875

35. Nualart, D., Zheng, G.: Central limit theorems for stochastic wave equations in dimensions one and two. To appear in Stoch. Partial Differ. Equ. Anal. Comput (2021)

36. Nualart, D., Zheng, G.: Spatial ergodicity of stochastic wave equations in dimensions 1,2 and 3. Electron. Commun. Probab. 25(80), 1-11 (2020)

37. Peccati, G., Tudor, C.A.: Gaussian limits for vector-valued multiple stochastic integrals. Séminaire de Probabilités XXXVIII, 247-262 (2005)

38. $\mathrm{Pu}, \mathrm{F}$ : Gaussian fluctuation for spatial average of parabolic Anderson model with Neumann/Dirichlet/periodic boundary conditions. Trans. Amer. Math. Soc. (2021). https://doi.org/10. $1090 / \operatorname{tran} / 8565$

39. Quer-Sardanyons, L., Sanz-Solé, M.: Absolute continuity of the law of the solution to the 3-dimensional stochastic wave equation. J. Funct. Anal. 206(1), 1-32 (2004)

40. Quer-Sardanyons, L., Sanz-Solé, M.: A stochastic wave equation in dimension 3: Smoothness of the law. Bernoulli 10(1), 165-186 (2004)

41. Sanz-Solé, M., Süss, A.: The stochastic wave equation in high dimensions: Malliavin differentiability and absolute continuity. Electron. J. Probab. 18(64), 1-28 (2013)

42. Vidotto, A.: An improved second-order Poincaré inequality for functionals of Gaussian fields. J. Theoret. Probab. 33, 396-427 (2020)

43. Walsh, J.B.: An Introduction to Stochastic Partial Differential Equations. In: École d'été de probabilités de Saint-Flour, XIV—1984, 265-439. Lecture Notes in Math. 1180, Springer, Berlin (1986)

44. Zheng, G.: Recent developments around the Malliavin-Stein approach-fourth moment phenomena via exchangeable pairs. Ph.D thesis, Université du Luxembourg. (2018) Available at http://hdl.handle. net/10993/35536

Publisher's Note Springer Nature remains neutral with regard to jurisdictional claims in published maps and institutional affiliations.

\section{Authors and Affiliations}

\section{Raluca M. Balan ${ }^{1}$ - David Nualart ${ }^{2}$ - Lluís Quer-Sardanyons ${ }^{3}$. Guangqu Zheng ${ }^{4}$}

Raluca M. Balan

rbalan@uottawa.ca

David Nualart

nualart@ku.edu

Lluís Quer-Sardanyons

quer@mat.uab.cat

1 Department of Mathematics and Statistics, STEM Building, University of Ottawa, 150

Louis-Pasteur Private, Ottawa, ON K1N 6N5, Canada

2 Department of Mathematics, University of Kansas, 405 Snow Hall, Lawrence, KS 66045, USA

3 Departament de Matemàtiques, Universitat Autònoma de Barcelona, 08193, Cerdanyola del Vallès Catalonia, Spain

4 School of Mathematics, The University of Edinburgh, James Clerk Maxwell Building, Peter Guthrie Tait Road, Edinburgh EH9 3FD, UK 